LBL -3.1970

DE92 017025

\title{
Modelling of 3-D Electromagnetic Responses Using the Time-Wavenumber Method
}

\author{
Seunghee Lee \\ (Ph.D. Thesis) \\ Engineering Geoscience \\ University of California \\ and \\ Earth Sciences Division \\ Lawrence Berkeley Laboratory \\ University of California \\ Berkeley, California 94720
}

December 1991

This work was supported by the Director, Orfice of Energy Research, Office of Basic Energy Sciences, Engineering and Geosciences Division, and by the Assistant Secretary for Fossil Energy, Office of Oil, Gas and Shale Technologies, of the U.S. Deparment of Energy under Contract No. DE-AC03-76SFCI098. 


\begin{abstract}
The diffusion of electromagnetic fields in time and the three spatial dimensions can be modelled using a new numerical algorithm that is tailored for geophysical applications. The novel feature of the algorithm is that a large part of the computation is done in the wavenumber domain. Here, the spatial Fourier transforms of the vertical magnetic field and the vertical current density are used to define two scalar potentials. For either a vertical electric or a vertical magnetic dipole source at the subsurface these wavenumber potentials can be represented by a simple Gaussian distribution function. In the air, the fields satisfy the Laplace equation.

The flow of this algorithm is as follows: the potentials are defined in the wavenumber domain as an initial condition depending on the source configuration, the vector current density $J$ in space is obtained from the potentials using the inverse Fourier transform, the vector electric field $\mathrm{E}$ is obtained by multiplying $\mathrm{J}$ by resistivity, the updated potentials are then obtained from the forward Fourier transform of $E$. Using the updated potential as a subsequent initial condition these steps are repeated until the solution reaches the final tirne.

Since spatial derivatives can be exactly evaluated in the wavenumber domain by simple multiplications, this algorithm requires far less memory than the conventional finite difference (FD) method. The conventional FD method needs finer discretization in space in order to minimize the numerical dispersion caused by numerical differentiation in space. The conductivity distribution for this algorithm is piece-wise continuous and bounded in the wavenumber domain. A model of $64 \times 64 \times 32$ nodes requires one megaword of storage and 50 giga floating point operations for 3 decades in time. This computation is equivalent to one half CPU hour on a Cray"? with 1 parallel processor withour any serious optimization of the program.

A realistic 3-D model still requires millions of words of computational memory and giga flops in order 10 proceed to a few decades in time. The simple algorithm presented here can be highly vectorized and is then ideally suited for modern parallel computers.
\end{abstract}

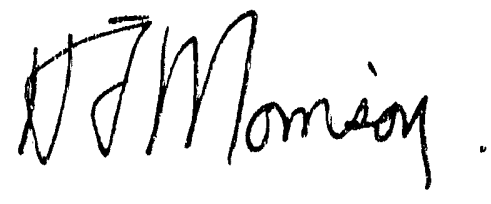




\section{ACKNOWLEDGMENT}

I am grateful to my supervising professor H. F. Morrison for guidance to finish this work, my advisor Prof. A. Becker for his constructive comments, and my dissertation committee Prof. K. K. Mei for reading the manuscript. I am also thankful to Prof. G. Egbert, Dr. Kiha Lee and all the graduate students in Engineering Geoscience for constructive discussions.

This work was supported by Office of Basic Energy Sciences and Office of Oil, Gas, and Shale Technology, of the U. S. Department of Energy under contract no. DE-ACO376 SF00098. 


\section{TABLE OF CONTENTS}

Chapter $1 \quad$ Introduction.................................................. 1

Chapter 2 Theory.............................................................. 5

2.1 Maxwell's equations in the $t-k$ domain.................................. 5

2.2 The air-earth interface............................................ 9

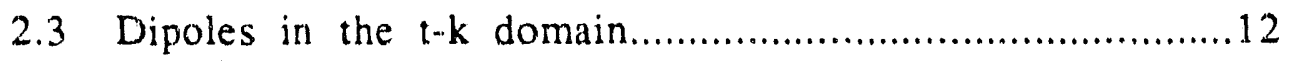

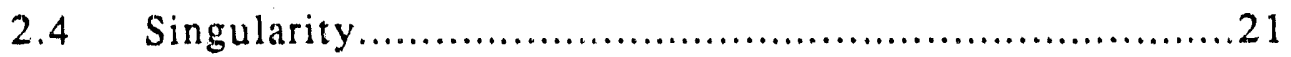

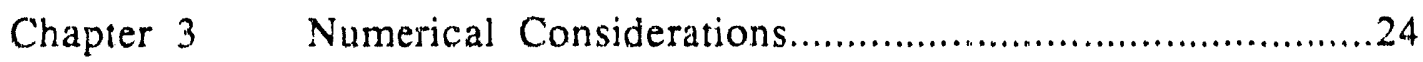

3.1 Modified forward time-stepping........................................24

3.2 Numerical boundary and grid-spacing..................................31

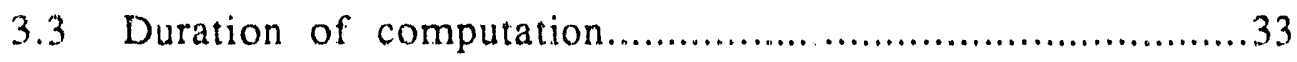

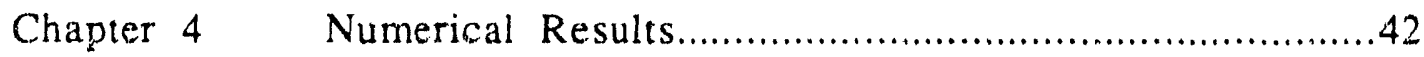

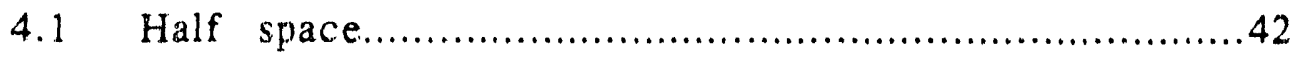

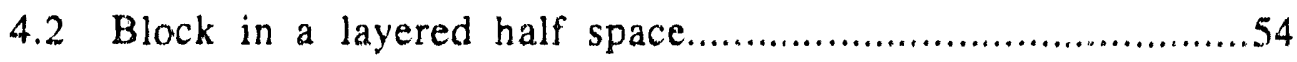

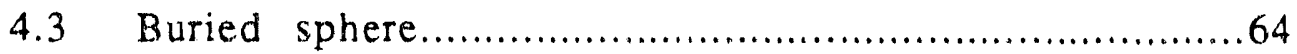

Chapter 5 Discussion, Useful Derivations and Summary..........................70

5.1 Direct convolution................................................... 71

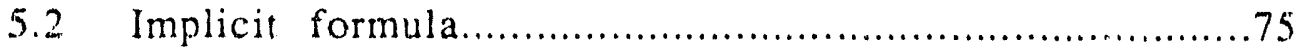

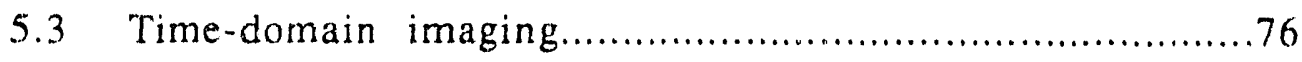

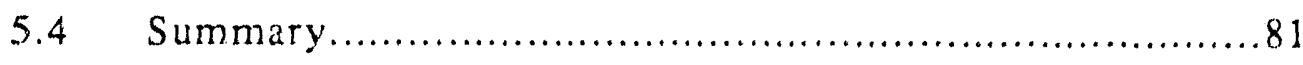

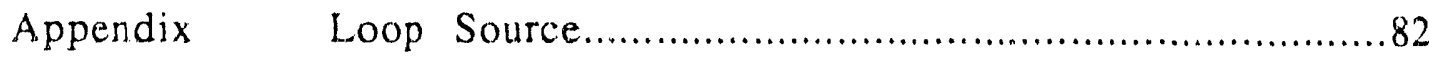

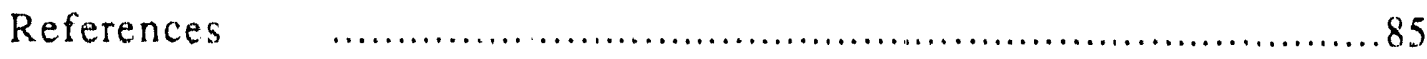




\section{TABLE OF ILLUSTRATIONS}

Figure 2-1 Flow chart of the $\mathrm{t}-\mathrm{k}$ algorithm.

Figure 2-2 Fourier transform pairs for real, symmetric, and causal functions.

Figure 2-3 Schematic diagram of vertical magnetic and electric dipole fields.

Figure 2-4 Horizontal current density $\left(\mathrm{J}_{\mathrm{y}}\right)$ for a step current magnetic dipole (the dipole is at the center of 10 Ohm.m full space, 1 and 10 usec snapshots).

Figure 2-5 Horizontal current density $\left(\mathrm{J}_{\mathrm{X}}\right)$ for an impulse current electric dipole the dipole is at the center of 10 Ohm.m full space, 1 and 10 usec snapshots).

Figure 2-6 Vertical current density $\left(\mathrm{J}_{\mathrm{Z}}\right)$ for an impulse current electric dipole (the dipole is at the center of 10 Ohm.m full space, 1 and 10 usec snapshots).

Figure 2-7 Scalar potential f for a step current magnetic dipole.

Figure 2-8 Scalar potential g for an impulse current electric dipole.

Figure 2-9 Transformation of a surface integral to a line integral using Stoke's theorem.

Figure 3-1 Equivalence principle (after Harrington, 1963).

Figure 3.2 Relative error in the spectral amplitude of the potential $f$ and $g$ as a function of finite grid-spacing.

Figure 3-3 (a) Grid-spacing and numerical boundary for each decade. A vertical magnetic dipole is at the center of grid. Each volume contains $64 \times 64 \times 32$ nodes. 
Figure 3-3 (b) Sn:pshots of the tangential current density $\left(\mathrm{J}_{\mathrm{y}}\right)$ at the starting and ending time ( 1 and 10 usec) of computation at the first grid configuration. The minor asymmetry in the figure is an artifact of the plotting software.

Figure 3-3 (c) Snapshots of $\mathrm{J}_{\mathrm{y}}$ at 10 and 100 usec at 2 nd decade.

Figure 3-3 (d) Snapshots of $\mathrm{J}_{\mathrm{y}}$ at 100 and 1000 usec at 3rd decade.

Figure 3-4 Scalar Green's function (diffusion equation) $100 \mathrm{~m}$ away from the source. Arrows indicate the arrival time of wavefront (in the case of wave equation) when $\varepsilon=1,9,80 \varepsilon_{0}$ respectively.

Figure 3-5 Illustration of the latest possible starting time and earliest possible ending time in the numerical calculation.

Figure 4-1 Whole and half space model. The source is at the center for the whole space and $25 \mathrm{~m}$ below surface for the half space.

Figure 4-2 (a)Snapshots of the tangential electric field (Ey) $(t=0.5$ usec).

Figure 4-2 (b)Snapshots of Ey ( $t=5$. usec).

Figure 4-2 (c) Snapshots of Ey( $t=10$. usec).

Figure 4-2 (d)Snapshots of $E y(t=50$. usec).

Figure 4-3 Time traces of Ey at the surface. Each trace has shifted by $2 u \mathrm{~V} / \mathrm{m}$ (linear amplitude and time scales).

Figure 4-4 (a)Comparisons with 1-D pseudo-analytic solution at $(30,0,0)$ and $(30,0,-5)$.

Figure 4-4 (b)Comparisons with 1-D pseudo-analytic solution at $(50,0,0)$ and $(50,0,25)$.

Figure 4-5 3-D model. A cube $(10 \times 10 \times 10 \mathrm{~m})$ is at $40 \mathrm{~m}$ below surface and $15 \mathrm{~m}$ away from the source. The righthand side diagram show's the resistivity of the model layers. 
Figure 4-6 (a) Snapshots of Ey at .1 and 1 usec.

Figure 4-6 (b) Snapshots of Ey at 3 and 10 usec.

Figure 4-7 Time traces of Ey at the vertical observation hole with or without the block. Each trace has shifted by $10 \mathrm{uV} / \mathrm{m}$ (linear amplitude and time scales).

Figure 4-8 Time traces of vertical magnetic field at the vertical observation hole with or without the block. Each trace has shifted by $1 \mathrm{~A} / \mathrm{m} / \mathrm{s}$ (linear amplitude and time scales).

Figure 4-9 Comparisons of the tangential electric field for the layer model with the 1-D pseudo-analytic solution along the observation hole .

Figure 4-10 Comparisons of the vertical magnetic field for the layer model with the 1-D pseudo-analytic solution $(-30,0,40)$.

Figure 4-11 Comparisons of the vertical magnetic field for the 3-D model with the 3-D integral equation solution $(-30,0,40)$ (2ndary $\mathrm{H}_{\mathrm{z}}=3-\mathrm{D} \mathrm{H}-1-\mathrm{D} \mathrm{H}$ ).

Figure 4-12 Buried sphere model and the snapshot of Ey at an early time (1 usec).

Figure 4-13 (a) Snapshots of half space and with sphere model at $t=5 \mathrm{usec}$ (Ey).

Figure 4-13 (b) Snapshots of half space and with sphere model at $t=10 \mathrm{usec}$ (Ey).

Figure 4-13 (c) Snapshots of half space and with sphere model at $t=50$ usec (Ey).

Figure 4-14 Comparisons with the sphere solution (Lee, 1976). The pseudo-anaiytic sphere solution is evaluated only in late times.

Figure 5-1 Examples of Fourier transform pair $(z<->k z)$.

Figure 5-2 Results of double-precision (64 bit) floating-point FFT (N=32). The numerical precision range is $9 \sim 10$ decimal digits as shown in these figures. 
Figure 5-3 Schematic diagram of a realistic 3-D model and the area where one can use a 1D or a $2-D$ solution at early tirnes.

Figure 5-4 Primary and secondary conductivity distributions. Both distributions can be arbitrary but excluding small area near the source (limi $->0)$ for the secondary distribution. 


\section{Introduction}

The diffusion of electromagnetic fields in conducting media is of great practical importance in geophysical exploration for subsurface targets such as ore deposits, hydrocarbon accumulations, geothermal reservoirs, groundwater or contamination plumes. The major difficulty in these exploration and exploitation task s is the lack of effective interpretational aids. Although modern field systems can collect data in a wide variety of configurations with an accuracy unheard of a decade ago, the tools available to interpret these data are still at a primitive level.

Like many other physical properties measured with geophysical methods, the subsurface electrical conductivity distribution is very complex and mostly random. Analytic solutions for the response of various measurement configurations are available only for one dimensional layered distributions and for very simple inhomogeneous models such as buried spheres or cylinders.

For modelling two dimensional conductivity distributions, the vector fields of Maxwell's equations can be decoupled into one or two scalar equations. Numerical methods based on the approximation of differentials by finite differences, finite element schemes which minimize the energy stored in the system, and integral equations which solve for unknown fields inside confined bodies implanted in the known primary fields, or some combination of these methods, have been successfully implemented and published in many geophysical journals in the past decades (e.g. Jones and Price, 1969; Coggon, 1971; Hohmann, 1971; Swift, 1971; Weaver and Brewitt-Taylor, 1978; Kuo and Cho, 1980; Hermance, 1982; Goldman and Stoyer, 1983). 
For the three dimensional case, the full vector diffusion equation must be solved. Most attempts have been formulated in the frequency domain. Again, a range of numerical techniques has been applied to this problem including the finite difference method (e.g. Line and Jones, 1973; Zhadanov et. al., 1982), the finite element method (e.g. Reddy et. al., 1977; Pridmore et. al., 1981), the integral equation method (e.g. Raiche, 1974; Hohmann, 1975; Weidelt, 1975), and the hybrid method which attempts to combine advantages from the finite element and integral equation methods (e.g. Lee et. al., 1981; Gupta et. al., 1987).

Finite differsace or finite element methods, which are suitable for modelling realistic 3 dimensional complex geometry, have been attempted only for sparsely grided simple bodies since they require huge memory and a large number of calculations. The integral equation methods, which require less computational memory and fewer calculations, can only be applied to confined bodies in a layered earth.

Time domain solutions for electromagnetic problems can be obtained by a Fourier transform of frequency domain results. However, not only does this approach require a large number of frequency domain results, but existing frequency domain solutions generally tend to be unstable at high frequencies (Newmann et. al., 1986). A direct time domain solution using the integral equation method was obtained by San Filipo et. al. (1985). But this method is still restricted to confined bodies in a layered earth.

A direct time domain solution (without the diffusion approximation) using a finite difference method was introduced by Yee (1966). This method solves for the electric and magnetic vector fields via a time-stepping algo- 
rithm. The electric and magnetic fields are defined at different nodes (staggered grid) and solved at alternate time steps (leap-frog time-stepping). Although this method has been adapted to some three dimensional "geophysical type" electromagnetic problems (Tayler et. al., 1969; Kunz and Lee, 1978), application to realistic geophysical problems is problematic. The fields have to propagate with the displacement current (usually negligible in the actual geophysical situations) and this method then requires a large number of small time steps to solve the equation for the time range of interest in geophysical situations.

A fundamental aspect of the finite difference and finite element methods is that the grid is established to provide numerical approximations for the derivatives that must be evaluated. A distinctive feature of the algorithm developed here is the evaluation of spatial derivatives in the wavenumber domain. The evaluation of spatial derivatives in the wavenumber domain by simple multiplication has been previously applied to two dimensional geophysical acoustic (Gazdag, 1981; Kosloff and Baysal, 1982) and elastic (Kosloff et. al., 1984) wave propagation problems (pseudo-spectral method). In these seismic exercises, it was shown that this method requires less nodes than other finite difference or finite element methods, an advantage which is crucial for large three dimensional grids.

In the next chapter, the vector diffusion equation will be decoupled into two coupled scalar diffusion equations for the time-stepping algorithm. Initial conditions and the air-earth interface are treated. In chapter 3 , new concepts of time-stepping and grid-spacing based on the exponentially decaying diffusion process in the wavenumber domain will be implemented. In chapter 
4, various numerical steps toward the solution will be examined and the results will be compared with other available solutions. In the last chapter, limitations of this algorithm for modelling and useful derivations from the invariant, linear, and harmonic nature of diffusion process will be discussed. 


\section{Theory}

In this chapter, Maxwell's equations will be forinulated in the timewavenumber ( $i-k$ ) domain for a time-stepping algorithm. The air-earth interface, where the diffusion velocity goes to infinity and the time-stepping algorithm becomes unstable, will be treated analytically. The initial condition, an electric or magnetic dipole in the whole space, will be derived in the $\mathrm{t}-\mathrm{k}$ domain to show the harmonic aspect of the diffusion process.

\subsection{Maxwell's equations in $t-k$ domain}

Maxwell's equations, neglecting the displacement current $\left(\epsilon \frac{\partial E}{\partial t} \ll \sigma \vec{E}\right.$ ) and assuming that the magnetic permeability is constant and equal to that of free space $\left(\mu_{0}=4 \pi \times 10^{-7} \mathrm{H} / \mathrm{m}\right)$, may be written as follows:

$$
\begin{gathered}
\nabla \cdot \vec{H}(x, y, z, t)=0, \\
\nabla \times \vec{H}(x, y, z, t)=\vec{J}(x, y, z, t), \\
\nabla \times \vec{E}(x, y, z, t)=-\mu_{0} \frac{\partial}{\partial t} \vec{H}(x, y, z, t), \\
\nabla \times \nabla \times \vec{E}(x, y, z, t)=-\mu_{0} \frac{\partial}{\partial t} \vec{J}(x, y, z, t) .
\end{gathered}
$$

If the resistivity distribution is piece.w.se continuous, then

$$
\vec{E}(x, y, z, t)=\rho(x, y, z) \vec{J}(x, y, z, t)
$$

$\vec{E}$ and $\vec{H}$ are the electric and magnetic vector fields respectively, $\vec{J}$ is the electric current density, and $\rho$ and $\mu_{0}$ are the resistivity and magnetic per. meability respectively, 
In the wavenumber domain, equations (2.1) and (2.2) becomes

$$
\begin{gathered}
k_{x} \tilde{H}_{x}+k_{y} \tilde{H}_{y}+k_{z} \tilde{H}_{z}=0 \\
i k_{y} \tilde{H}_{z}-i k_{z} \tilde{H}_{y}=\tilde{J}_{x} \\
i k_{z} \tilde{H}_{x}-i k_{x} \tilde{H}_{z}=\tilde{J}_{y} \\
i k_{x} \tilde{H}_{y}-i k_{y} \tilde{H}_{x}=\tilde{J}_{z} .
\end{gathered}
$$

where represents Fourier transform from the space $(x, y, z)$ to wavenumber $\left(k_{x}, k_{1}, k_{z}\right)$ domain and $i=\sqrt{-1}$.

In equation (2.6), we have 6 unknowns in 4 linear equations. Therefore, one can solve for any combination of 2 unknowns from the others. Solving for $\tilde{J}_{x}$ and $\tilde{J}_{y}$,

$$
\begin{gathered}
\tilde{J}_{x}=\frac{i k_{y}\left(k_{x}^{2}+k_{y}^{2}+k_{z}^{2}\right) \tilde{H}_{z}-k_{x} k_{z} j_{z}}{k_{x}^{2}+k_{y}^{2}} \\
\tilde{J}_{y}=\frac{-i k_{x}\left(k_{x}^{2}+k_{y}^{2}+k_{z}^{2}\right) \tilde{H}_{z}-k_{y} k_{x} \tilde{J}_{z}}{k_{x}^{2}+k_{y}^{2}}
\end{gathered}
$$

Equation (2.3) and (2.4) can then be used to express the time derivatives of $\tilde{H}_{z}$ and $\tilde{J}_{z}$ as

$$
\begin{gathered}
\mu_{0} \frac{\partial \tilde{H}_{z}}{\partial t}=i k_{y} \dot{E}_{x}-i k_{x} \tilde{E}_{y}, \\
\mu_{0} \frac{\partial \tilde{J}_{z}}{\partial t}=k_{x} k_{z} \tilde{E}_{x}+k_{y} k_{z} \tilde{E}_{y}-\left(k_{x}^{2}+k_{y}^{2}\right) \tilde{E}_{z} .
\end{gathered}
$$

Defining 2 scalar potentials as

$$
\begin{gathered}
f\left(k_{x}, k_{y}, k_{z}, t\right)=i \frac{\left(k_{x}^{2}+k_{y}^{2}+k_{z}^{2}\right) \dot{H}_{2}}{k_{x}^{2}+k_{y}^{2}}, \\
g\left(k_{x}, k_{y}, k_{z}, t\right)=\frac{k_{z} \tilde{J}_{z}}{k_{x}^{2}+k_{y}^{2}}
\end{gathered}
$$


By the definition $f$ and $g$, eqution (2.7) and (2.8) can be rewritten as

$$
\begin{gathered}
\tilde{J}_{x}=k_{y} f-k_{x} g, \\
\tilde{J}_{y}=-k_{x} f-k_{y} g, \\
\tilde{J}_{z}=\frac{k_{x}^{2}+k_{y}^{2}}{k_{z}} g .
\end{gathered}
$$

With these definitions and equations,

$$
\begin{gathered}
\mu_{0} \frac{\partial f}{\partial t}=\frac{k_{x}^{2}+k_{y}^{2}+k_{z}^{2}}{k_{x}^{2}+k_{y}^{2}}\left(k_{x} \tilde{E}_{y}-k_{y} \tilde{E}_{x}\right), \\
\mu_{0} \frac{\partial g}{\partial t}=\frac{k_{z}^{2}}{k_{x}^{2}+k_{y}^{2}}\left(k_{x} \tilde{E}_{x}+k_{y} \tilde{E}_{y}\right)-k_{z} \tilde{E}_{z} .
\end{gathered}
$$

We can declare the scalar potentials $f$ and $g$ as 2 orthogonal current density potentials since they have the same units as the magnetic field $[\mathrm{A} / \mathrm{m}]$ and the current densities can be derived from a linear combination of their spatial derivatives.

The governing diffusion equation can be solved as follows:

1) At some early time t, $H_{z}$ and $J_{z}$ and hence the scalar potentials $f$ and $g$ are known in the wavenumber domain.

2) The current densities $\tilde{J}_{x}, \tilde{J}_{y}$, and $\tilde{J}_{z}$ are obtained from $f$ and $g$ by equation (2.13), (2.14), and (2.15).

3) Transform these current densities from the wavenmber to space domain by the inverse Fourier transform.

4) The electric fields $E_{x}, E_{y}$, and $E_{z}$ are obtained by multiplying the curient densities by resistivity $\rho$.

5) Transform the electric fields from the space to wavenumber domain by the forward Fourier transfor in. 


\section{3-D EM Modeling Using t-k Method}

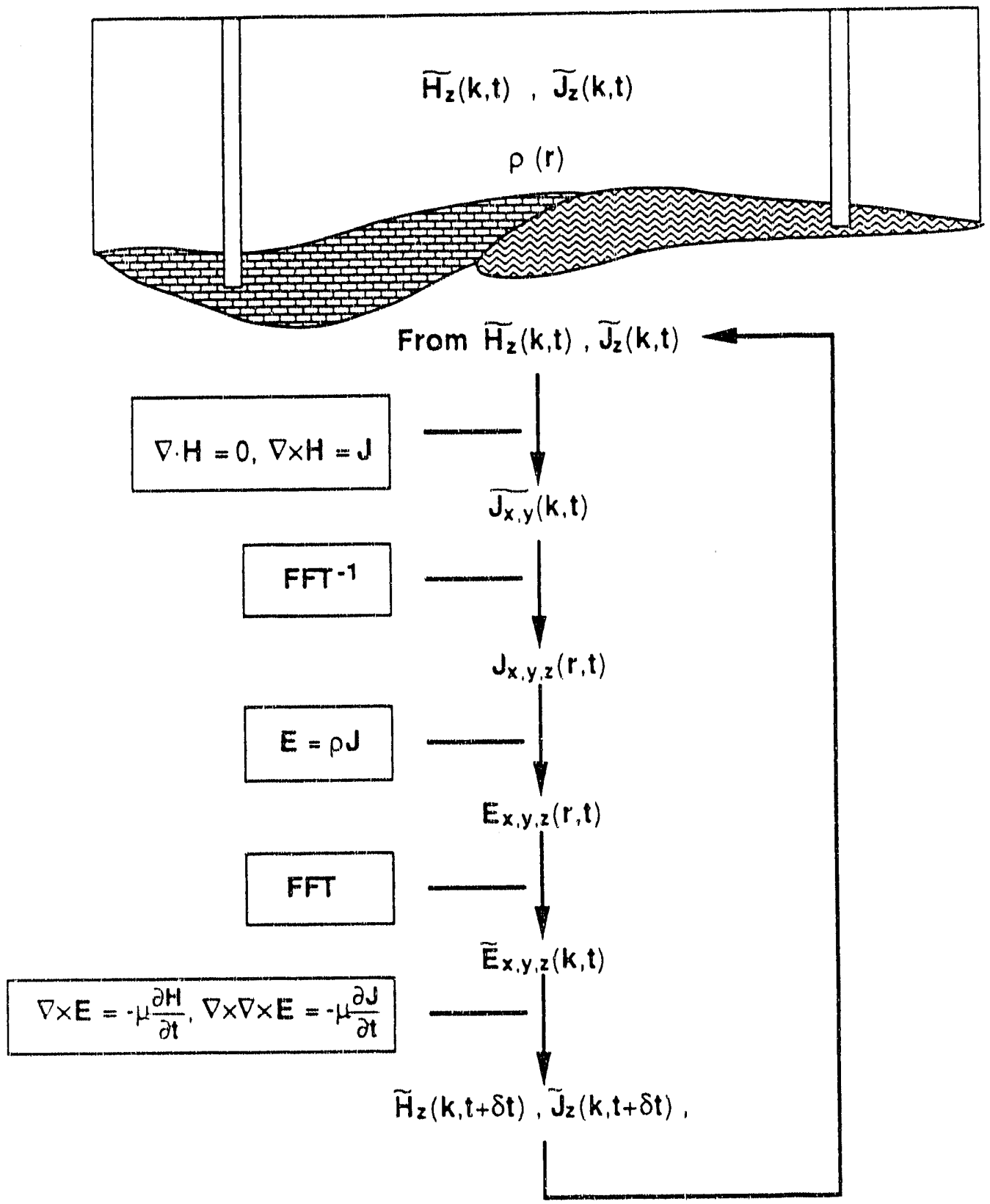

Figure 2-1 Flow chart of the $\mathrm{t}-\mathrm{k}$ algorithm. 
6) Using a proper time differencing formula, the values of $f$ and $g$ at the next time step are obtained from equation (2.16) and (2.17).

7) These new values of $f$ and $g$ are then inserted in step 1 and the above procedure is repeated until the solution reaches the final time.

Figure (2.1) shows the flow of this algorithm.

\subsection{Air-earth interface}

In a non-conducting medium $(\rho=\infty)$, the electric field exists but it can not be evaluated from the current density since the current density vanishes. Localized non-conducting bodies in the half space can be approximated by using resistivities much higher than the surrounding medium. The free space above the conducting half space can be treated analytically.

In free space, by definition,

$$
\begin{gathered}
\rho(x, y, z<0)=\infty \\
J_{x, y, z}(x, y, z<0)=0 .
\end{gathered}
$$

The continuity of the normal current further restricts the $z$ - component as

$$
J_{z}(x, y, z=0) \equiv 0
$$

The continuous resistivity distribution ensures that $J_{x, y, z}$ and $E_{x, y, z}$ are continuous in the subsurface. At the air-earth interface, the tangential electric fields $E_{x, y}$ are continuous, but the horizontal current densities $J_{x, y}$ are not. This discontinuity can be treated analytically using the causality of Fourier transform pairs. 
In free space, the vertical magnetic field satisfies Laplace's equation ( if $\left.\epsilon \frac{\partial E}{\partial t} \approx 0\right)$. In free space $z \leq 0$, then

$$
\nabla^{2} H_{z}(x, y, z \leq 0)=0
$$

The magnetic fields in free space can then be obtained by the upwardcontinuation of their surface values (Telford et. al.,1976),

$$
H_{z}\left(k_{x}, k_{y}, z \leq 0\right)=H_{z}\left(k_{x}, k_{y}, z=0\right) \exp \left(z \sqrt{k_{x}^{2}+k_{y}^{2}}\right) .
$$

As shown in figure (2.2), let

$$
H_{a}\left(k_{x}, k_{y}, z\right)=H_{z}\left(k_{x}, k_{y}, z=0\right) \exp \left(-|z| \sqrt{k_{x}^{2}+k_{y}^{2}}\right)
$$

then the total $H_{z}$ is the summation of a symmetric term $H_{a}$ and $H_{z}^{\prime}$ which exists only in the subsurface.

$$
H_{z}\left(k_{x}, k_{y}, z\right)=H_{a}\left(k_{x}, k_{y}, z\right)+H_{z}^{\prime}\left(k_{x}, k_{y}, z\right)
$$

where $H_{z}^{\prime}$ is causal with respect to the $z$-axis, or $H_{z}^{\prime}\left(k_{x}, k_{y}, z \leq 0\right)=0$.

Substituting the Fourier transform of equation (2.23) in equation (2.24),

$$
\begin{gathered}
\check{H}_{z}\left(k_{x}, k_{y}, k_{z}\right)=\frac{2 \sqrt{k_{x}^{2}+k_{y}^{2}}}{k_{x}^{2}+k_{y}^{2}+k_{z}^{2}} H_{z}\left(k_{x}, k_{y}, z=0\right)+\tilde{H}_{z}^{\prime}\left(k_{x}, k_{y}, k_{z}\right) . \\
f\left(k_{x}, k_{y}, k_{z}\right)=\frac{2 i}{\sqrt{k_{x}^{2}+k_{y}^{2}}} H_{z}\left(k_{x}, k_{y}, z=0\right)+f^{\prime}\left(k_{x}, k_{y}, k_{z}\right) .
\end{gathered}
$$

Solving for $\tilde{J}_{x}$ and $\tilde{J}_{y}$ in equation (2.13) and (2.14),

$$
\hat{j}_{x}\left(k_{x}, k_{y}, z\right)=U(z)\left[\frac{2 i k_{y}}{\sqrt{k_{x}^{2}+k_{y}^{2}}} h_{z}\left(k_{x}, k_{y}, z=0\right)+k_{y} f^{\prime}-k_{x} g\right]
$$



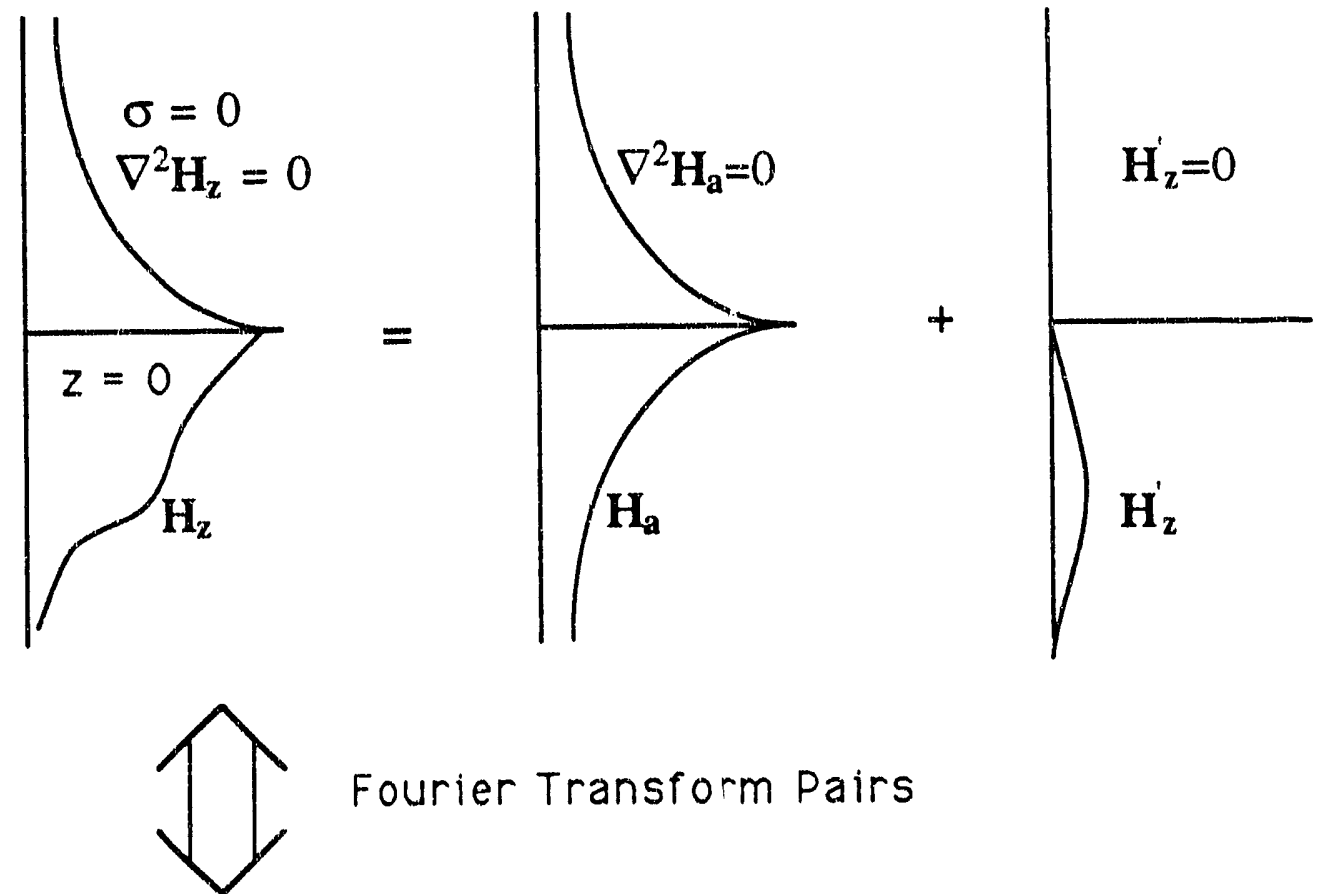

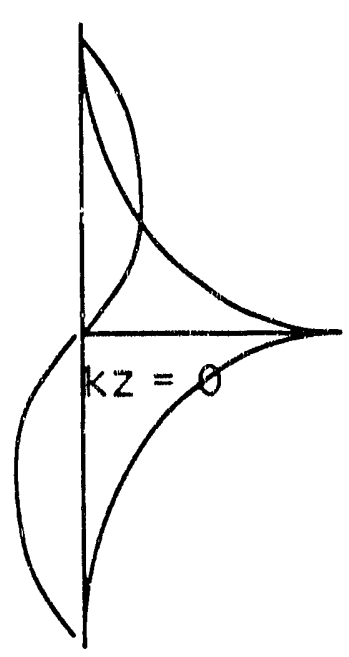

Real Even and Imaginary Odd

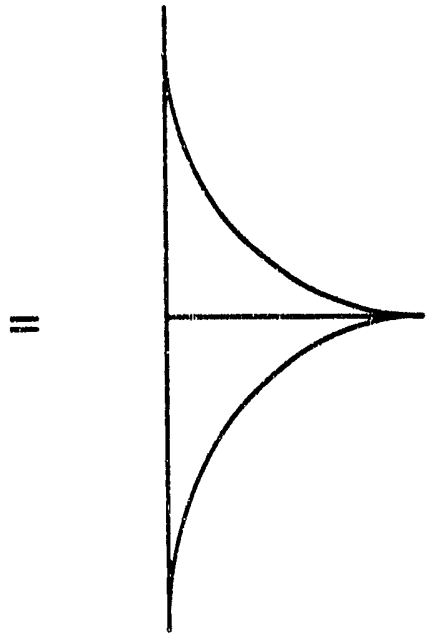

Real Even

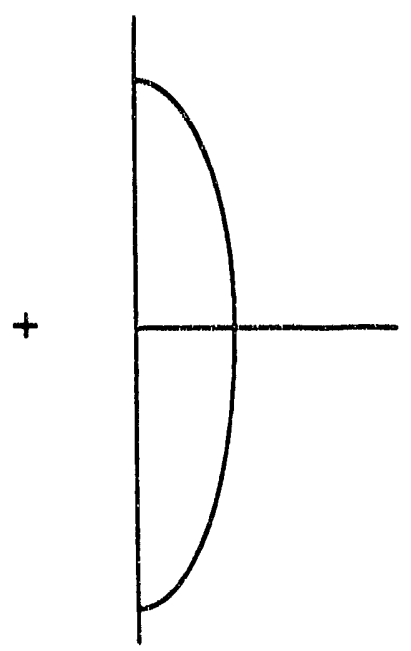

Real Even Imagingary odd

Figure 2-2 Fourier transform pairs for real, symmetric, and causal functions. 


$$
\tilde{J}_{y}\left(k_{x}, k_{y}, z\right)=U(z)\left[\frac{-2 i k_{x}}{\sqrt{k_{x}^{2}+k_{y}^{2}}} h_{z}\left(k_{x}, k_{y}, z=0\right)-k_{x} f^{\prime}-k_{y} g\right],
$$

where $U(z)$ is the unit step function.

Openheimer and Schafer (1975) shows that if a function is causal $\left(J_{x, y}(x, y, z<\right.$ $0))=0$ ) then it can be obtained either from even-harmonics, or from oddharmonics and the value at origin $\left(J_{x, y}(x, y, z=0)\right)$. Representing $\tilde{J}_{x, y}$ with even-harmonics with respect to $k_{z}$,

$$
\tilde{j}_{x, y}\left(k_{x}, k_{y},\left|k_{z}\right|\right)=\tilde{J}_{x, y}\left(k_{x}, k_{y}, k_{z}\right)+\tilde{J}_{x, y}\left(k_{x}, k_{y},-k_{z}\right),
$$

then

$$
\begin{gathered}
J_{x, y}(x, y, z)=U(z) j_{x, y}(x, y, z), \\
e_{x, y}(x, y, z \geq 0)=U(z) \rho(x, y, z) j_{x, y}(x, y, z) .
\end{gathered}
$$

Like $H_{z}, E_{x}$ and $E_{y}$ also satisfy Laplace's equation in free space,

$$
\begin{gathered}
\nabla^{2} E_{x, y}(x, y, z \leq 0)=0 \\
E_{x, y}\left(k_{x}, k_{y}, z \leq 0\right)=E_{x, y}\left(k_{x}, k_{y}, z=0\right) \exp \left(z \sqrt{k_{x}^{2}+k_{y}^{2}}\right) \\
\tilde{E}_{x, y}\left(k_{x}, k_{y}, k_{z}\right)=\frac{2 \sqrt{k_{x}^{2}+k_{y}^{2}}}{k_{x}^{2}+k_{y}^{2}+k_{z}^{2}} E_{x, y}\left(k_{x}, k_{y}, z=0\right)+\tilde{E}_{x, y}^{\prime}\left(k_{x}, k_{y}, k_{z}\right) .
\end{gathered}
$$

Since $J_{z}$ is causal with respect to the $z$-axis and $J_{z}(x, y, z=0)=0$, $E_{z}\left(k_{x}, k_{y}, k_{z}\right)$ can be obtained from either even or odd harmonics of Equation $(2.15)$.

\subsection{Dipoles in $\mathrm{t}-\mathrm{k}$ domain}

The analytic expression for the electric or magnetic dipole serves as an initial condition in the time-stepping method. Superpostion of electric and/or 
magnetic dipoles can simuluate any source configurations, so their characteristics are important for developing this time-stepping algorithm.

The unit step current response of the vertical electric current density $\left(J_{z}\right)$ or vertical magnetic field $\left(H_{z}\right)$ by a vertical electric dipole $\left(m_{e}\right)$ or magnetic dipole $\left(m_{m}\right)$ is given by (Ward and Hohmann, 1988):

$$
\begin{aligned}
\left(J_{z}, H_{z}\right)(\vec{r}, t) & =\frac{\left(m_{e}, m_{m}\right)}{4 \pi r^{3}}\left[\left(\frac{4}{\sqrt{\pi}} \theta^{3} r^{3}+\frac{6}{\sqrt{\pi}} \theta r\right) \exp \left(-\theta^{2} r^{2}\right)+3 \operatorname{erfc}(\theta r)\right) \frac{z^{2}}{r^{2}} \\
& \left.\left.-\left(\frac{4}{\sqrt{\pi}} \theta^{3} r^{3}+\frac{2}{\sqrt{\pi}} \theta r\right) \exp \left(-\theta^{2} r^{2}\right)+\operatorname{erf} c(\theta r)\right)\right]
\end{aligned}
$$

where

$$
\begin{gathered}
r=\sqrt{x^{2}+y^{2}+z^{2}} \\
\mu_{0}=\text { magnetic permeability, } \\
\sigma=\text { electric conductivity, } \\
m_{e}=\text { electric dipole moment } \\
m_{m}=\text { magnetic dipole moment } \\
\theta=\sqrt{\frac{\mu_{0} \sigma}{4 t}}
\end{gathered}
$$

The impulse current response can be obtanied by differentiating equation (2.35) with respect to time.

$$
\left(J_{z}, H_{z}\right)(\vec{r}, t)=\frac{\left(m_{e}, m_{m}\right) \theta^{3}}{\pi^{3 / 2} t} \exp \left(-\theta^{2} r^{2}\right)\left[1-\theta^{2}\left(x^{2}+y^{2}\right)\right] .
$$

Defining the Fourier transform pair by,

$$
\tilde{f}(\vec{k})=\int_{-\infty}^{\infty} f(\vec{r}) \exp (-i \vec{k} \cdot \vec{r}) d \vec{r}
$$




$$
f(\vec{r})=\frac{1}{8 \pi^{3}} \int_{-\infty}^{\infty} \tilde{f}(\vec{k}) \exp (i \vec{k} \cdot \vec{r}) d \vec{k}
$$

The wavenumber representation of the impulse response becomes

$$
\left(\tilde{J}_{z}, \tilde{H}_{z}\right)(\vec{k}, t)=\left(m_{e}, m_{m}\right) \frac{\left(k_{x}^{2}+k_{y}^{2}\right)}{\mu_{0} \sigma} \exp \left(-\frac{1}{\mu_{0} \sigma}\left(k_{x}^{2}+k_{y}^{2}+k_{z}^{2}\right)\right) .
$$

The unit step current responses are obtained by integrating equation (2.37) with respect to time.

$$
\left(\tilde{J}_{z}, \tilde{H}_{z}\right)(\vec{k}, t)=\left(m_{e}, m_{m}\right) \frac{\left(k_{x}^{2}+k_{y}^{2}\right)}{\left(k_{x}^{2}+k_{y}^{2}+k_{z}^{2}\right)} \exp \left(-\frac{t}{\mu_{0} \sigma}\left(k_{x}^{2}+k_{y}^{2}+k_{z}^{2}\right)\right) \text {. }
$$

The scalar potentials $f$ and $g$ for the unit step current are

$$
\begin{gathered}
f(\vec{k}, t)=i m_{m} \exp \left(-\frac{t}{\mu_{0} \sigma}\left(k_{x}^{2}+k_{y}^{2}+k_{z}^{2}\right)\right), \\
g(\vec{k}, t)=\frac{m_{e} k_{z}}{\left(k_{x}^{2}+k_{y}^{2}+k_{z}^{2}\right)} \exp \left(-\frac{t}{\mu_{0} \sigma}\left(k_{x}^{2}+k_{y}^{2}+k_{z}^{2}\right)\right) .
\end{gathered}
$$

For the impulse current response $g$ becomes

$$
g(\vec{k}, t)=\frac{m_{e} k_{z}}{\mu_{0} \sigma} \exp \left(-\frac{t}{\mu_{0} \sigma}\left(k_{x}^{2}+k_{y}^{2}+k_{z}^{2}\right)\right)
$$

Comparing the above equations, not only are the wavenumber expressions more fundamental than the spatial ones, but also they are invariant in time and space. i.e., a change in time can be treated as a change in wavenumber or vice versa. If $t^{\prime}=\alpha^{2} t$ then,

$$
\left(\tilde{J}_{z}, \tilde{H}_{z}\right)\left(\vec{k}, t^{\prime}\right)=\left(\tilde{J}_{z}, \tilde{H}_{z}\right)(\alpha \vec{k}, t)
$$

Since the superposition of electric and magnetic dipoles can simulate any kind of source configuration, such a source should have the same characteristics as the dipoles. Figure (2.4) to (2.8) show the unit step and impulse 


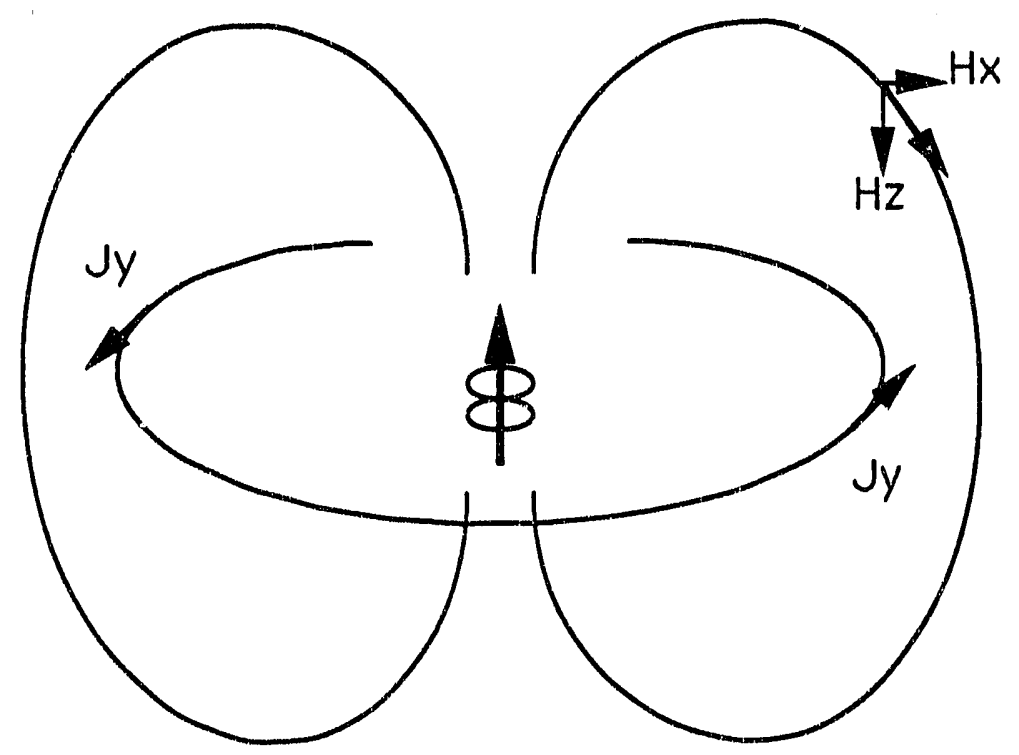

Vertical Magnetic Dipole (Mz)

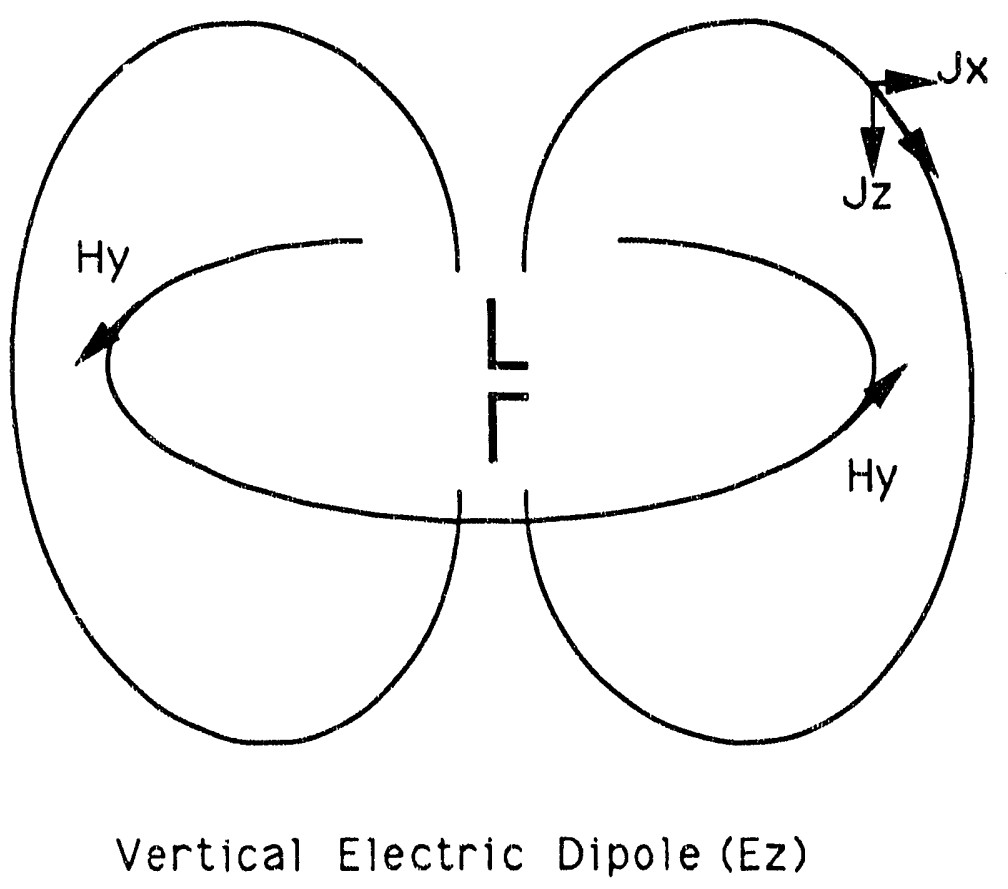

Figure 2-3 Schematic diagram of vertical magnetic and electric dipole fields. 

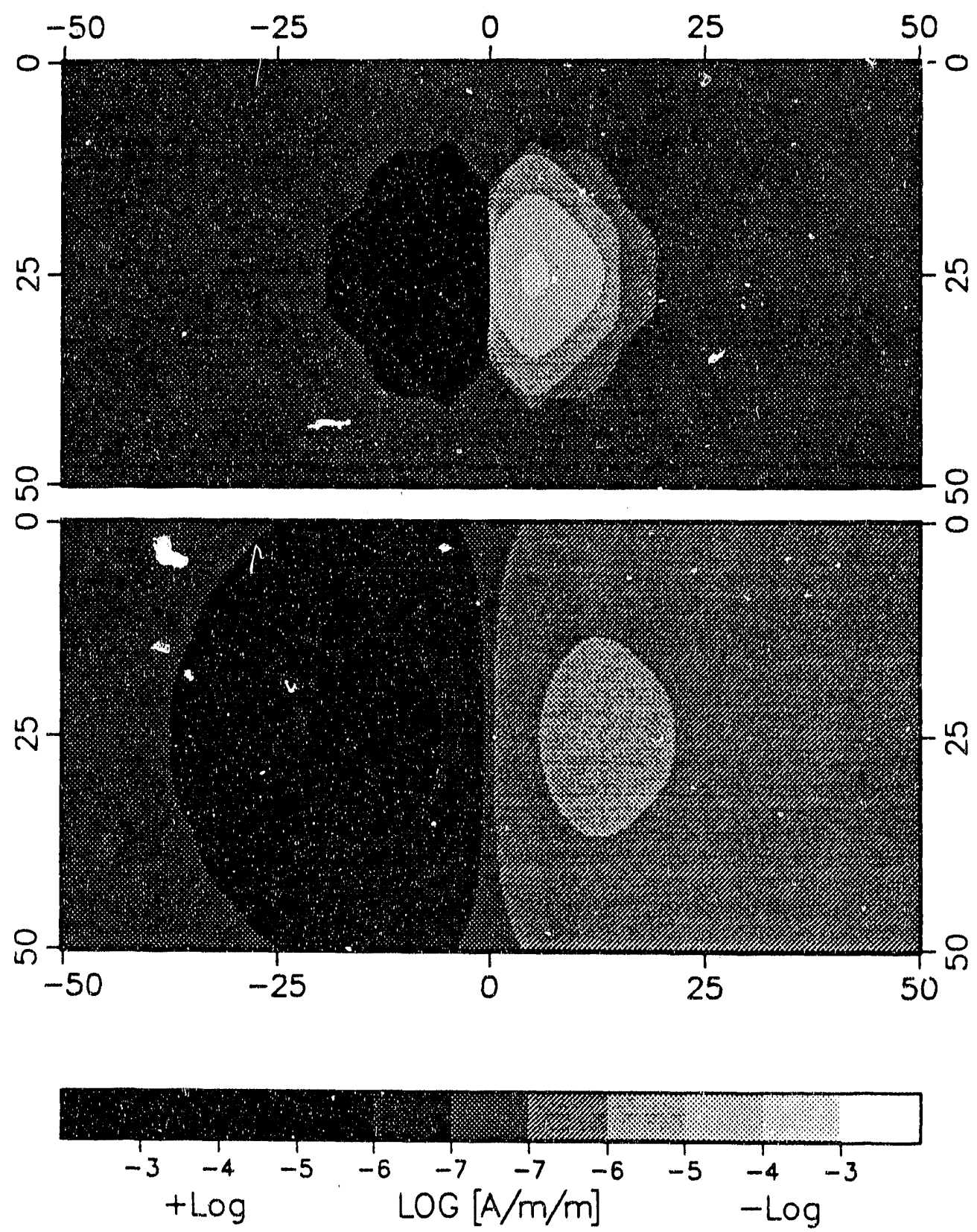

Figure 2-4 Horizontal current density $\left(J_{y}\right)$ for a step current magnetic dipole (the dipole is at the center of $10 \mathrm{Ohm} . \mathrm{m}$ full space, 1 and 10 usec snapshots). 

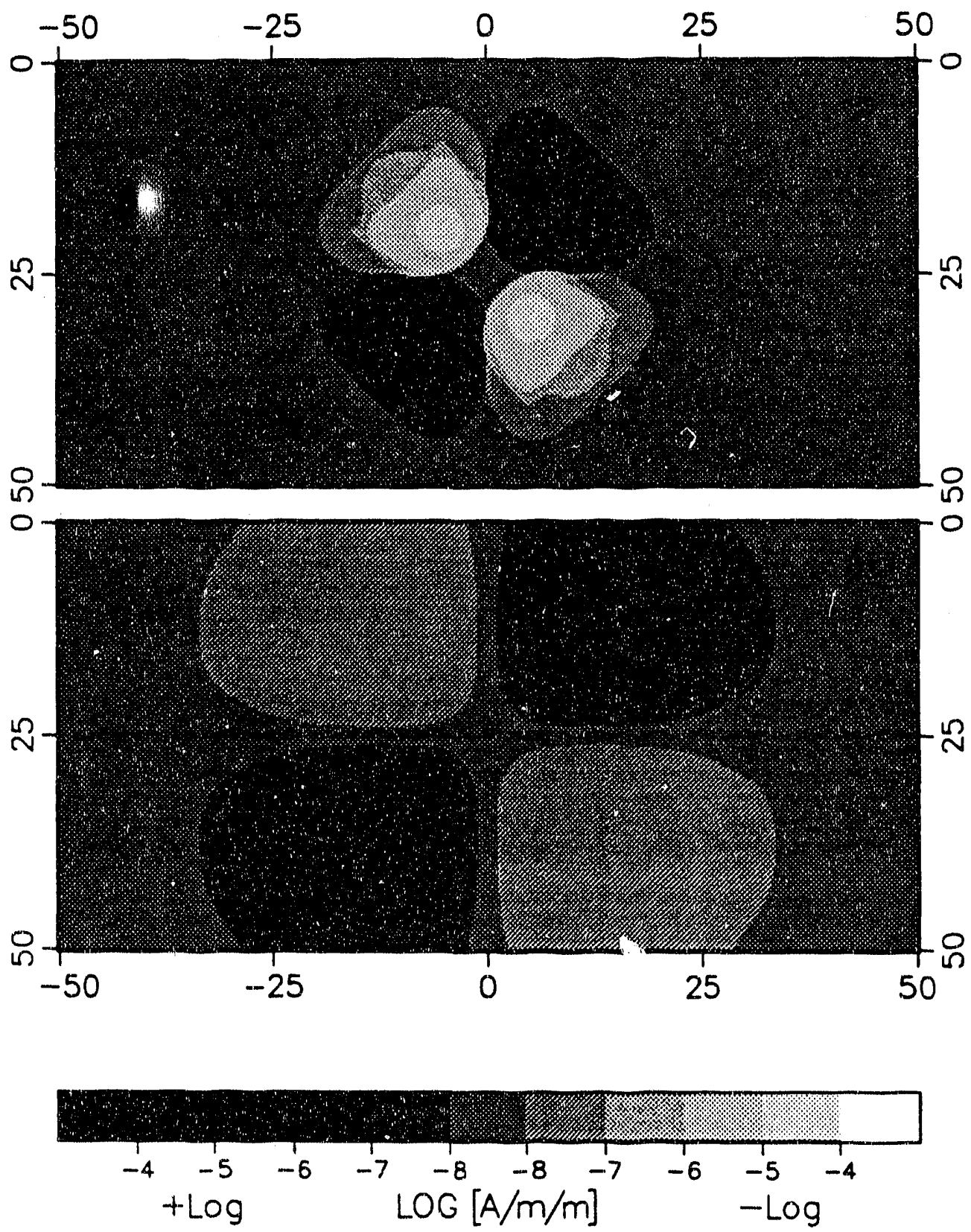

Figure 2-5 Horizontal current density $\left(\mathrm{J}_{\mathrm{X}}\right)$ for an impulse current electric dipole (the dipole is at the center of $10 \mathrm{Ohm} . \mathrm{m}$ full space, 1 and $10 \mathrm{usec}$ snapshots). 

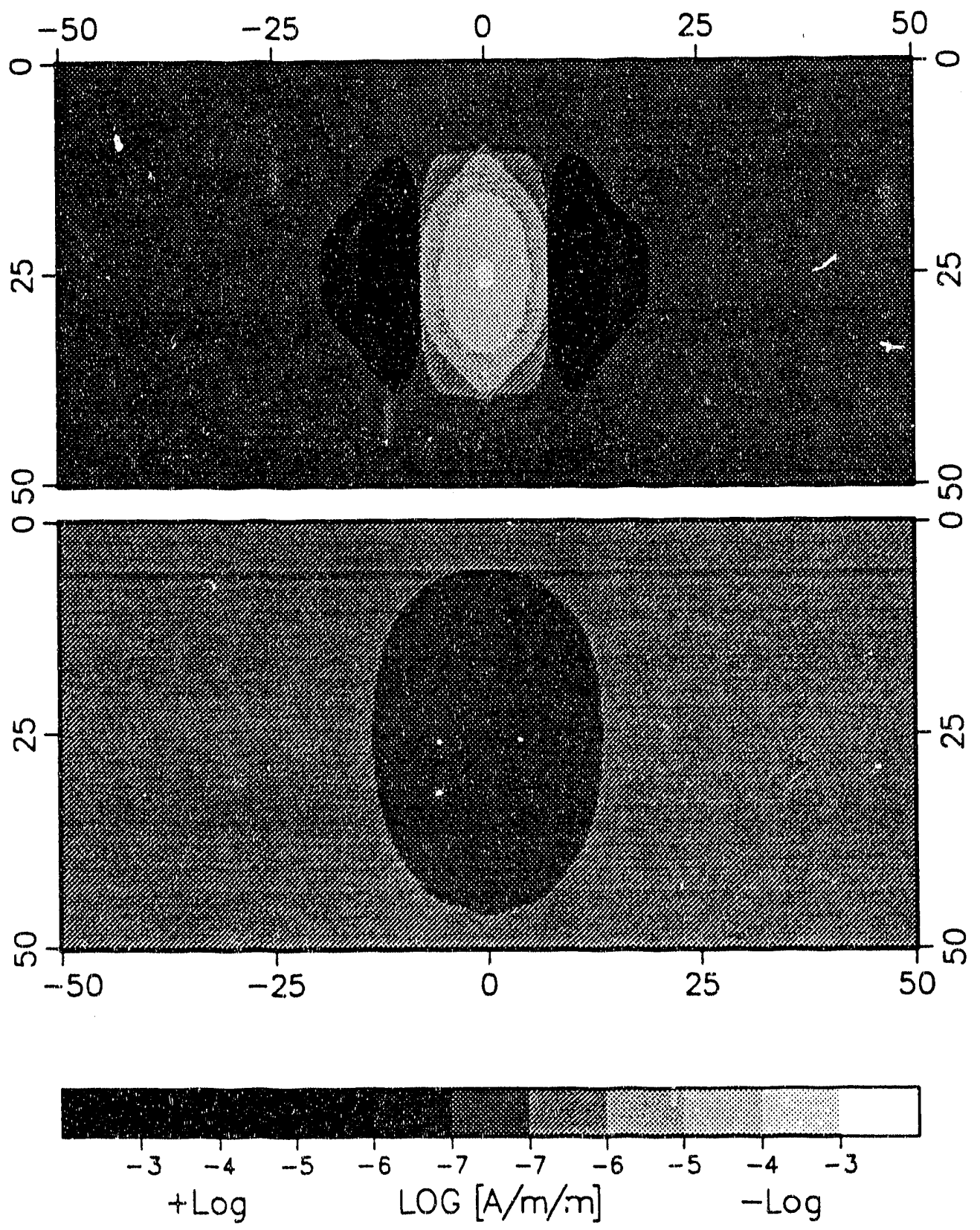

Figure 2-6 Vertical current density $\left(\mathrm{J}_{\mathrm{Z}}\right)$ for an impulse current electric dipole (the dipole is at the center of 10 Ohm.m full space, 1 and 10 usec snapshots). 

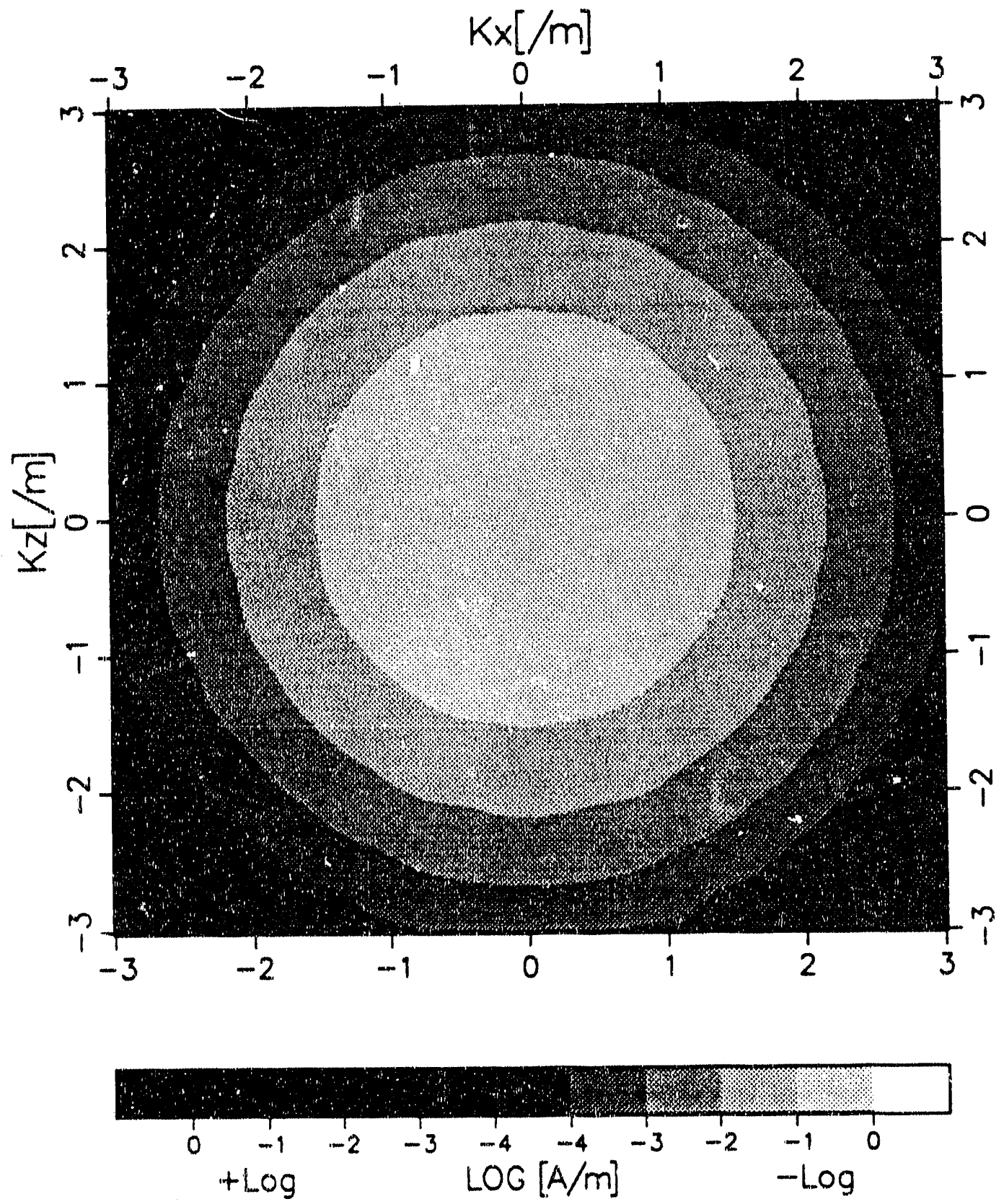

Figure 2.7 Scalar potential $f$ for a step current magnetic dipole. 

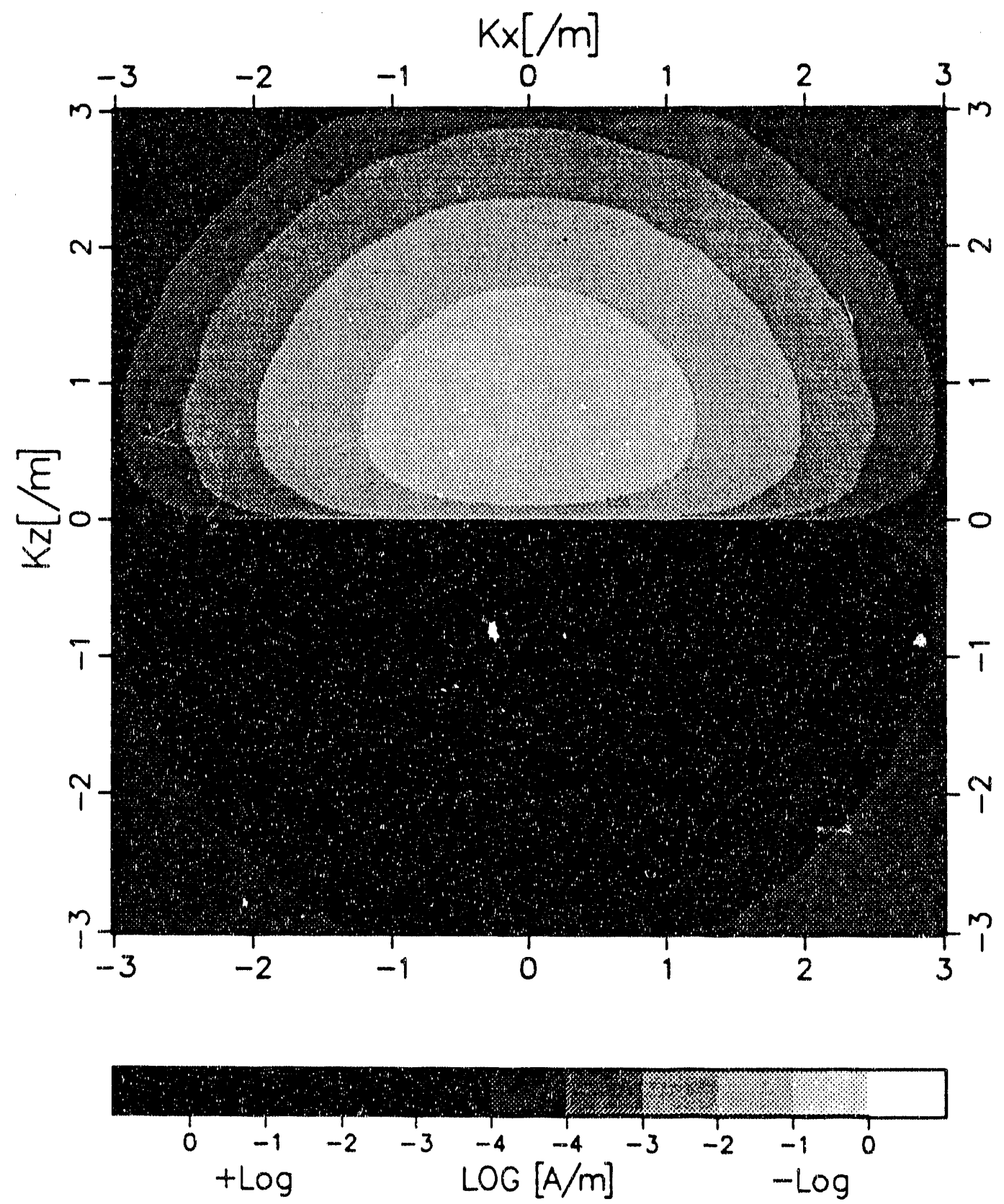

Figure 2-8 Scalar potential g for an impulse current electric dipole. 
current response in the $(x, z)$ and $\left(k_{x}, k_{z}\right)$ domain. For the potentials $\frac{t}{\mu_{0} \sigma}$ is set to one unit. The overall 3-dimensional patterns can be obtained by rotating these figures on the $z$ or $k_{z}$ axis.

\subsection{Singularity}

When $k_{x}=k_{y}=0$ the horizontal current densities $\tilde{J}_{x}$ and $\tilde{J}_{y}$ can not be obtained from equation (2.7) and (2.8) since they are divided by zero. But this singularity can be treated analytically if the sources are finite. From the definition of Fourier transform,

$$
\begin{aligned}
J_{z}\left(k_{x}=k_{y}=0, z, t\right) & =\int_{-\infty}^{\infty} \int_{-\infty}^{\infty} J_{z}(x, y, z, t) d x d y \\
& =\int_{S \rightarrow \infty} \vec{J}(x, y, z, t) \cdot d \vec{S}
\end{aligned}
$$

where $d \vec{S}=\hat{z} d x d y$. From Maxwell's equations,

$$
\begin{aligned}
J_{z}\left(k_{x}=k_{y}=0, z, t\right) & =\int_{S \rightarrow \infty}[\nabla \times \vec{H}(x, y, z, t)] \cdot d \vec{S} \\
& =\oint_{C \rightarrow \infty} \vec{H}(x, y, z, t) \cdot d \vec{l}
\end{aligned}
$$

where $S$ in an open surface bounded by the contour $C$, and $d \vec{l}$ is a line element on contour $\mathrm{C}$.

In the case of finite sources, an inhomogeneous medium can be enclosed in the finite volume $V_{o}$ sufficiently larger than the observation scale and surrounded by the homogeneous volume $V\left(\right.$ conductivity $\left.=\sigma_{0}\right)$ bounded by the contour $\mathrm{C}$, such that the volume $V_{o}$ is negligible compare to $V$. Therefore,

$$
J_{z}\left(k_{x}=k_{y}=0, z, t\right)=J_{z}\left(k_{x}=k_{y}=0, z, t, \sigma_{o}\right)
$$


In the case of $H_{z}$,

$$
\begin{aligned}
H_{z}\left(k_{x}=k_{y}=0, z, t\right) & =1 / \mu_{0} \int_{S \rightarrow \infty} \nabla \times\left[\int_{0}^{t} \vec{E}(x, y, z, t) d t\right] \cdot d \vec{S} \\
& =1 / \mu_{0} \int_{0}^{t} \oint_{C \rightarrow \infty} \vec{E}(x, y, z, t) \cdot d \vec{l} \\
& =H_{z}\left(k_{x}=k_{y}=0, z, t, \sigma_{o}\right)
\end{aligned}
$$

For the impulse current source, from equation (2.37)

$$
H_{z}\left(k_{x}=k_{y}=0\right)=J_{z}\left(k_{x}=k_{y}=0\right) \equiv 0
$$




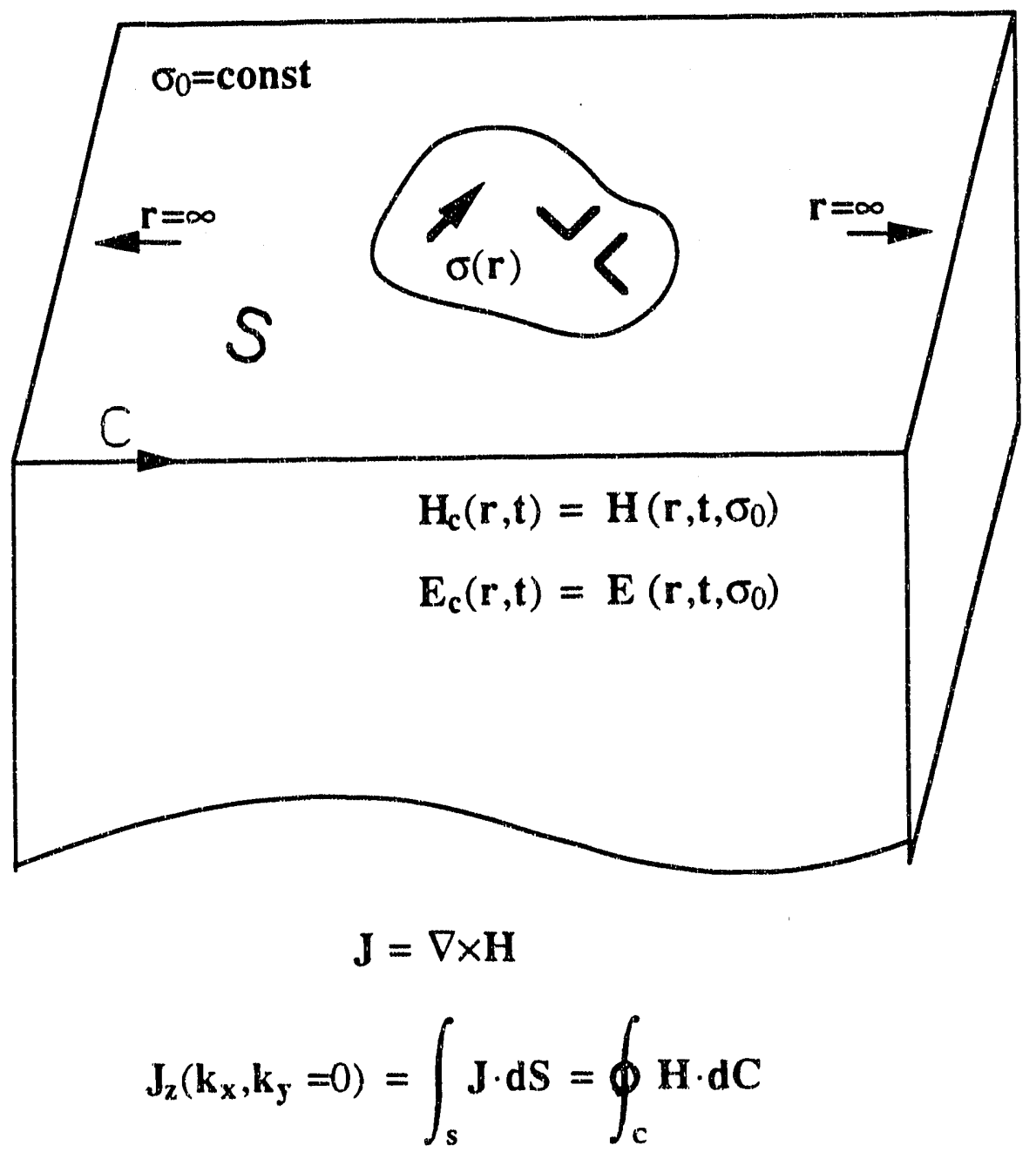

Figure 2-9 Transformation of a surface integral to a line integral using Stoke's theorem. 


\section{Numerical Considerations}

In this chapter the numerical aspects of the $\mathrm{t}-\mathrm{k}$ algorithm will be considered. New approaches toward the time-stepping method and a numerical boundary condition developed using the nature of the diffusion process in the wavenumber domain will be discussed.

\subsection{Modified forward time-stepping}

In scalar differential equations, there are numerous ways to approximate the time derivatives for initial value problems (Richtmyer and Morton, 1967). For coupled partial differential equations with a huge number of nodes, a method that requires the inversion of matrices (implicit method) has to be excluded. For example, the full matrix arising from a $50 \times 50 \times 50 \mathrm{mesh}$ requires $50^{9}$ calculations to invert using a direct approach (e.g. Gaussian elimination method), and somewhat less (still order of $50^{6}$ ) assuming that an iterative method (e.g. relaxation) converges.

For explicit methods that do not require matrix inversion, the forward approximation of the time derivative (Euler's method; Mitchell and Griffiths (1980)) can be applied to all numerical algorithrns. To improve the efficiency of these algorithms, the leap-frog method for 3-D Maxwell's equations in a non conducting space domain (Yee, 1966) and the Dufort-Frankel method for the 2-D diffusion of electromagnetic fields in the conductive space domain (Oristaglio and Hohmann, 1984) have been successfully implemented so far. The coupled electric and magnetic vector fields in the 3-D Maxwell's equations disrupt the linear relationship leading to an explicit formula that 
can be used with the Dufort-Frankel method. So this approach also results in matrix inversion.

Euler's method of time differencing is always explicit with a well defined stability criteria. However the stability criteria demands impractically short time steps especially if late time solutions are required. Here a characteristic of the diffusion fields at late time will be used to relax this restriction. Euler's method is defined by faking the first term from the Taylor's series expansion,

$$
\begin{gathered}
f(t+\delta t)=f(t)+\delta t \frac{\partial f(t)}{\partial t}+\frac{\delta t^{2}}{2 !} \frac{\partial^{2} f(t)}{\partial t^{2}}+\cdots, \\
f(t+\delta t) \approx f(t)+\delta t \frac{\partial f(t)}{\partial t} .
\end{gathered}
$$

In the $t-k$ algorithm the first time derivative can be written as,

$$
\begin{aligned}
& \frac{\partial f(\vec{k}, t)}{\partial t}=-L_{f}(f(\vec{k}, t), g(\vec{k}, t)), \\
& \frac{\partial g(\vec{k}, t)}{\partial t}=-L_{g}(f(\vec{k}, t), g(\vec{k}, t)),
\end{aligned}
$$

where the linear operator $L_{f}$ and $L_{g}$ represent the numerical steps required to reach equation (2.16) and (2.17).

In a homogeneous medium these linear operators become

$$
\begin{aligned}
& \frac{\partial f(\vec{k}, t)}{\partial t}=-\frac{|\vec{k}|^{2}}{\mu \sigma} f(\vec{k}, t) . \\
& \frac{\partial g(\vec{k}, t)}{\partial t}=-\frac{|\vec{k}|^{2}}{\mu \sigma} g(\vec{k}, t) .
\end{aligned}
$$

For this expression, the Von Newmann stability criterion (Mitchell and Griffiths, 1980) which gurantees convergence is that

$$
\delta t \leq \min \left[\frac{\mu \sigma}{\left(k_{x}^{2}+k_{y}^{2}+k_{z}^{2}\right)}\right] .
$$


For equal grid-spacing $\delta x, \delta y$, and $\delta z$, this can also be written as

$$
\delta t \leq \frac{\mu \sigma}{\pi^{2}\left(\delta x^{-2}+\delta y^{-2}+\delta z^{-2}\right)}
$$

The maximum time step is inversely proportional to the square of gridspacing.

Oristaglio and Hohmann (1984) showed that, in gophysical problems, the stability criteria of Euler's method must be used at early times in order to track the rapid change of the fields. But this time step is not practical at late times when the fields change smoothly. As shown in chapter (2.3), the scalar potentials for the electric and magnetic dipoles are

$$
\begin{aligned}
& f(\vec{k}, t+\delta t)=\exp \left(-\delta t \frac{|\vec{k}|^{2}}{\mu \sigma}\right) f(\vec{k}, t), \\
& g(\vec{k}, t+\delta t)=\exp \left(-\delta t \frac{|\vec{k}|^{2}}{\mu \sigma}\right) g(\vec{k}, t) .
\end{aligned}
$$

If a function decays exponentially, then the logarithm of the function is linear and its derivative becomes

$$
\frac{\partial \log f(t)}{\partial t}=\frac{1}{f} \frac{\partial f(t)}{\partial t}
$$

This concept can be derived more regiously. Noting that the series expansion of

$$
e^{x}=1+x+\frac{x^{2}}{2 !}+\cdots
$$

Then equation (3.1) becomes (Mitchell and Griffiths, 1980)

$$
f(t+\delta t)=\left[\exp \left(\delta t \frac{\partial}{\partial t}\right)\right] f(t)
$$


Defining operator $\frac{\partial}{\partial t}$ in the $t-k$ algorithm,

$$
\begin{array}{ll}
\frac{\partial_{f}}{\partial t}=-\frac{L_{f}(f(\vec{k}, t), g(\vec{k}, t))}{f(\vec{k}, t)} & (f(\vec{k}, t) \neq 0), \\
\frac{\partial_{g}}{\partial t}=-\frac{L_{g}(f(\vec{k}, t), g(\vec{k}, t))}{g(\vec{k}, t)} \quad(g(\vec{k}, t) \neq 0),
\end{array}
$$

Approximating these operators as actual finite differences,

$$
\begin{aligned}
& f(\vec{k}, t+\delta t)=\exp \left(-\delta t \frac{\partial_{f}}{\partial t}\right) f(\vec{k}, t), \\
& g(\vec{k}, t+\delta t)=\exp \left(-\delta t \frac{\partial_{g}}{\partial t}\right) g(\vec{k}, t),
\end{aligned}
$$

In the homogeneous medjum,

$$
\begin{aligned}
& f(\vec{k}, t+\delta t)=\exp \left(-\delta t \frac{|\vec{k}|^{2}}{\mu \sigma}\right) f(\vec{k}, t), \\
& g(\vec{k}, t+\delta t)=\exp \left(-\delta t \frac{|\vec{k}|^{2}}{\mu \sigma}\right) g(\vec{k}, t) .
\end{aligned}
$$

As shown in the above equations, these expressions are the exact representation of any arbitrary distribution of dipoles in a homogeneous medium with arbitrary time steps. So this new approach in time-stepping satisfies the Von Newmann stability criteron with unconditional stability.

In an inhomogeneous medium, there is no unique way to approximate the time derivatives. Only short enough time intervals guarantee more accurate solutions. But, as Harringtion (1961) mentioned in his "Equivalance Principle" (Figure 3.1), the response from an inhomogeneity is equivalent to the electric or magnetic boundary current distribution at the boundary. The superposition of electric or magnetic dipoles is equivalent to the electric or 


\section{ORIGINAL PROBLEM}
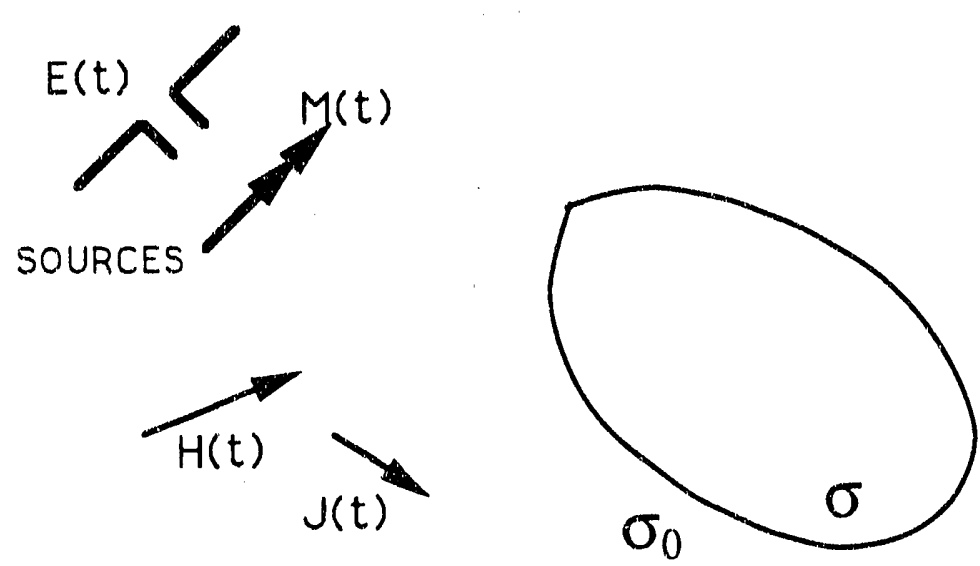

\section{EQUIVALENT PROBLEM}
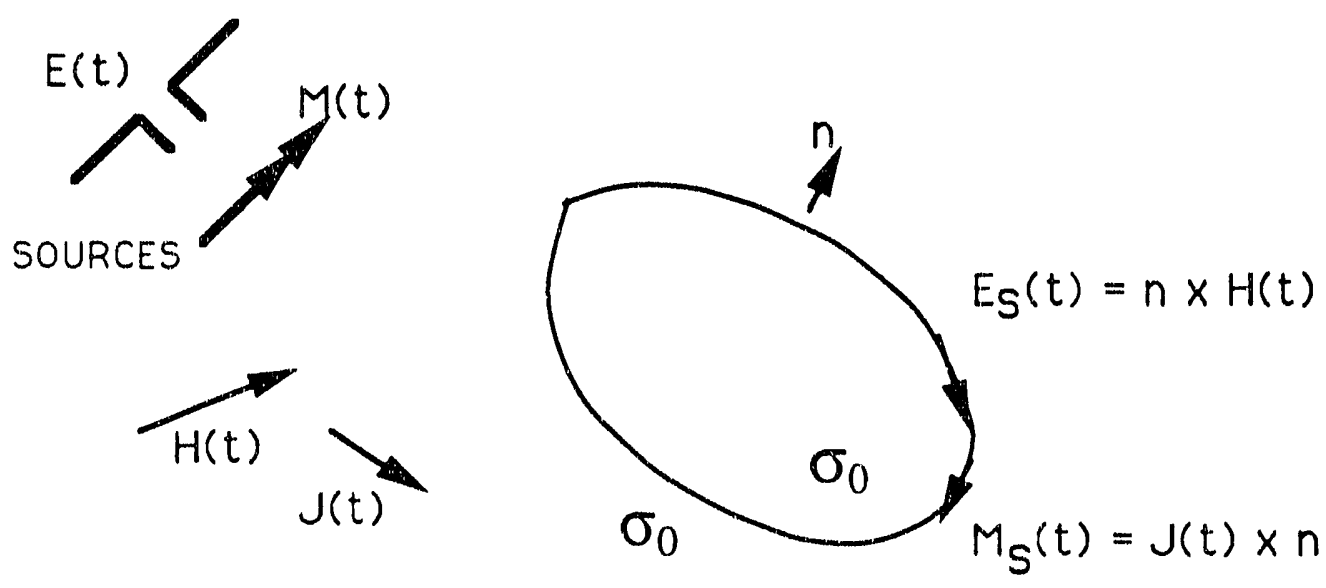

Figure 3-1 Equivalence principle (after Harrington, 1961). 
magnetic current distibution. i.e., in the time domain, it is equivalent to the superposition of time-dependent a electric or a magnetic dipole distributions. In the homogeneous medium an arbitrary distribution of magnetic dipole can be represented as

$$
f(\vec{k}, t+\delta t)=M(\vec{k}) \exp \left(-\delta t \frac{|\vec{k}|^{2}}{\mu \sigma}\right) f(\vec{k}, t),
$$

where $M(\vec{k})$ is the constant representing the location and magnitude of magnetic dipoles. In the inhomogeneous medium, $M(\vec{k})$ is no longer independent of time.

$$
f(\vec{k}, t)=M(\vec{k}, t) \exp \left(-t \frac{|\vec{k}|^{2}}{\mu \sigma}\right) .
$$

The time-derivative becomes,

$$
\frac{\partial f}{\partial t}=\frac{\partial M(\vec{k}, t)}{\partial t} \exp \left(-t \frac{|\vec{k}|^{2}}{\mu \sigma}\right)+M(\vec{k}, t) \frac{\partial}{\partial t} \exp \left(-t \frac{|\vec{k}|^{2}}{\mu \sigma}\right) .
$$

When the coefficient $M(\vec{k}, t)$ can be assumed to be smoothly time-varying in the diffusion process. then

$$
\frac{\partial f}{\partial t} \approx M(\vec{k}, t) \frac{\partial}{\partial t} \exp \left(-t \frac{|\vec{k}|^{2}}{\mu \sigma}\right)
$$

This modified time-stepping method is exact for the above approximation. The numerical calculation of the first term in equation (3.13) has to be done over short enough time intervals but this approach is better in the wavenumber domain diffusion process than Euler's method where both terms have to be produced by the finite time differences. 


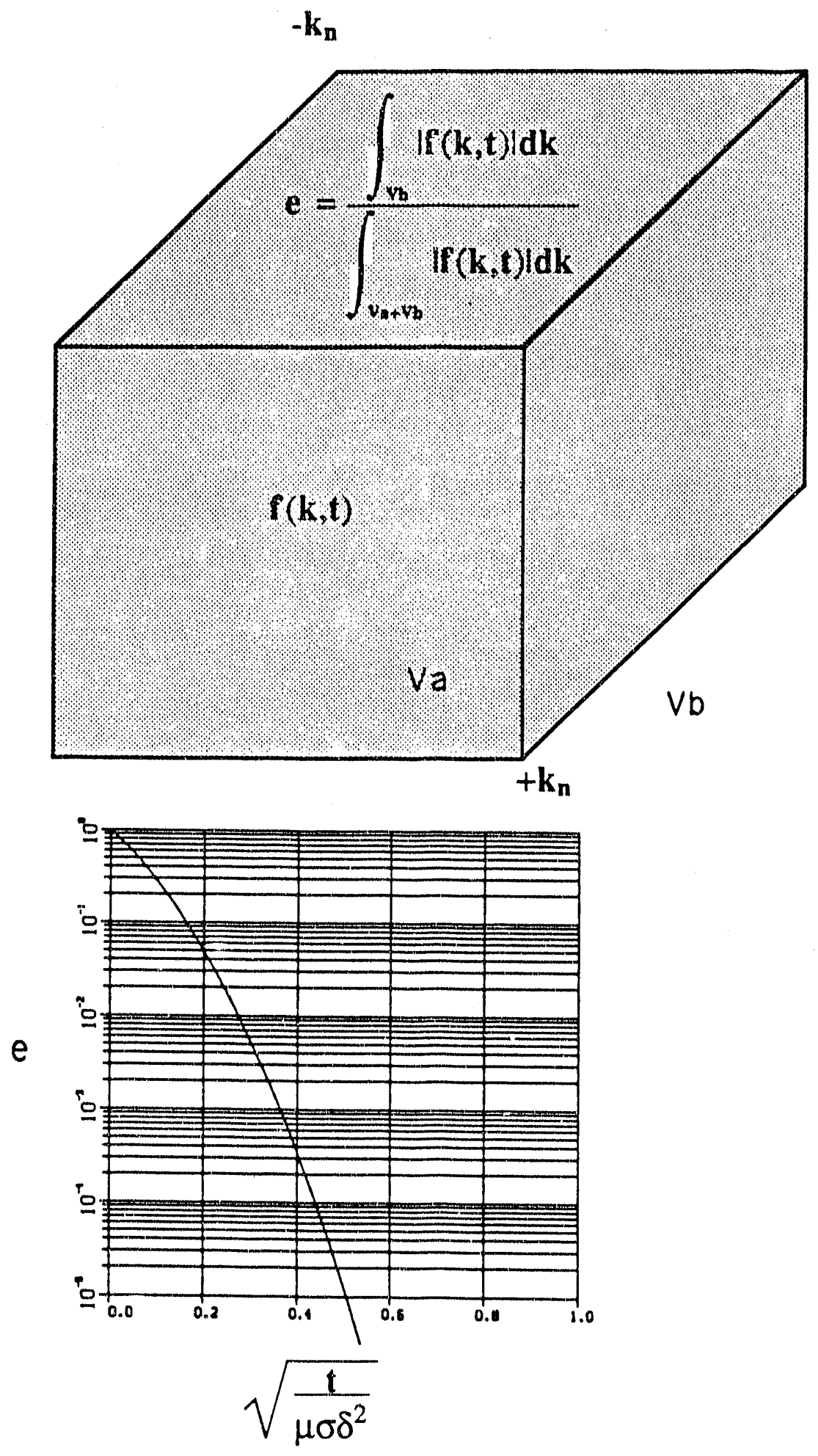

Figure 3-2 Relative error in the spectral amplitude of the potential $f$ and $g$ as a function of finite grid-spacing. 


\subsection{Numerical bowridary and grid-spacing}

In numerical formulations, there always is an artificial boundary created by the finite discretization of the medium (numerical boundary). These artificial boundaries influence the region of computation in one way or another and strongly affect the actual solution during the computation.

For wave propagation, there are many elegant ways to treat these boundaries, such as the absorbing boundary condition (Engquist and Majda, 1977), or one can simply stop the computation when the fields reach the boundary (pseudo-spectral method) since the response of interest is usually over by the time the fields reach the boundaries. There has been an unsuccessful attempt to adopt the absorbing boundary condition to the diffusion process (Adhidya et al, 1985).

Waves which propagate with finite velocity can be focused to a single location with proper design of the inhomogeneity (optical lenses, etc.). Likewise, a wave field can be redirected so as not to interfere in the region of interest by a proper design of numerical boundaries. Since we have neglected the dielectric constant $(\epsilon)$, the electromagetic wave diffuses instantaneously.

One way to avoid this problem is always to place the numerical boundary far away from the region where the major diffusion process takes place (moving numerical boundary). The algorithm that has been described requires the forward and inverse Fourier transforms at each time step. The discrete Fourier transform can be evaluated from irregularly spaced points so the grid-spacing can be gradually increased in order to place the numerical boundaries far away from the region of interest. With this scheme the 
number of calculation required $\left(2 N^{2} ; N=\right.$ number of nodes $)$ becomes too costly for a large data set. On the other hand, the number of calculations for the fast Fourier transform is $\left(N \log _{2} N\right)$. But the grid-spacing has to be regular and the numerical boundary appears in the region of computation as an aliasing effect when the diffusion process reaches the numerical boundary.

Let a 3-dimensional homogeneous medium be discretized by the gridspacing,

$$
\delta \vec{r}=(\delta x, \delta y, \delta z),
$$

then the Nyquist wavenumbers become,

$$
\overrightarrow{k_{n}}=\left(\frac{\pi}{\delta x}, \frac{\pi}{\delta y}, \frac{\pi}{\delta z}\right)
$$

Defining the truncation error $(e)$ as a ratio of truncated amplitude to the total amplitude at time $t$,

$$
e(t)=\frac{\int_{k_{n}}^{\infty} M(\vec{k}, t) \exp \left(-|\vec{k}|^{2} \frac{t}{\mu \sigma}\right) d \vec{k}}{\int_{0}^{\infty} M(\vec{k}, t) \exp \left(-|\vec{k}|^{2} \frac{t}{\mu \sigma}\right) d \vec{k}},
$$

For the potentials $f$ and $g$ in a homogeneous medium with a regular gridspacing $\delta$

$$
e(\delta, t)=\left[\operatorname{erfc}\left(\sqrt{\frac{t}{\mu \sigma}} \frac{\pi}{\delta}\right)\right]^{3}
$$

Figure (3.2) shows $e(t)$ as a function of $\delta$.

The expansion of grid-spacing can easily be implemented using the Fast Fourier transforms. Let

$$
F_{l, m, n}(\delta x, \delta y, \delta z)=F F T^{-1}\left[f\left(k_{x}, k_{y}, k_{z}\right)\right]
$$

then

$$
F_{l, m, n}(\alpha \delta x, \beta \delta y, \gamma \delta z)=F F T^{-1}\left[f\left(k_{x} / \alpha, k_{y} / \beta, k_{z} / \gamma\right)\right]
$$


At time $t^{\prime}=\alpha^{2} t, \delta^{\prime}=\alpha \delta$,

$$
e\left(\delta^{\prime}, t^{\prime}\right)=e(\delta, t)
$$

So without losing accuray in wavenumber amplitude, at late time $\alpha^{2} t$, one can increase the grid-spacing by a factor of $\alpha$. With the same discretization, the region of calculation and the numerical boundary can be increased by a factor of $\alpha$, and the stable time-stepping interval in Euler's method can be increased by a factor of $\alpha^{2}$ (equation 3.6). Figure (3.3) shows the snapshots of the horizontal current density for a vertical magnetic dipole where the gridspacing was increased by a factor of 3 when the time increased by a factor of 10 . In each case the total number of nodes is remained same $(64 \times 64 \times 32)$.

\subsection{Duration of computation}

For a time-stepping algorithm the field has to start from a known time domain solutions, usually an analytic solution for the full or half space. But, since we have neglected the dielectric constant $(\epsilon=0)$, the diffusion field exists everywhere after $t=0$.

The full space Green's function $G(r, t)$ for the pure diffusion equation satisfies (Ward and Hohmann, 1988)

$$
\nabla^{2} G+\mu \sigma \frac{\delta G}{\delta t}=\delta(x) \delta(y) \delta(z),
$$

and is given by

$$
G(r, t)=\frac{(\mu \sigma)^{1 / 2}}{8(\pi t)^{3 / 2}} \exp \left(-\mu \sigma r^{2} / 4 t\right) u(t)
$$




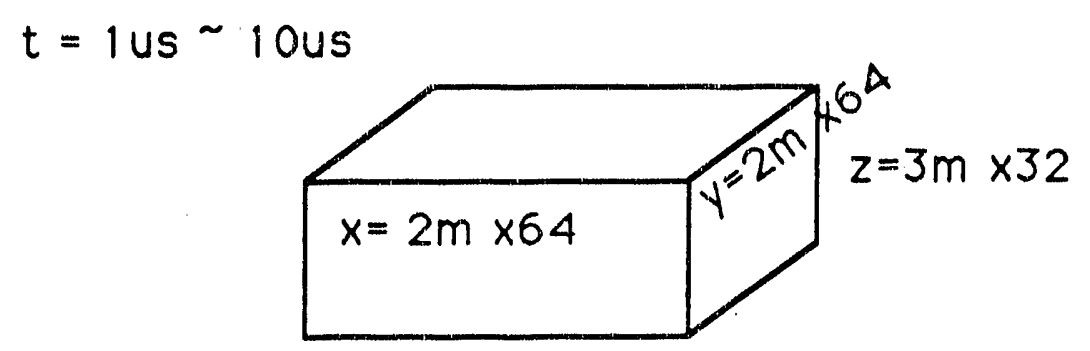

$t=10$ us $\sim 100 u s$

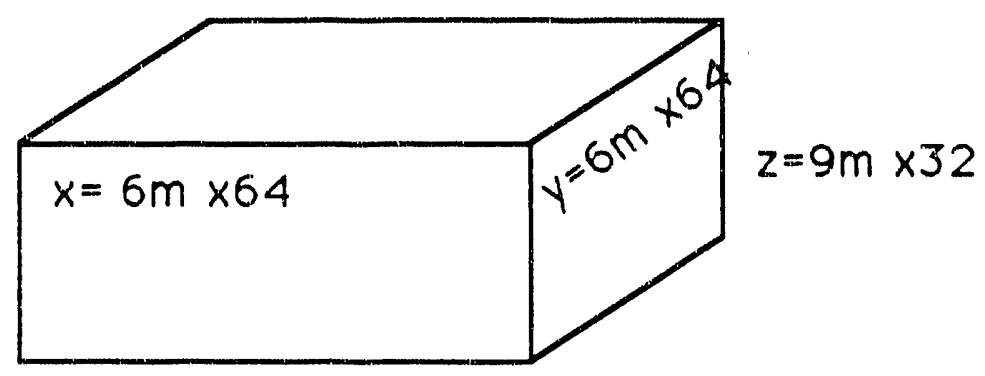

$t=100 u s \sim 1 \mathrm{~ms}$

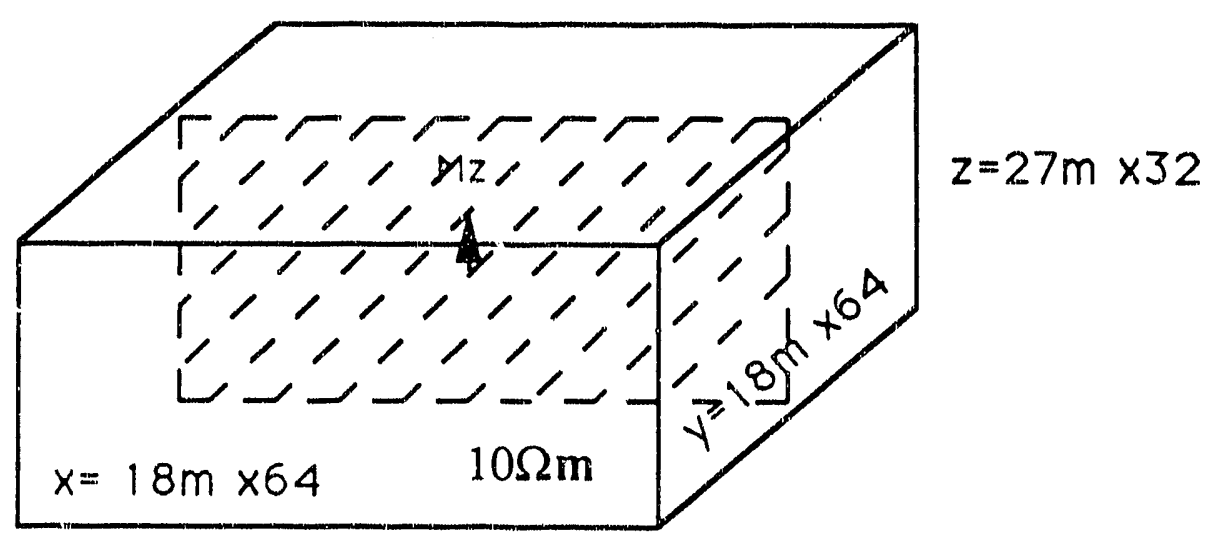

Figure 3-3 (a) Grid-spacing and numerical boundary for each decade. A vertical magnetic dipole is at the center of grid. Each volume contains $64 \times 64 \times 32$ nodes. 

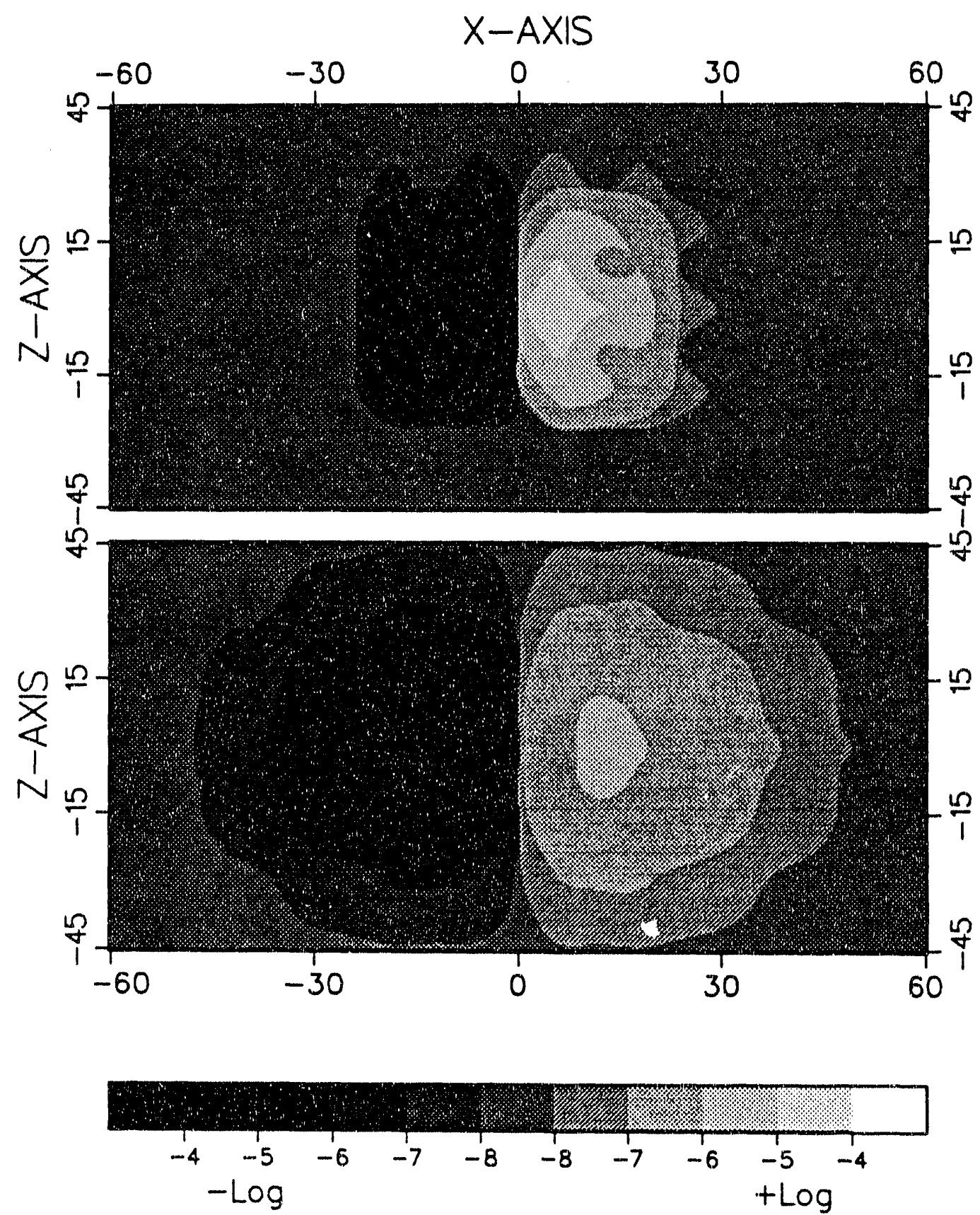

Figure 3-3 (b) Snapshots of the tangential current density $\left(\mathrm{J}_{\mathrm{y}}\right)$ at the starting and ending time ( 1 and 10 usec) of computation at the first grid configuration. The minor asymmetry in the figure is an artifact of the plotting software. 


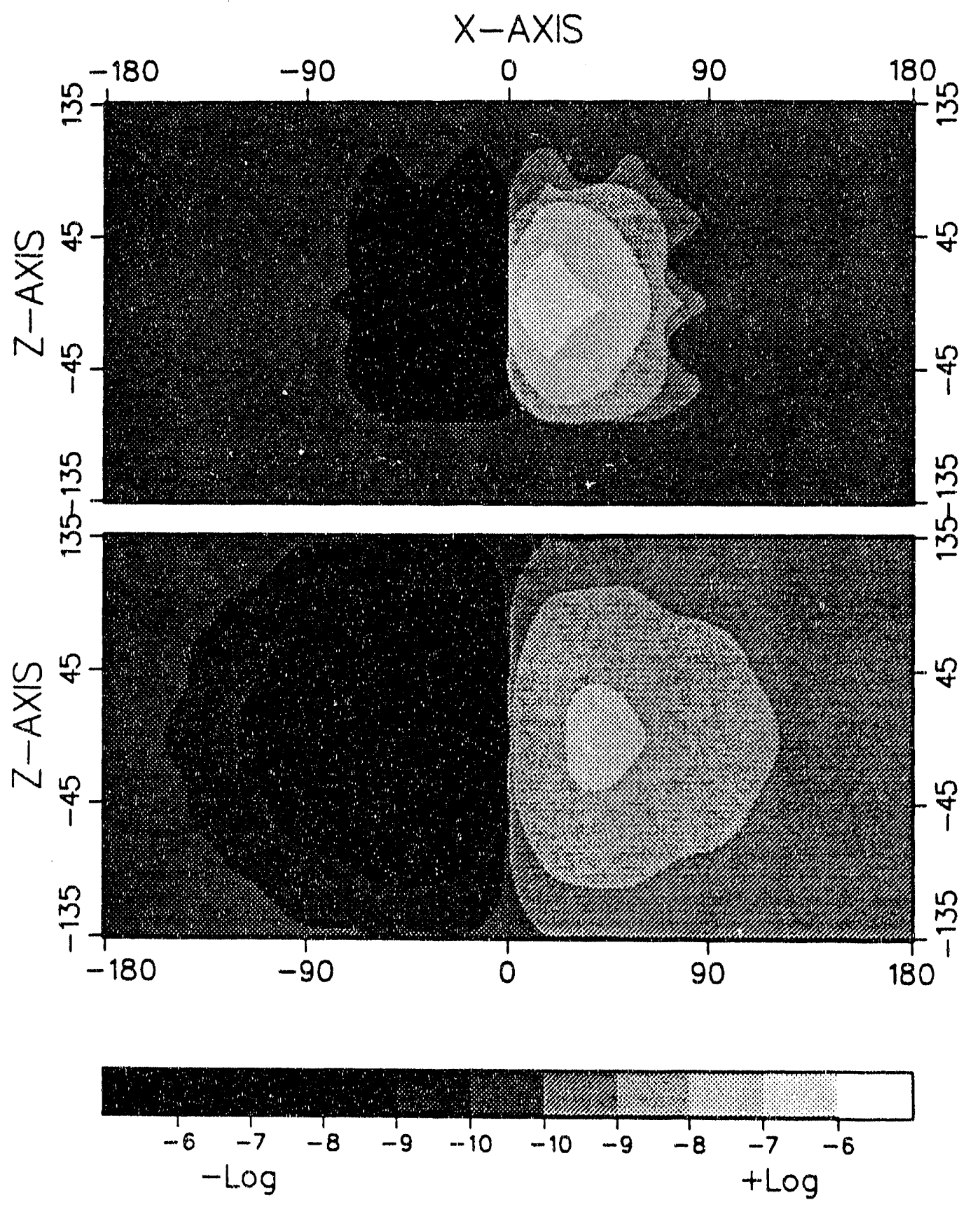

Figure 3-3 (c) Snapshots of $\mathrm{J}_{\mathrm{y}}$ at 10 and 100 usec at 2 nd decade. 


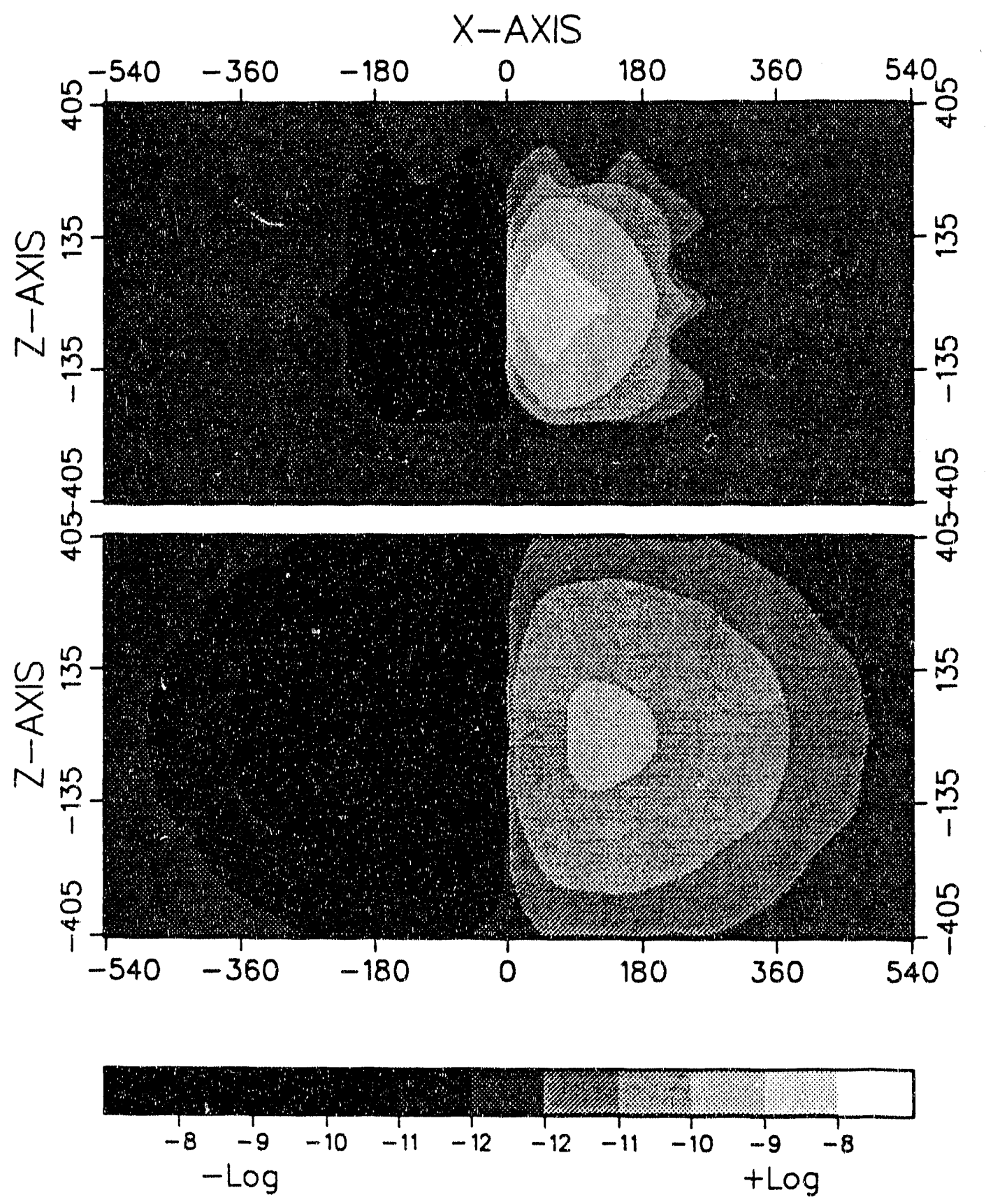

Figure 3-3 (d) Snapshots of $\mathrm{J}_{\mathrm{y}}$ at 100 and 1000 usec at 3rd decade. 
The Green's function for the wave equation satisfies (Ward and Hohmann, 1988)

$$
\nabla^{2} G+\mu \sigma \frac{\delta G}{\delta t}+\mu \epsilon \frac{\delta^{2} G}{\delta t^{2}}=\delta(x) \delta(y) \delta(z)
$$

and is given by

$$
G(r, t)=\frac{1}{4 \pi r}\left[\exp \left(-a t_{0}\right) \delta\left(t-t_{0}\right)+\frac{a t_{0} \exp (-a t)}{\left(t^{2}-t_{0}^{2}\right)^{1 / 2}} I_{1}\left(a\left(t^{2}-t_{0}^{2}\right)^{1 / 2}\right) u\left(t-t_{0}\right)\right]
$$

where $a=\sigma / 2 \epsilon$ and $t_{0}=r / v$. Figure (3.4) shows these Green's functions in $100 \Omega m$ whole space with $r^{r}=100 m$.

As shown in the previous chapter, the magnetic field always exists in the wavenumber domain and does not need to be confined in a homogeneous medium, but the electric current density has to be confined in the homogeneous medium before it diffuses to the inhomogeneous region since the electric field is calculated in the space domain. At very early times, away from the transmitter, these diffusion fields cannot be evaluated by this timestepping algorithm. But they do not need to be evaluated because not only are they very small but they are normally not measured in circumstances where the diffusion approximation breaks down. Even with the maximun medium dielectric constant ( $\epsilon=80 \epsilon_{0}$ for pure water), the submicrosecond time interval does not need to be evaluated in most geophysical (Figure 3.4) situations.

The calculations could be started at very early times, but this would require a large number of time steps to get to the desired time in the transient, and numerical errors, such as round-off and truncation errors, can quickly. overwhelm the true solution. Defining the diffusion radius in equation (3.21) 
as that for a "Smoke Ring" (Nabighian, 1979),

$$
r=\sqrt{4 t / \mu \sigma}[m]
$$

then it would seem that a reasonable initial time for this algorithm is the time when the distance from the source to the nearest inhomogeneous boundary is greater than $2 r$. When the region of interest is less than $r / 2$, it is not necessary to continue the time-stepping since the slope of decaying fields is independent of the conductivity distribution (Kaufman and Keller, 1983). Figure (3.5) shows the schematic of this concept. 


$$
\nabla^{2} \mathbf{G}+\mu \sigma \frac{\partial \mathbf{G}}{\partial \mathbf{t}}+\left(\mu \varepsilon \frac{\partial^{2} \mathbf{G}}{\partial \mathbf{t}^{2}}\right)=0
$$

$$
\delta(\mathbf{r}) \stackrel{100 \mathrm{~m}}{\rho=100 \Omega \mathrm{m} \quad \varepsilon=1 \sim 80 \varepsilon_{0}} \longrightarrow \mathbf{G}(\mathbf{t})
$$

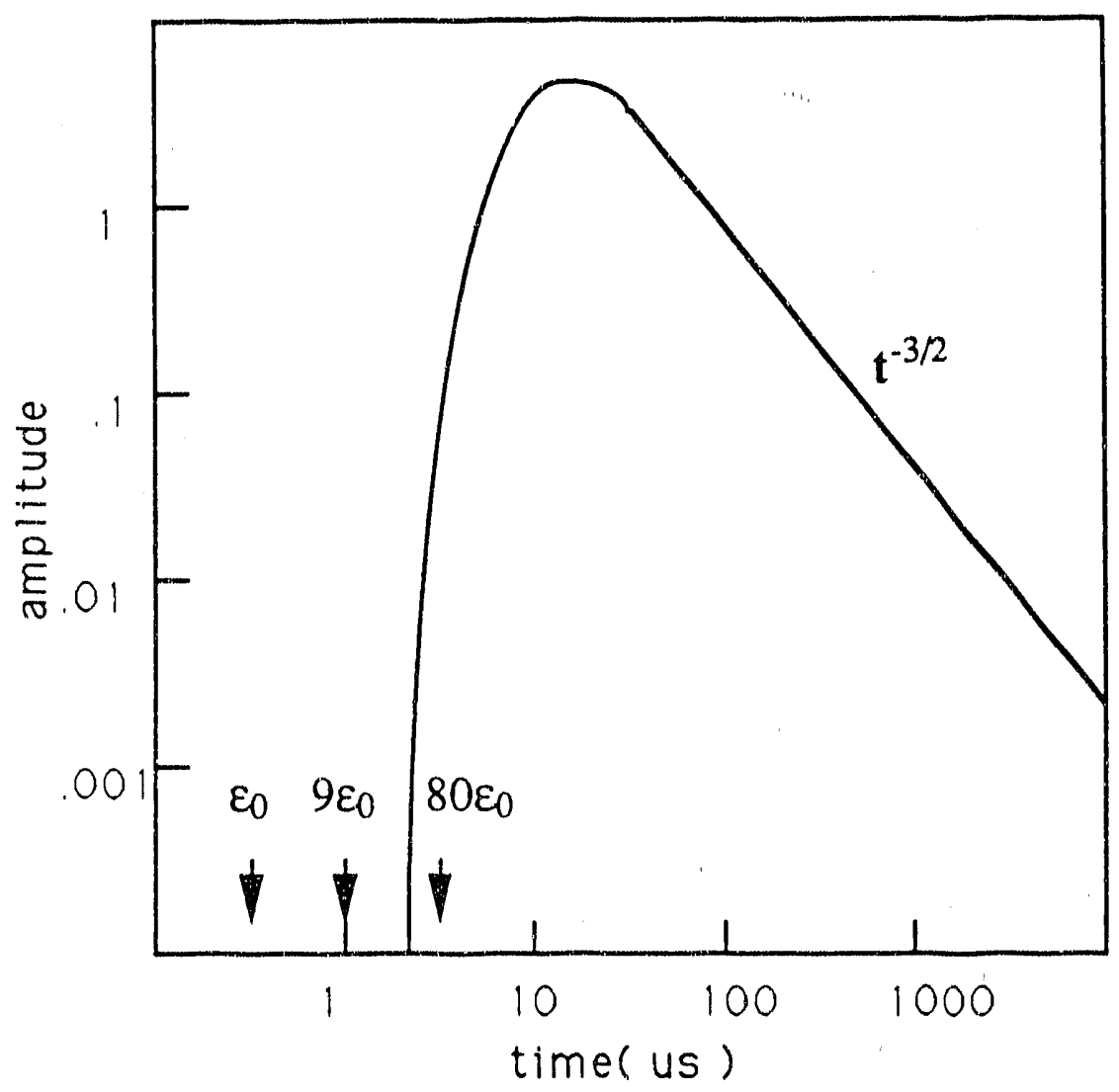

Figure 3-4 Scalar Green's function (diffusion equation) $100 \mathrm{~m}$ away from the source. Arrows indicate the arrival time of wavefront (in the case of wave equation) when $\varepsilon=1,9,80 \varepsilon_{0}$ respectively. 


\section{Initial Time}
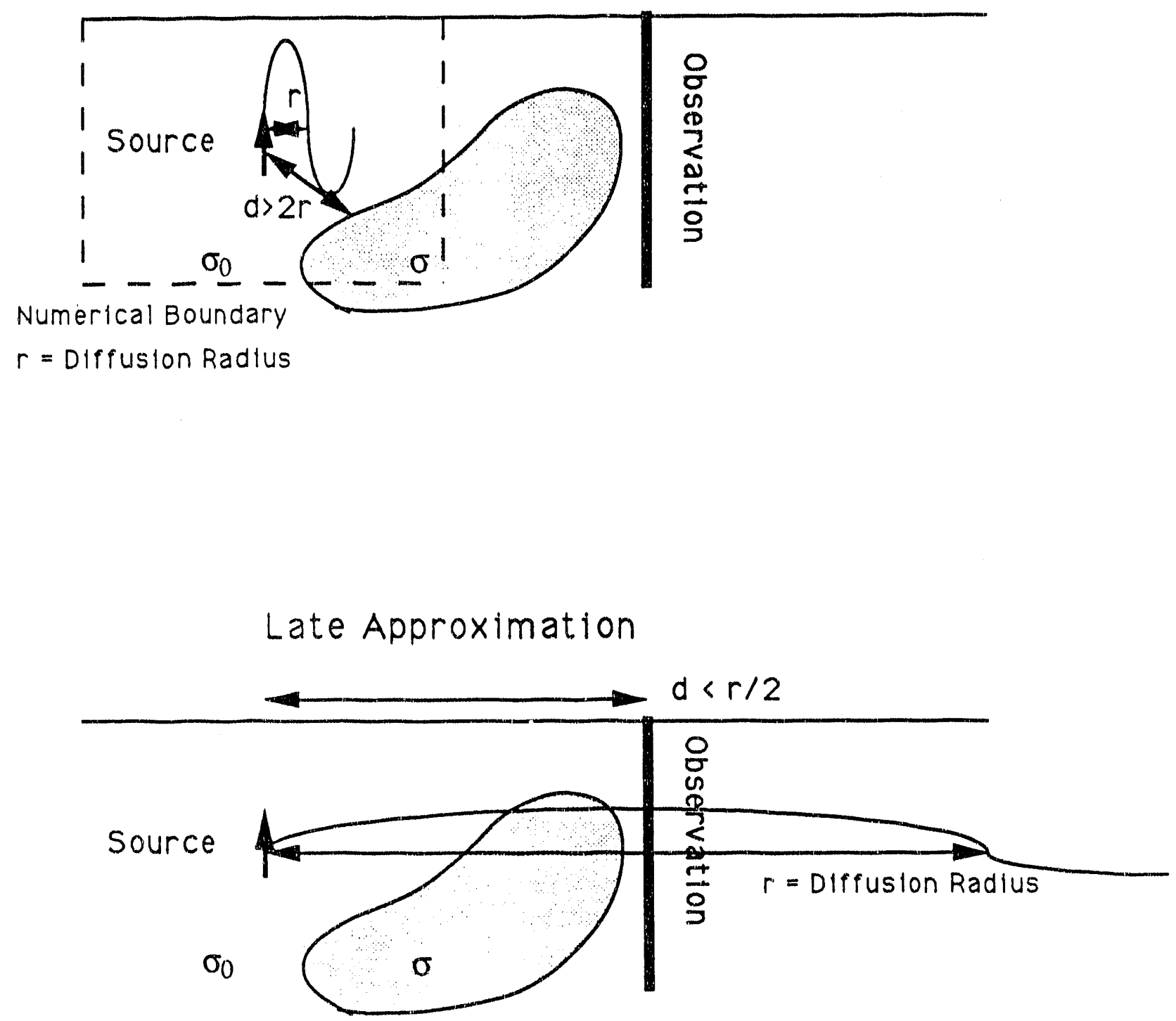

Figure 3-5 Illustration of the latest possible starting time and earliest possible ending time in the numerical calculation. 


\section{Numerical Results}

In this chapter numerical results from this $(t-k)$ algorithm will be compared with existing pseudo-analytic and 3-D integral equation solutions. Here, the pseudo-analytic solution is defined as an analytically formulated solution which requires numerical integration. The range of parameters used in this chapter are $1-100 \Omega m$ resistivity, $1-100 \mathrm{~m}$ region of interest, and $1 \mu \mathrm{sec}$ - 1 msec time intervals. Different geological situations can be scaled up or down from these ranges by the scaling relations

$$
\frac{l_{1}^{2}}{t_{1} \rho_{1}}=\frac{l_{2}^{2}}{t_{2} \rho_{2}}
$$

where $l$ is the distance, $\rho$ is the resistivity, and $t$ is the time (Frischknecht, 1987).

\subsection{Half space}

As the first example, the response of a buried, unit step-current, magnetic dipole in a half space has been calculated and compared with the pseudoanalytic solution. The pseudo-analytic solution is the frequency domain 1-D layered solution (Kaufman and Keller, 1983) transformed to the time domain using a filtering technique (Anderson,1975).

Since the distance from the source to the nearest inhomogeneity $(25 \mathrm{~m}$, from dipole to surface) has been decided in the model, a proper initial time becomes (equation 3.24)

$$
t_{i}=\frac{\mu \sigma}{4}\left(\frac{r}{2}\right)^{2}=0.47 \text { usec }
$$


The next step is to determine the grid-spacing. If the grid-spacing is small, the coverage of wavenumbers will be wide, but, with a finite number of discretizations, low wavenumbers, where the amplitude of the potentials $f$ and $g$ are large, will be poorly represented. The other hand, if the gridspacing is large, the coverage of wavenumbers will be narrow, and truncated amplitudes will be appear in the low wavenumber region as an aliasing effect during the inverse Fourier tranform.

In this numerical exercise, the initial time was set to 0.5 usec and, with $64 \times 64 \times 32$ nodes in $\mathrm{x}, \mathrm{y}$, and $\mathrm{z}$ direction, the grid-spacing was in all directions set to $2.5 \mathrm{~m}$. So the Nyquist wavenumber becomes

$$
k_{n}=\frac{\pi}{\Delta}=0.4 \pi,
$$

and the amplitudes of the potential $f$ which are less than

$$
f\left(k_{n}, t_{i}\right)=\exp \left(-\frac{t_{i}}{\mu \sigma} k_{n}^{2}\right)=0.0018
$$

have been neglected.

When the time reached $10 t_{i}$, the grid-spacing was doubled to $5 \mathrm{~m}$ rather than tripled as shown in the previous chapter. The purpose is to test the flexibility of representing the potentials with a different coverage. With the doubling after the first time decade, the coverage of wavenumbers will be wider than the initial condition and the wavenumber coefficients will be less well represented with the same number of nodes. But this flexibility is aeeded when a realistic 3-D conductivity structure is modelled where each coefficient may decay with a different speed and the coefficients which have been truncated by a large grid-jump will not be appear in the numerical calculation again. 
The number of time-steps and the time-interval at each time-step were gradually increased for each decade. The avarage time-step at early times is one half of that specified by Euler's stability condition and about equal to that at late times.

The major portion of the floating-point operations (FLOPs) in this alogrithm is used in evaluating the 3-D forward and inverse fast Fourier transforms (FFT). Since 3-D FFT requires

$$
\ln n\left(\log _{2} l+\log _{2} m+\log _{2} n\right)
$$

FLOPs for $(l, m, n)$ nodes, about 30 Mega FLOPs are made at each time step and the total calculation takes about $: 0 \mathrm{CPU}$ minutes on a Cray-2 with 1 parallel processor.

Figure (4.2) shows snapshots of the tangential electric fields ("Smoke Ring") in the subsurface along with whole space responses. The overall responses are very similar except at the air-earth interface where the diffusion fields have to satisfy both the continuity of tangential electric field and the infinite diffusion velocity at the air. Figure (4.3) shows the results of these conditions in linear time and amplitude scales. It clearly shows that the fields move faster than in the subsurface but not as fast as can one expect from the infinite velocity of the air. Figure (4.4) shows the comparision of the numerical solution with the 1-D pseudo-analytic solution. The following table summarizes the numerical parameters used for this example. 


\begin{tabular}{|c|c|c|c|}
\hline time $[\mu \mathrm{sec}]$ & $.5-5$ & $5-50$ & $50-500$ \\
\hline grid-spacing $[\mathrm{m}]$ & $2.5,2.5,2.5$ & $5,5,5$ & $10,10,10$ \\
\hline no. of nodes & $64,64,32$ & $64,64,32$ & $64,64,32$ \\
\hline numerical domain $[\mathrm{m}]$ & $\pm 80,80,40$ & $\pm 160,160,80$ & $\pm 320,320,160$ \\
\hline no. of time-steps & 300 & 500 & 1000 \\
\hline Euler's stability $\delta t[\mu \mathrm{sec}]$ & 0.027 & 0.11 & 0.44 \\
\hline average $\delta t$ used $[\mu \mathrm{sec}]$ & 0.015 & 0.09 & 0.45 \\
\hline
\end{tabular}


Whole Space

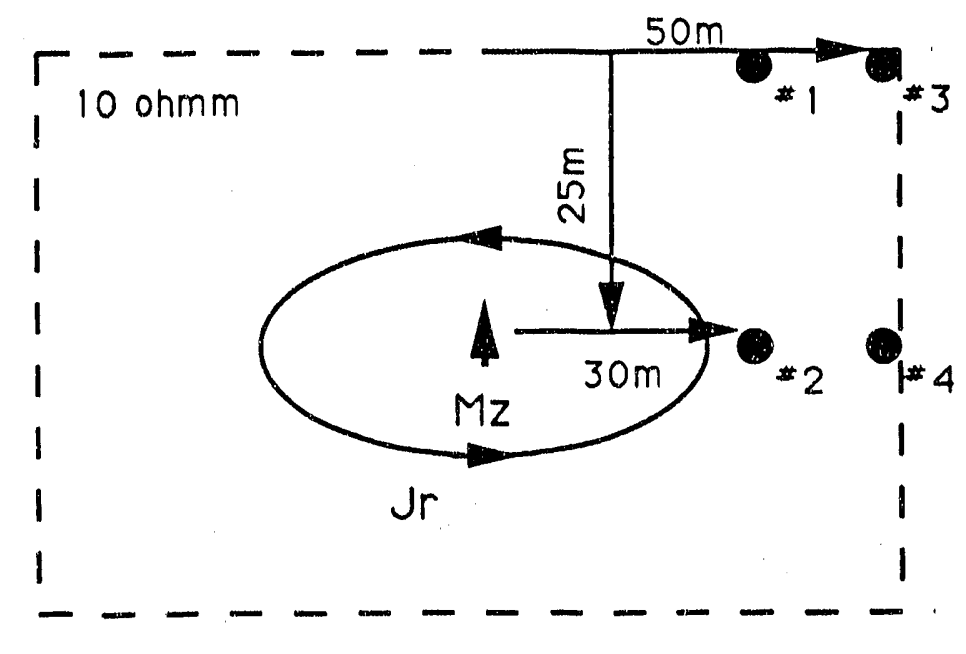

Half Space

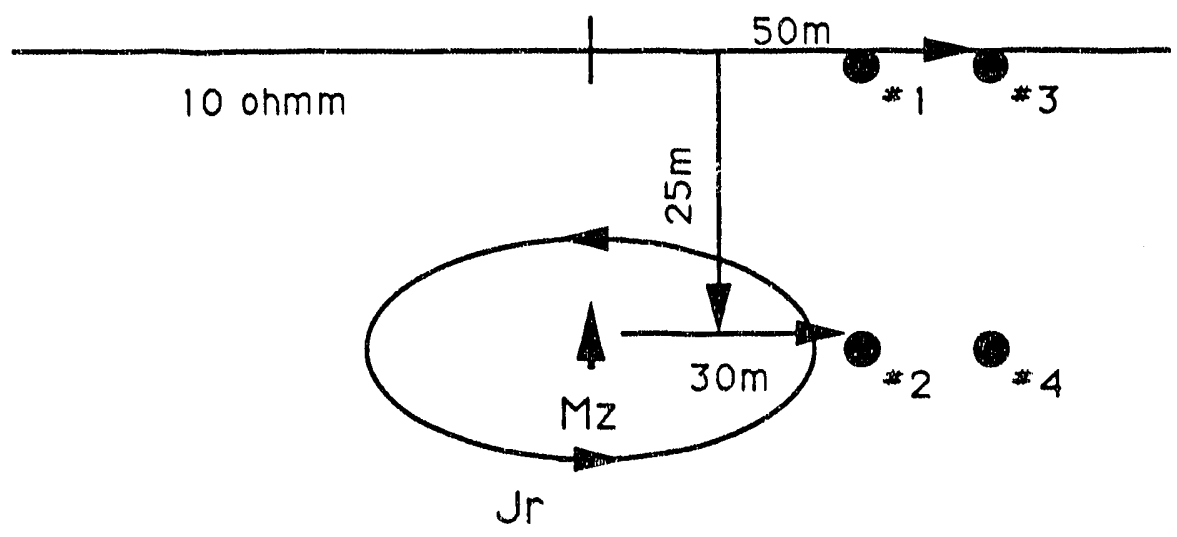

Figure 4-1 Whole and half space model. The source is at the center for the whole space and $25 \mathrm{~m}$ below surface for the half space. 

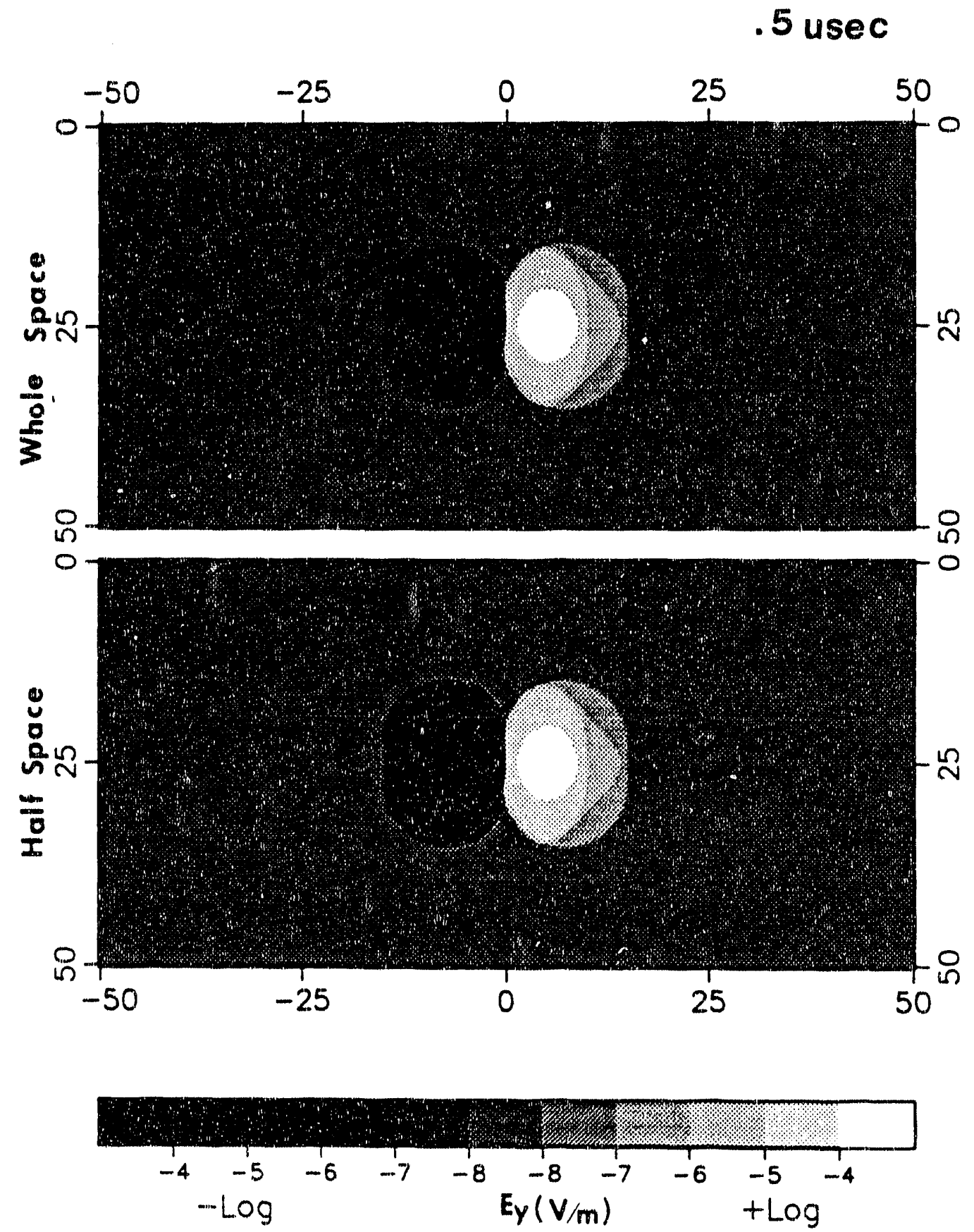

Figure 4.2 (a)Snapshots of the tangential electric field (Ey) $(t=0.5$ usec). 

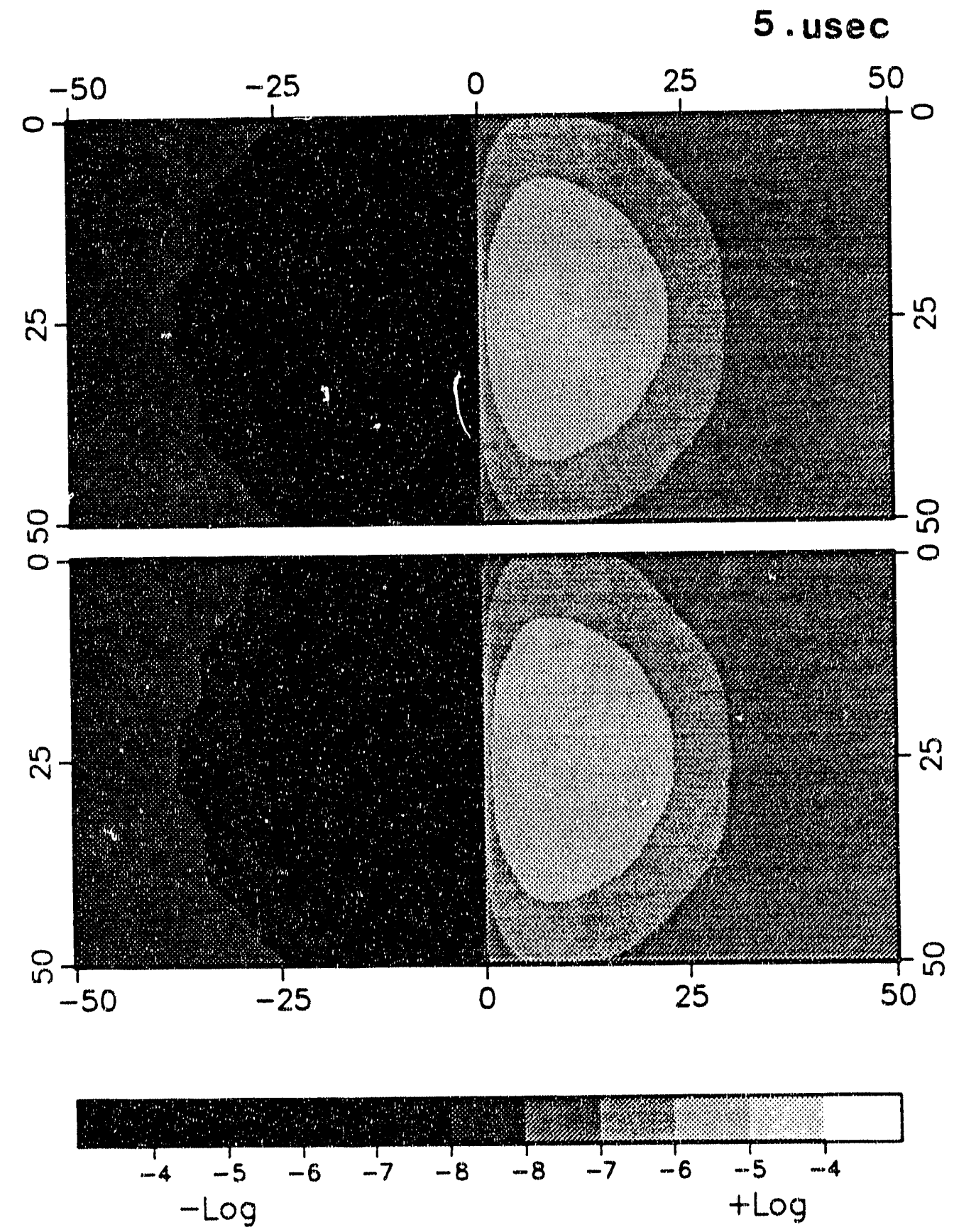

Figure 4-2 (b)Snapshots of Ey( $t=5$. usec). 
10usec
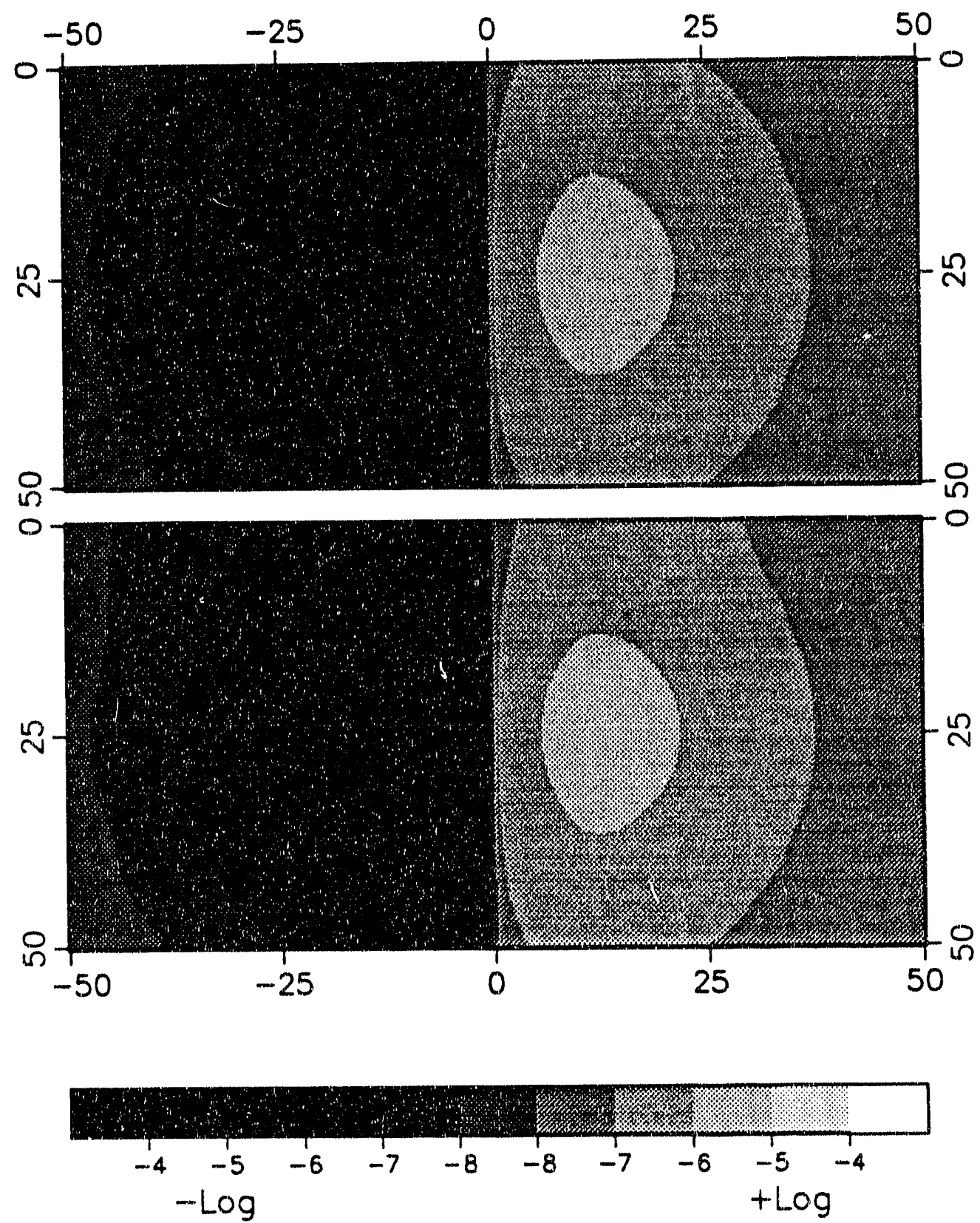

Figure 4-2 (c)Snapshots of $E y(t=10$. usec). 


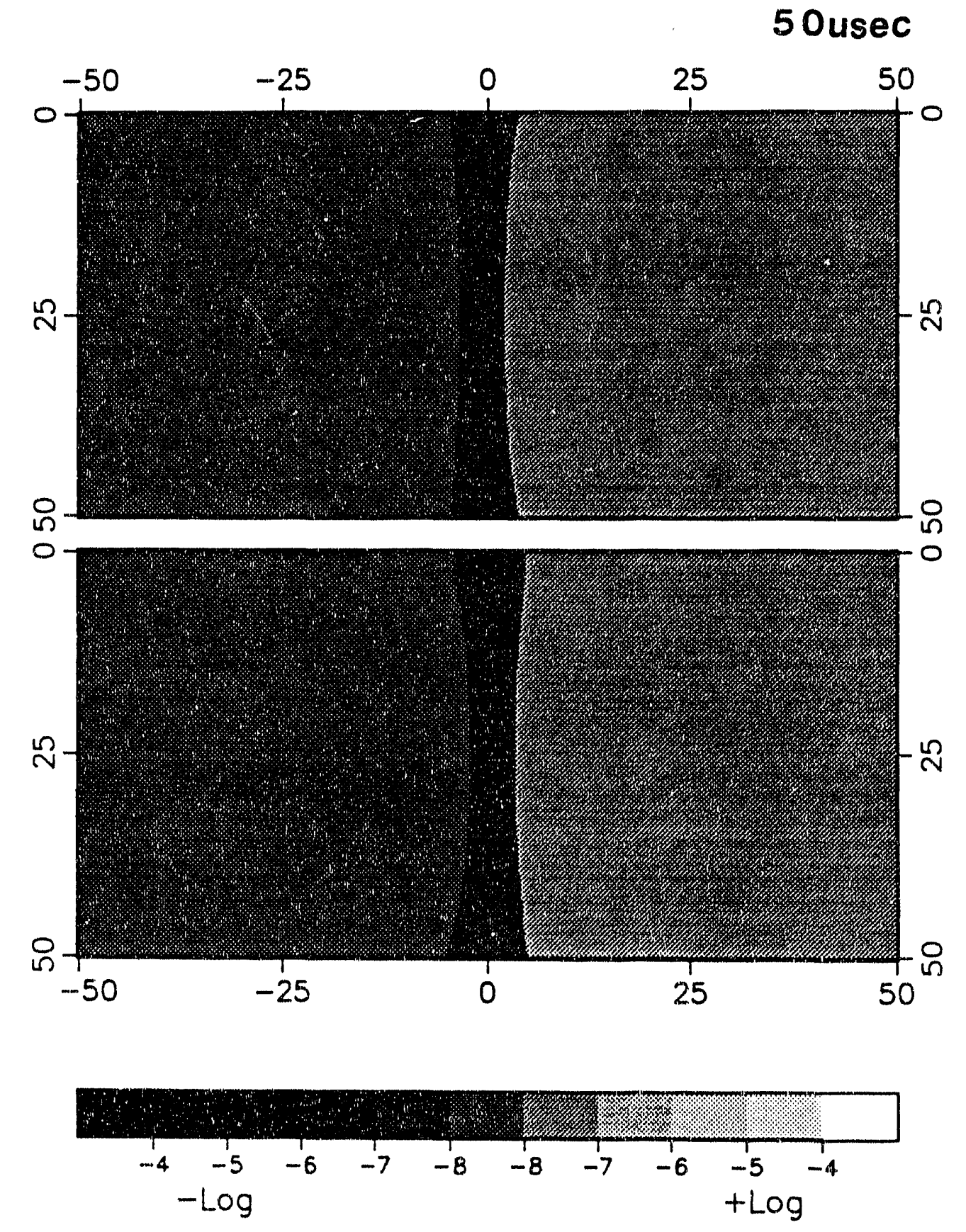

Figure 4-2 (d)Snapshots of $E_{y^{\prime}}(t=50$. usec). 

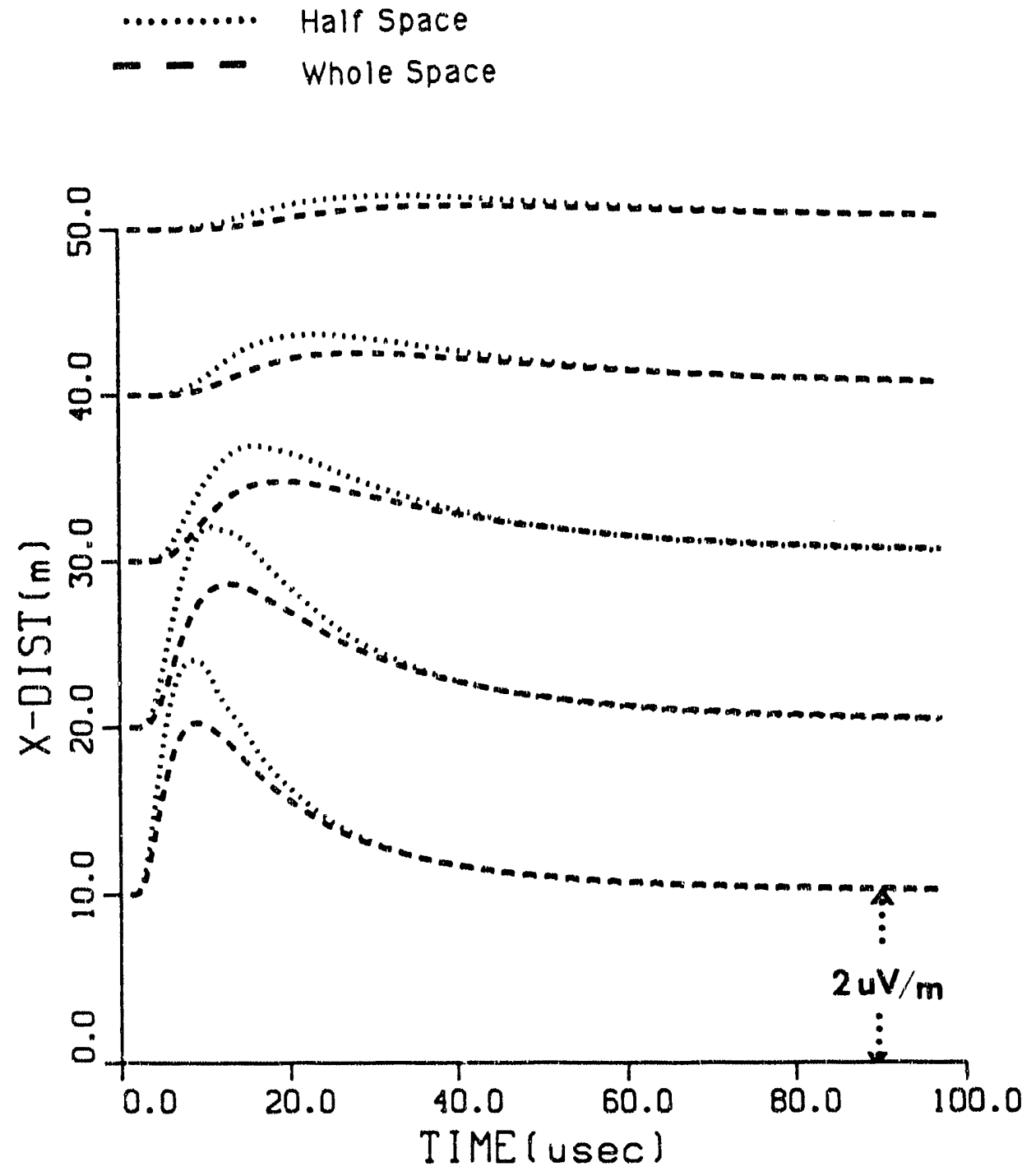

Figure 4-3 Time traces of Ey at the surface. Each trace has shifted by $2 \mathrm{uV} / \mathrm{m}$ (linear amplitude and time scales). 
\begin{tabular}{ll}
- & Pseudo-Analytic Half Space \\
\hline & Numerical Half Space \\
$\Delta$ & Whole Space
\end{tabular}
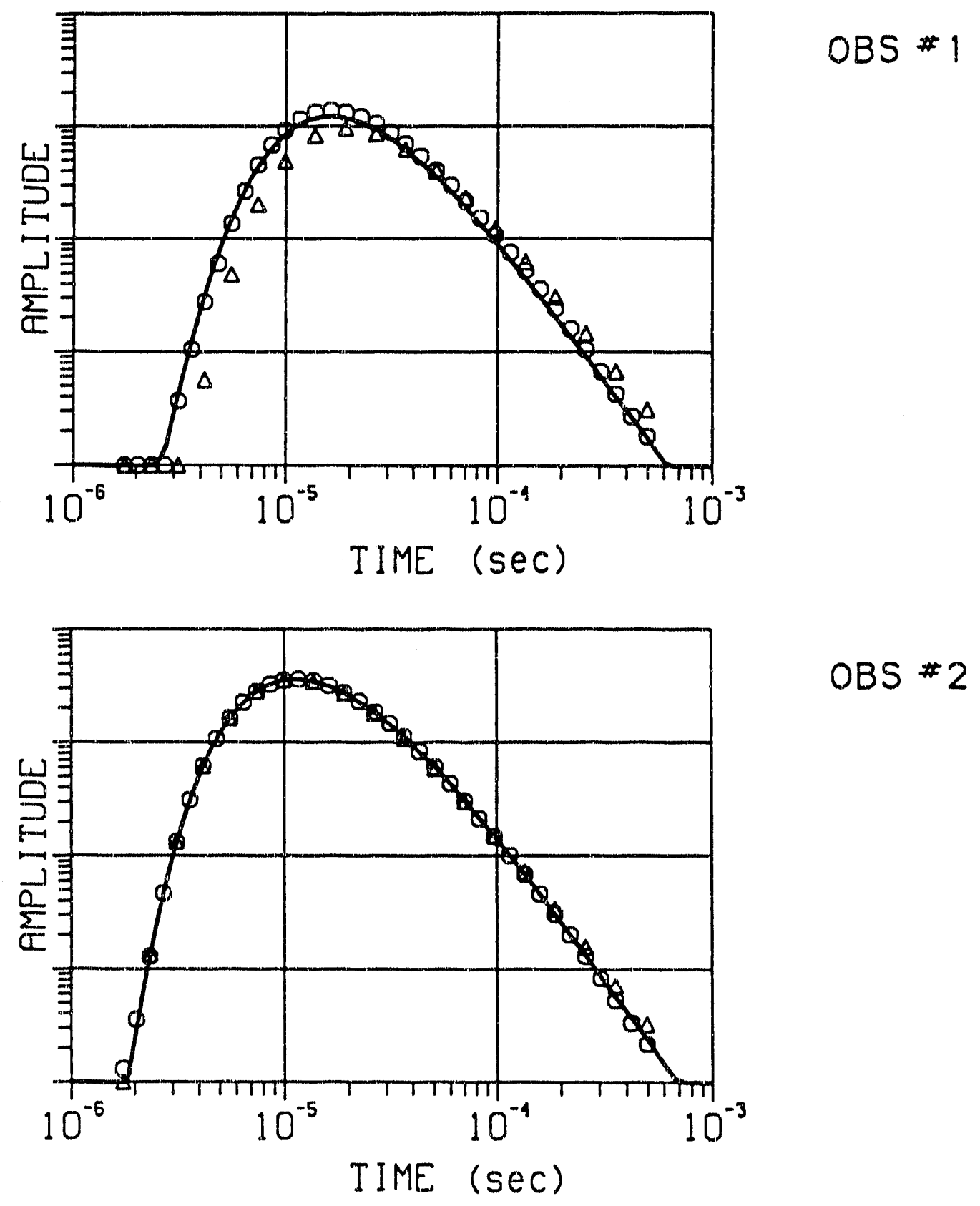

Figure 4-4 (a)Comparisons with 1-D pseudo-analytic solution at $(30,0,0)$ and $(30,0,25)$. 

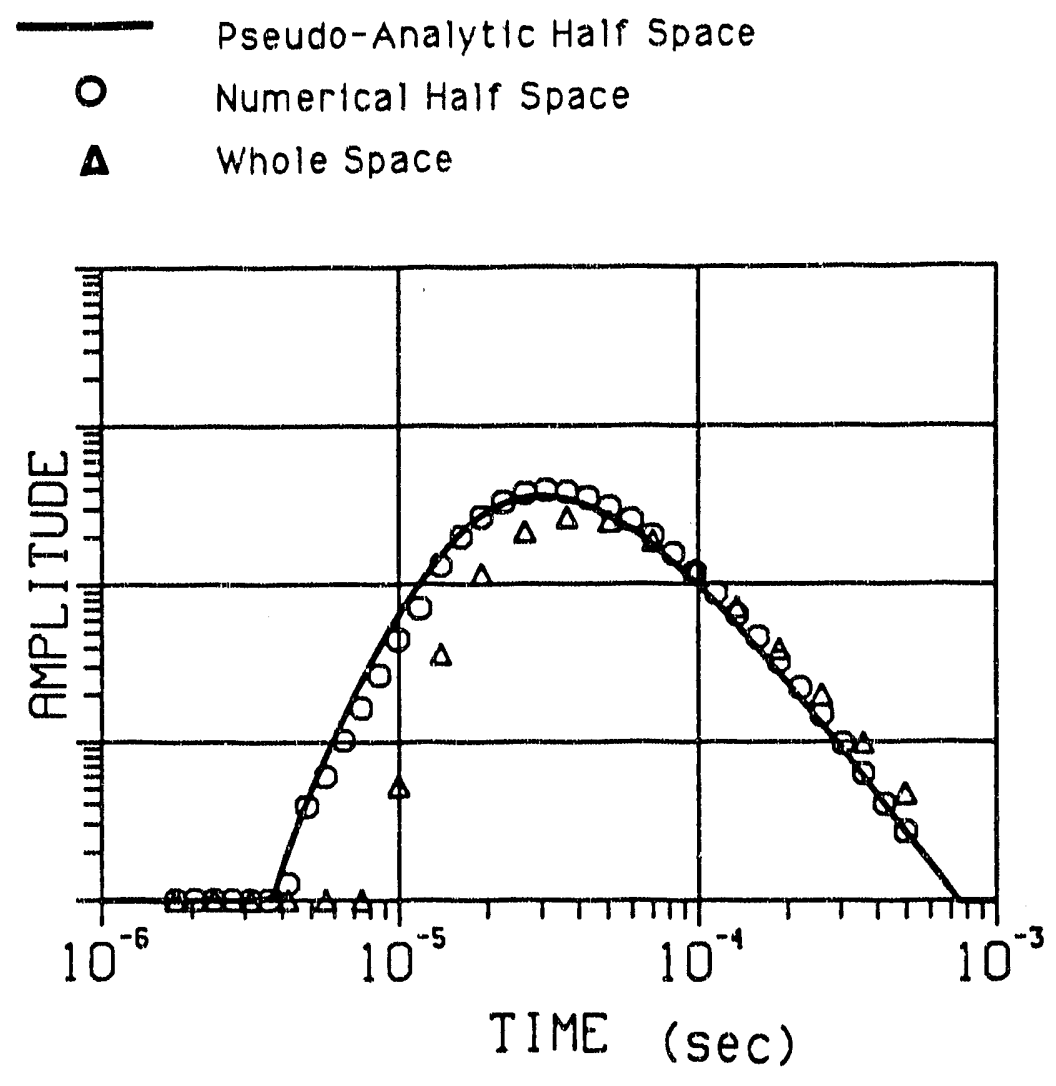

OBS $\# 3$

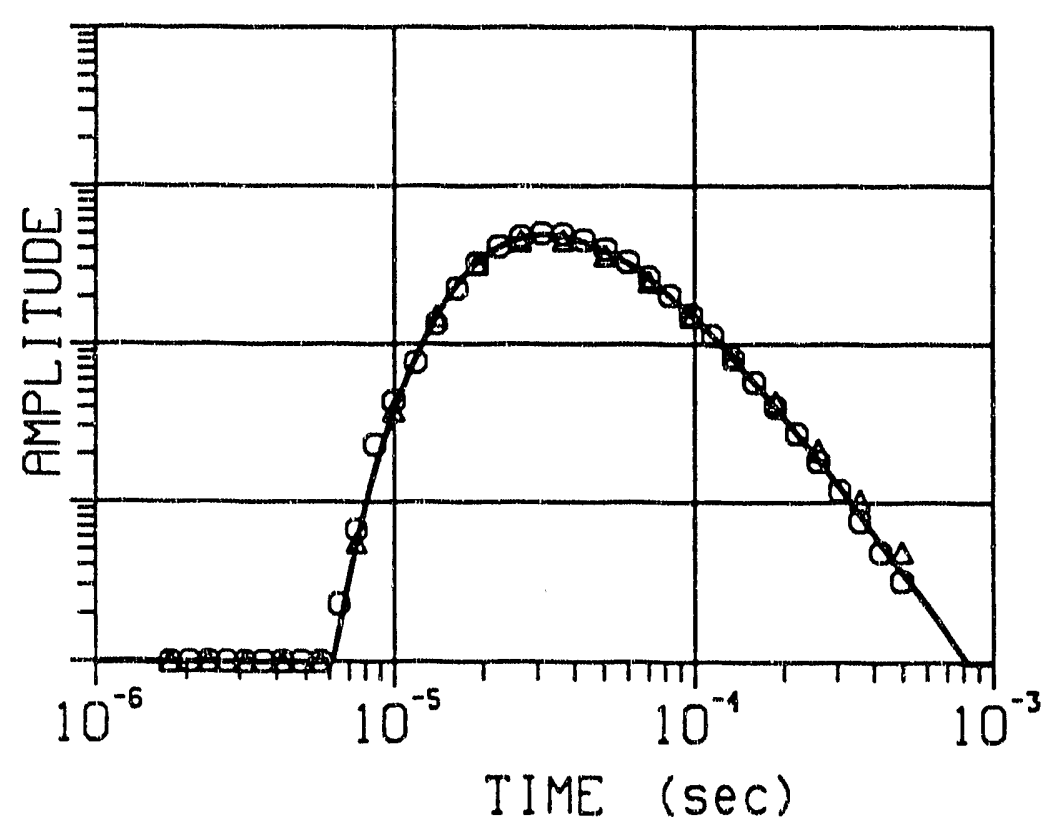

OBS \#4

Figure 4-4 (b)Comparisons with 1-D pseudo-analytic solution at $(50,0,0)$ and $(50,0,25)$. 


\subsection{Block in a layered half space}

The response from a buried cube $(10 \times 10 \times 10 \mathrm{~m}, 10 \Omega \mathrm{m})$ in a layered half space due to a buried, unit step-current, magnetic dipole source is shown next. Since the distance from the source to the nearest layer boundary $(15 \mathrm{~m})$ is closer and the background resistivity $(50 \Omega \mathrm{m})$ is higher than in the previous example, the initial time was advanced to $t_{i}=0.1 \mu \mathrm{sec}$ and a smaller gridspacing $(1.25 \times 1.25 \times 2.5)$ was used.

The number of nodes was of the same as in the previous example $(64 \times 64 \times 32)$ except, during the first decade of computation the number of nodes in the inverse FFT which evaluates $E(\vec{k}, t)$ from $E(\vec{r}, t)$ was doubled in order to evaluate high wavenumber coefficients more accurately and prevent aliasing effects during the Fourier transforms. As a result, the total computation took twice the CPU time of the previous example.

Figure (4.6) shows the snapshots of the tangential electric fields and Figures (4.7) and (4.8) show the profile of the tangential electric and vertical magnetic fieids $30 \mathrm{~m}$ away from the source. As shown in these figures, unlike wave propagation where one can expect strong scattering from inhomogeneous boundaries, the diffusion field behaves very smoothly for the inhomogeneous medium and the response from the 3-D block quickly disappears at late times or away from the block.

The response of the layered half space without the block has been calculated and compared with the 1-D pseudo-analytic solution. The secondary 3-D response which is the difference between the 3-D and 1-D responses has been compared with $3-\mathrm{D}$ integral equation solutions. 
The impulse current response vertical magnetic field is directly caluculated from the step-current electric field. From the Maxwell's equation (2.2),

$$
\int_{l} E(\vec{r}, t) d l=-\mu_{0} \frac{\partial}{\partial t} \int_{S} H(\vec{r}, t) d S .
$$

Using the Simpson's rule for the line integration in the finite difference formulation,

$$
\begin{gathered}
\frac{\partial}{\partial t} H_{z}^{i, j, k}=\frac{-1}{\mu_{0} \Delta x \Delta y} \\
{\left[\frac{\Delta y}{4}\left(E_{y}^{i-1, j+1, k}+3 E_{y}^{i, j+1, k}+E_{y}^{i+1, j+1, k}-E_{y}^{i-1, j-1, k}-3 E_{y}^{i, j-1, k}-E_{y}^{i-1, j+1, k}\right)\right.} \\
\left.+\frac{\Delta x}{4}\left(E_{x}^{i-1, j-1, k}+3 E_{x}^{i, j-1, k}+E_{x}^{i+1, j-1, k}-E_{x}^{i-1, j+1, k}-3 E_{x}^{i, j+1, k}-E_{x}^{i+1, j+1, k}\right)\right]
\end{gathered}
$$

As shown in Figure (4.9) to (4.11), the electric fields agree well with 1-D solution, whereas the vertical magnetic fields lag the 1-D solution when the fields change rapidly. This comes from the fact that the finite differences of the electric fields cannot follow the true derivatives when the fields change rapidly. It can be further corrected by direct evaluation from the potential $f$ and $g$.

The following table summarizes the numerical parameters used for this example.

\begin{tabular}{|c|c|c|c|}
\hline time $[\mu \mathrm{sec}]$ & $.1-1$ & $1-10$ & $10-100$ \\
\hline grid-spacing $[\mathrm{m}]$ & $1.25,1.25,2.5$ & $2.5,2.5,5$ & $5,5,5$ \\
\hline no. of nodes & $64,64,32$ & $64,64,32$ & $64,64,32$ \\
\hline numerical domain $[\mathrm{m}]$ & $\pm 40,40,40$ & $\pm 80,80,80$ & $\pm 160,160,80$ \\
\hline no. of time-steps & 500 & 500 & 500 \\
\hline Euler's stability $\delta t[\mu \mathrm{sec}]$ & 0.0018 & 0.005 & 0.021 \\
\hline average $\delta t$ used $[\mu \mathrm{sec}]$ & 0.0018 & 0.018 & 0.18 \\
\hline
\end{tabular}


3-D BLOCK in LAYERED HALF SPACE

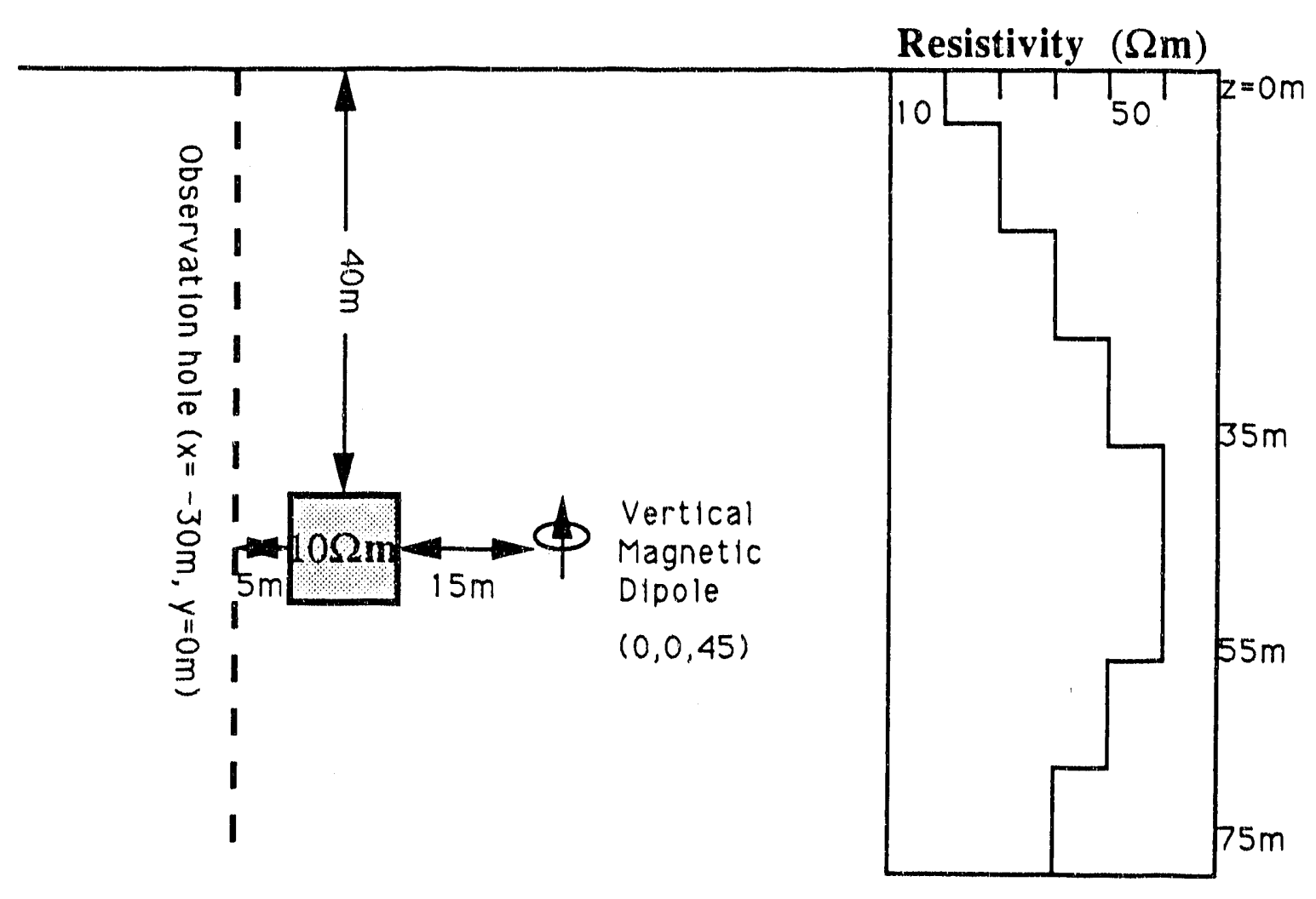

Figure 4.5 3-D model. A cube $(10 \times 10 \times 10 \mathrm{~m})$ is at $40 \mathrm{~m}$ below surface and $15 \mathrm{~m}$ away from the source. The righthand side diagram shows the resistivity of the model layers. 

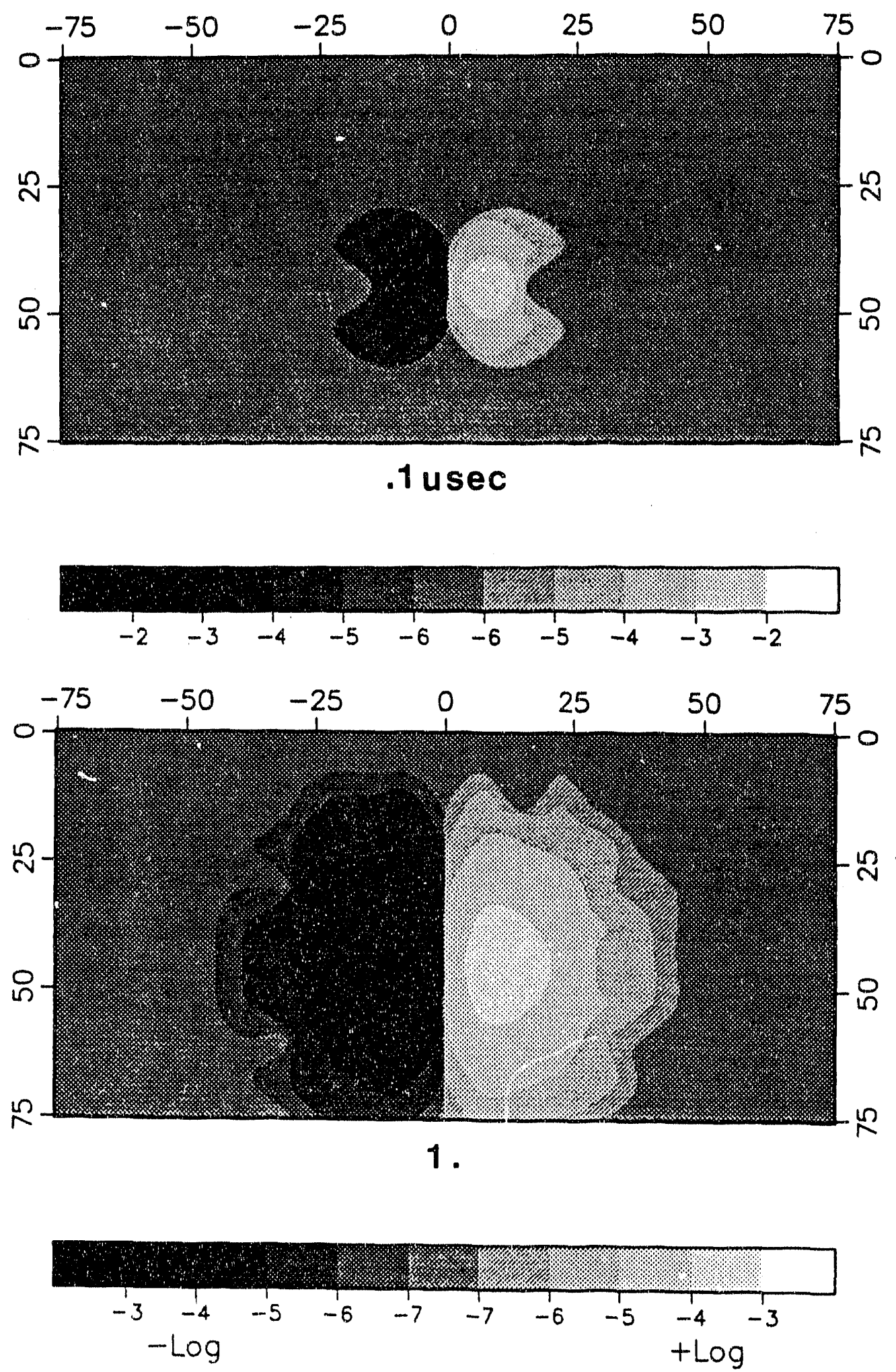

Figure 4-6 (a) Snapshots of Ey at .1 and 1 usec. 

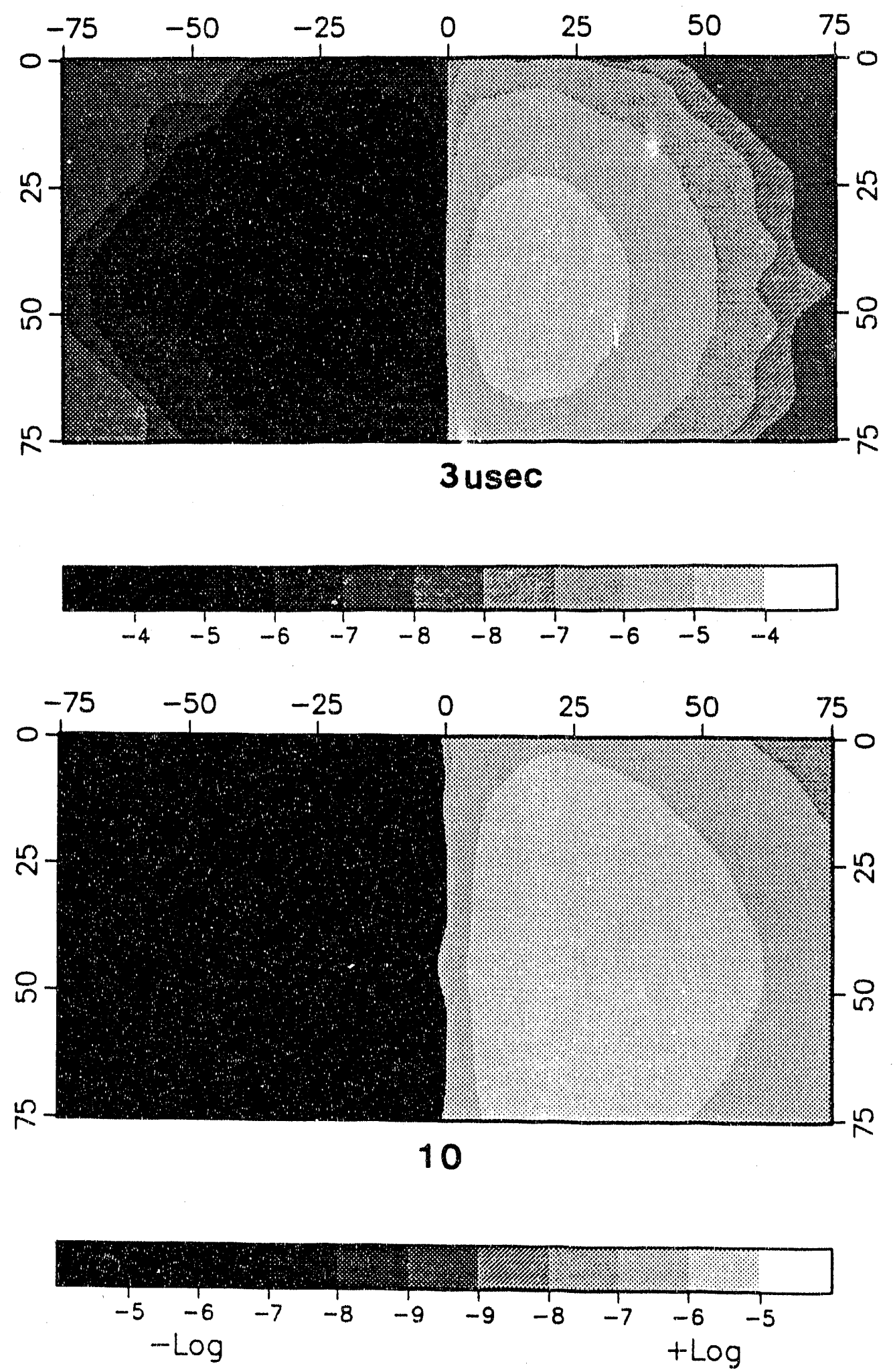

Figure 4-6 (b) Snapshots of Ey at 3 and 10 usec. 


$$
\begin{aligned}
& - \text { - } \\
& \text {-......... T-K Layered Half Space } \\
& \text { T-K Wlth 3-D Block }
\end{aligned}
$$

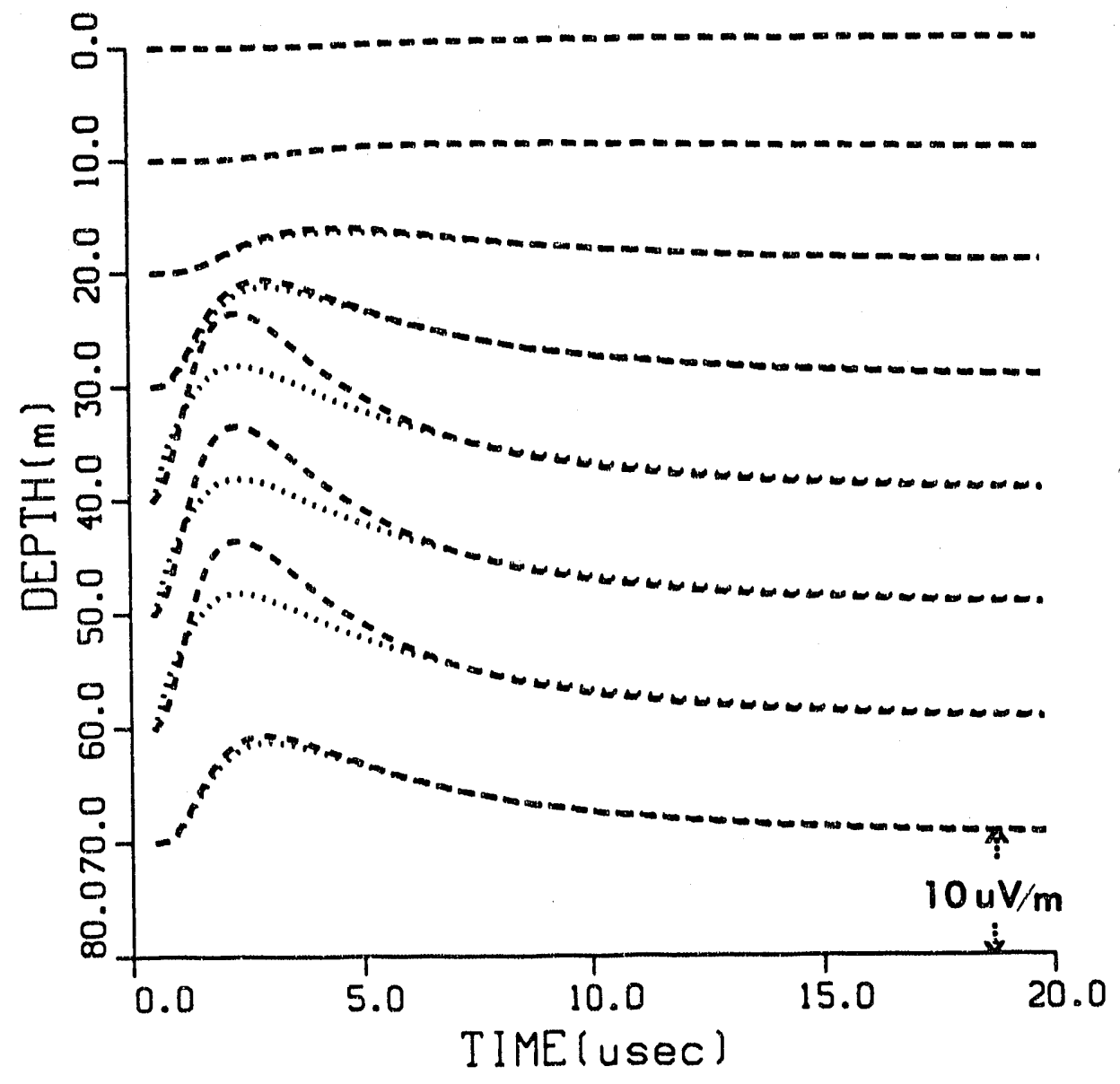

Figure 4-7 Time traces of Ey at the vertical observation hole with or without the block. Each trace has shifted by $10 \mathrm{uV} / \mathrm{m}$ (linear amplitude and time scales). 
- - T-K Layered Half Space

........... T-K With 3-D Block

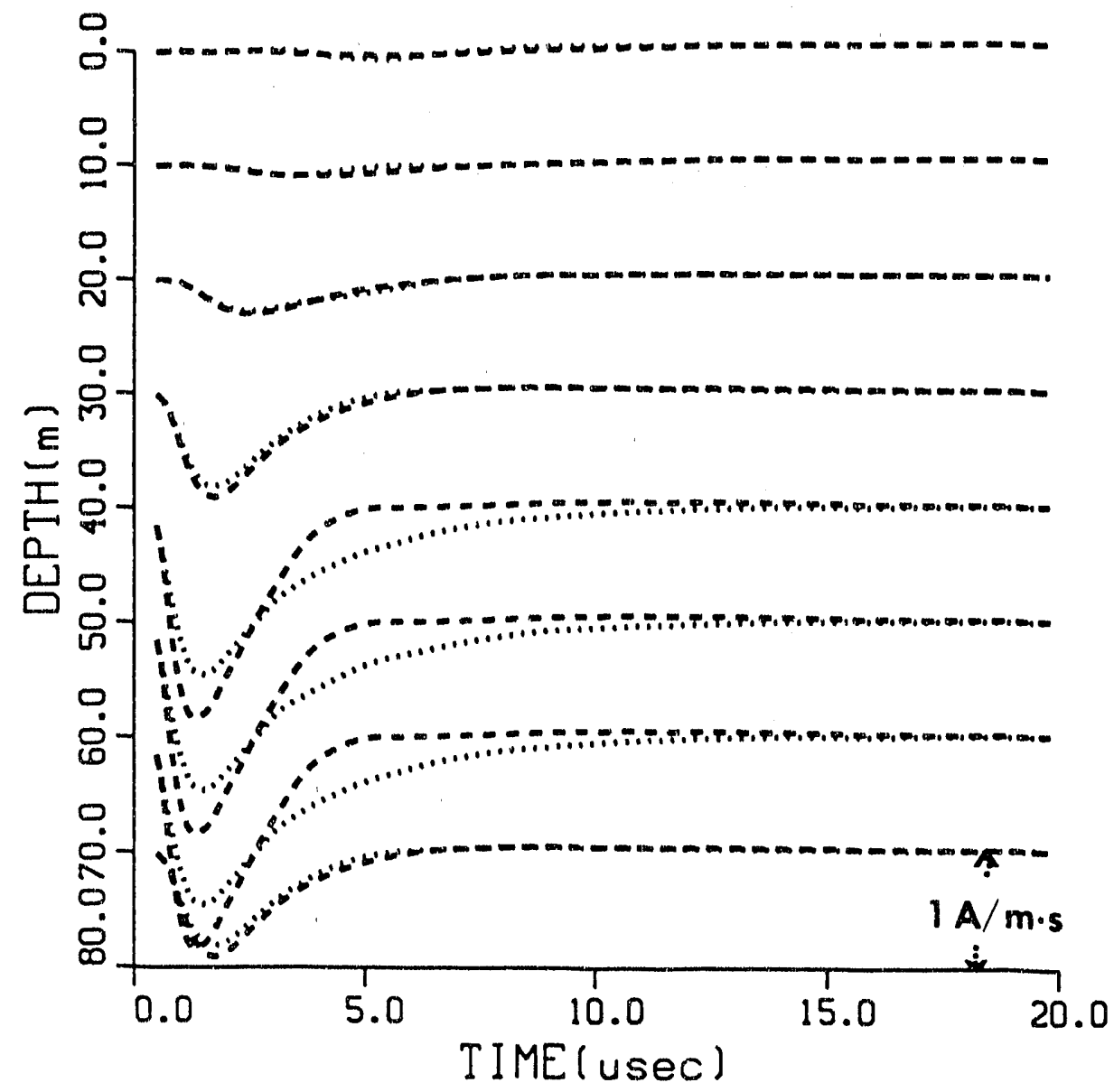

Figure 4-8 Time traces of vertical magnetic field at the vertical observation hole with or without the block. Each trace has shifted by $1 \mathrm{~A} / \mathrm{m} / \mathrm{s}$ (linear amplitude and time scales). 

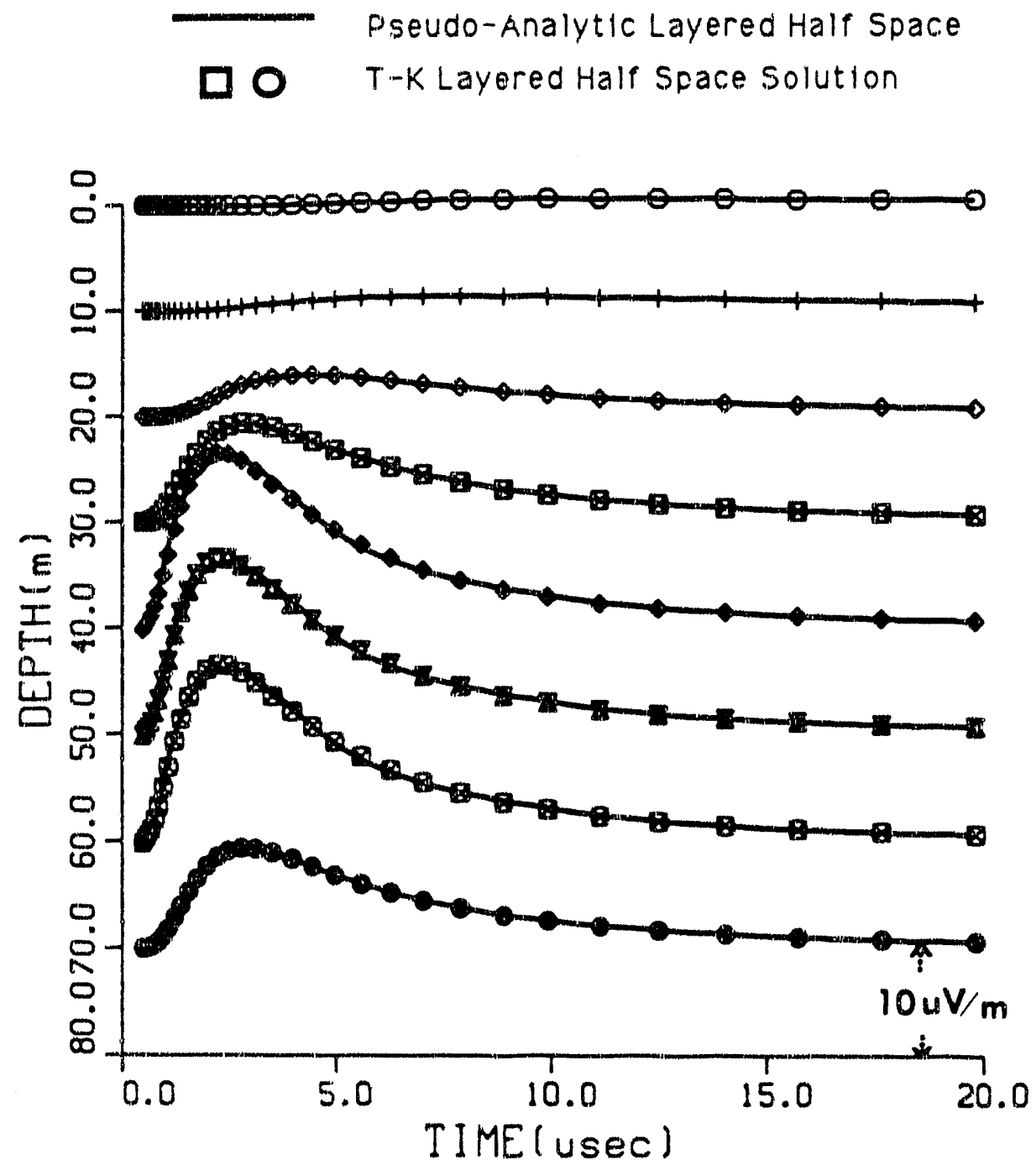

Figure 4-9 Comparisons of the tangential electric field for the layer model with the 1-D pseudo-analytis solution along the observation hole . 

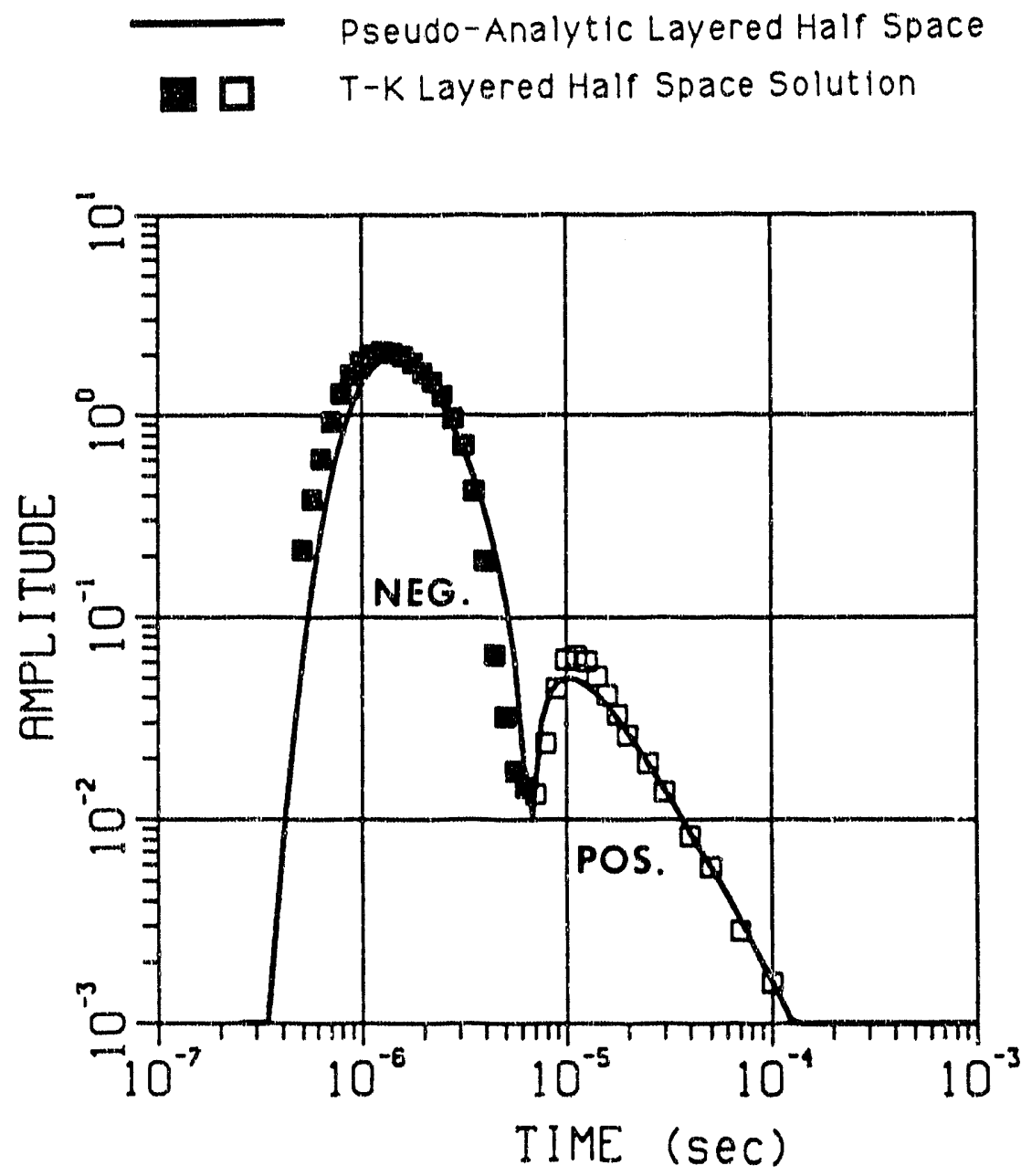

Figure 4-10 Comparisons of the vertical magnetic field for the layer model with the 1-D pseudo-analytic solution $(-30,0,40)$. 

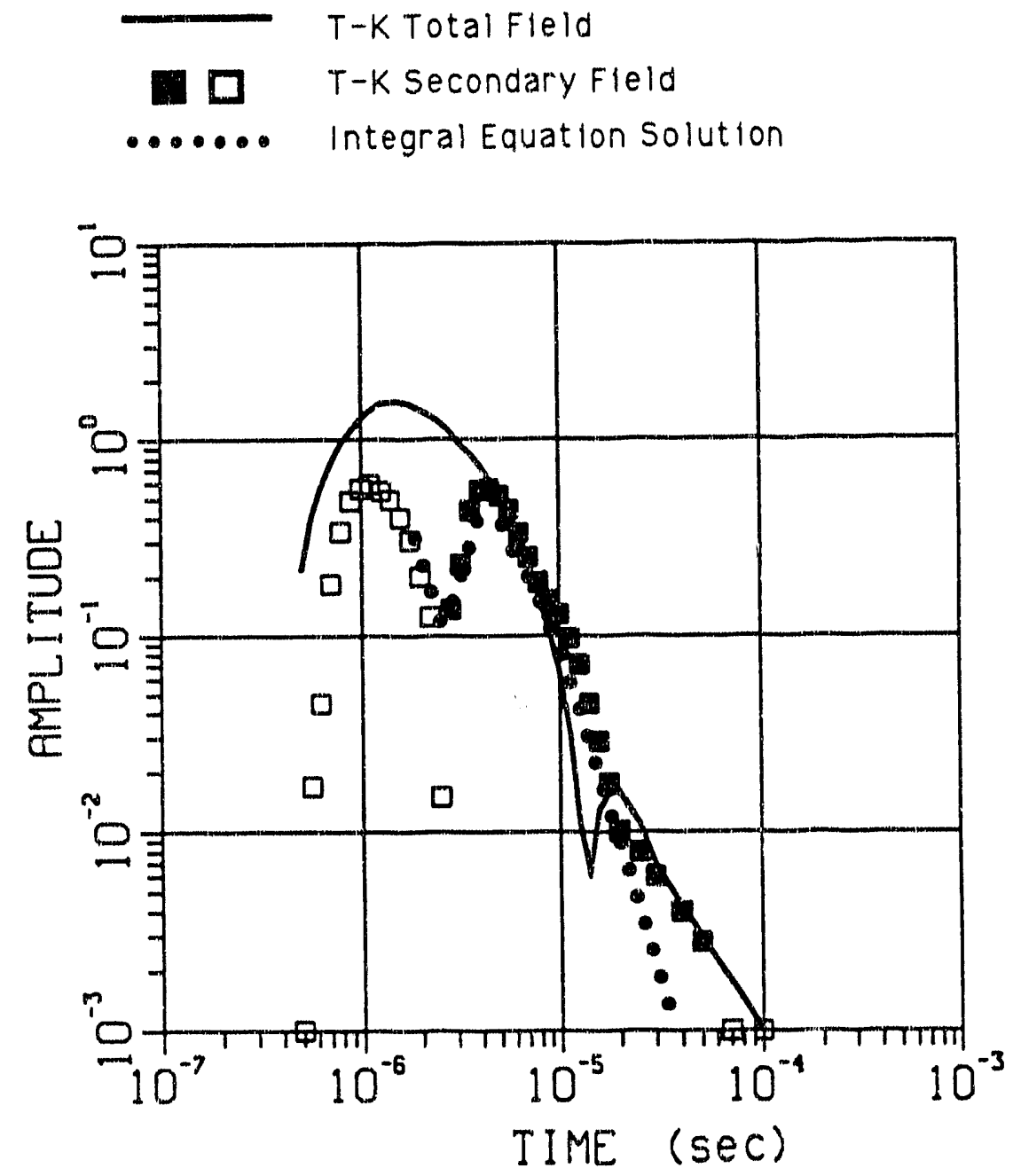

Figure 4-11 Comparisons of the vertical magnetic field for the 3-D model with the 3-D integral equation solution $(-30,0,40)$ (2ndary $\mathrm{H}_{\mathrm{Z}}=3-\mathrm{D} \mathrm{H}_{\mathrm{Z}}-1-\mathrm{D} \mathrm{H}_{\mathrm{Z}}$ ). 


\subsection{Buried sphere}

The response from a buried sphere in a half space by a surface, stepcurrent loop source (Figure 4.12) has been computed and compared with another pseudo-analytic solution (Lee, 1975). Using the symmetry of the model, the computation involves only the positive quadrant of the model. This can be done simply by taking cosine and sine transform in $\mathrm{x}$ and $\mathrm{y}$ for $E_{x}$ and sine and cosine transform in those coordinates for $E_{y}$.

Figure (4.14) shows the tangential electric field at the location of the loop which can be observed in practice by measuring the voltage in the loop after the step-current is turned off. The response from the sphere is surprisingly small, but the result can be understood from the snapshots (Figure 4.13) where it is shown that the major portion of the primary electric field never passes through the sphere, and so the secondary field which is proportional to the primary field would be small.

The following table summarizes the numerical parameters used for this example.

\begin{tabular}{|c|c|c|c|}
\hline time $[\mu \mathrm{sec}]$ & $.5-5$ & $5-50$ & $50-1000$ \\
\hline grid-spacing $[\mathrm{m}]$ & $5,5,5$ & $10,10,10$ & $20,20,20$ \\
\hline no. of nodes & $64,64,32$ & $64,64,32$ & $64,64,32$ \\
\hline numerical domain $[\mathrm{m}]$ & $320,320,160$ & $640,640,320$ & $1.28,1.28,64 \times 10^{3}$ \\
\hline no. of time-steps & 500 & 500 & 1000 \\
\hline Euler's stability $\delta t[\mu \mathrm{sec}]$ & 0.011 & 0.044 & 0.17 \\
\hline average $\delta t$ used $[\mu \mathrm{sec}]$ & 0.009 & 0.09 & 0.9 \\
\hline
\end{tabular}




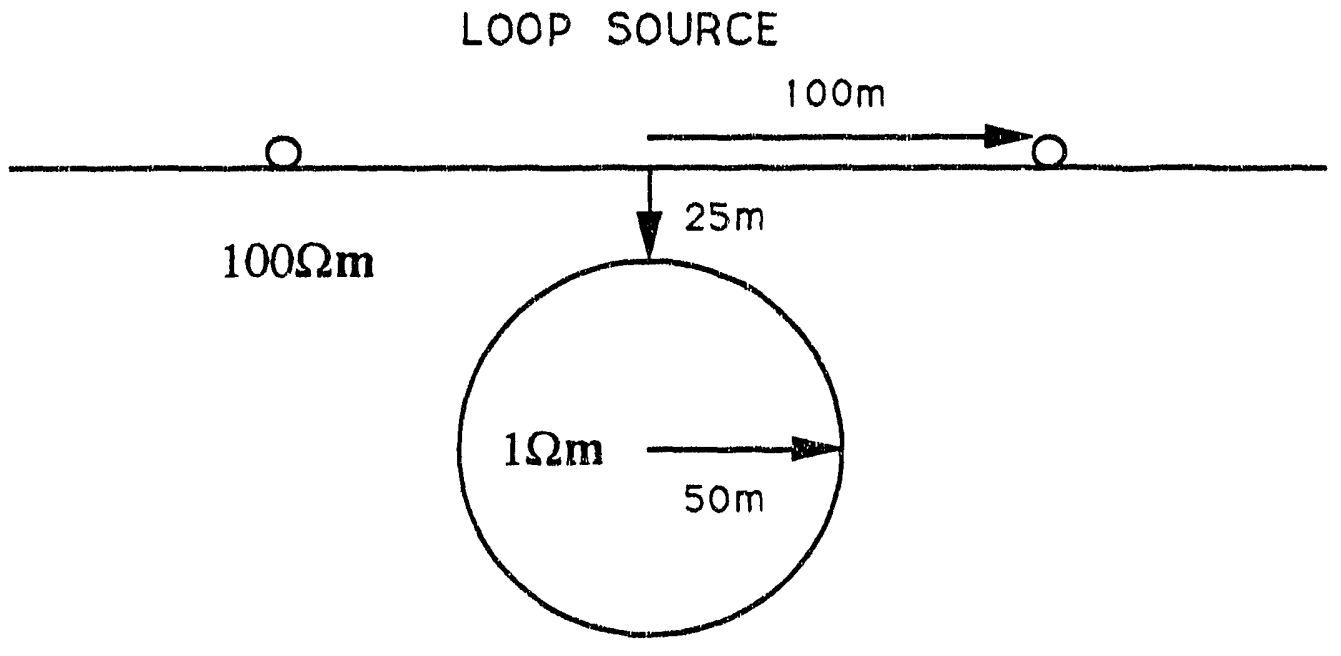

1 usec
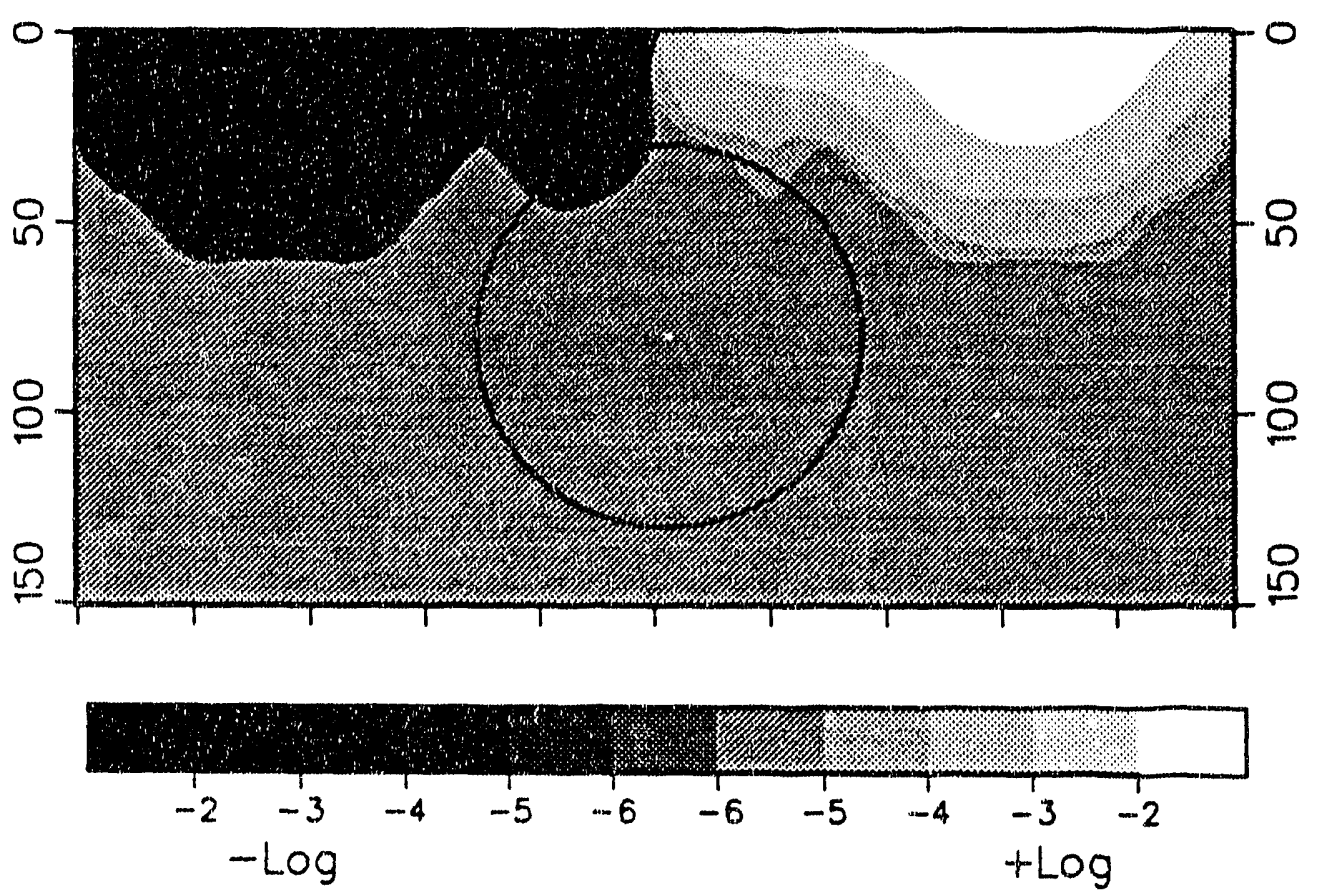

Figure 4-12 Buried sphere model and the snapshot of Ey at an early time (1 usec). 


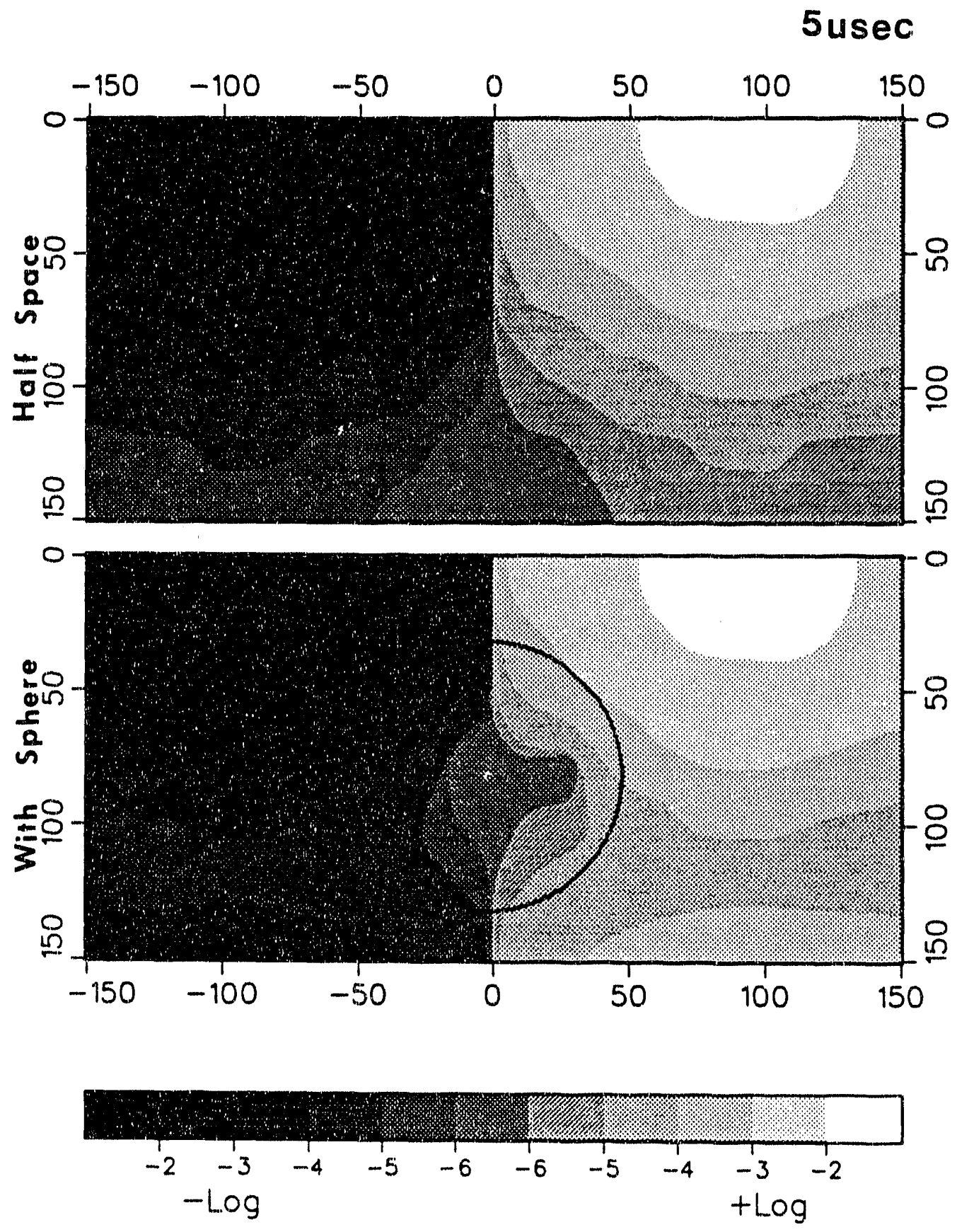

Figure 4-13 (a) Snapshots of half space and with sphere model at $t=5$ usec (Ey). 

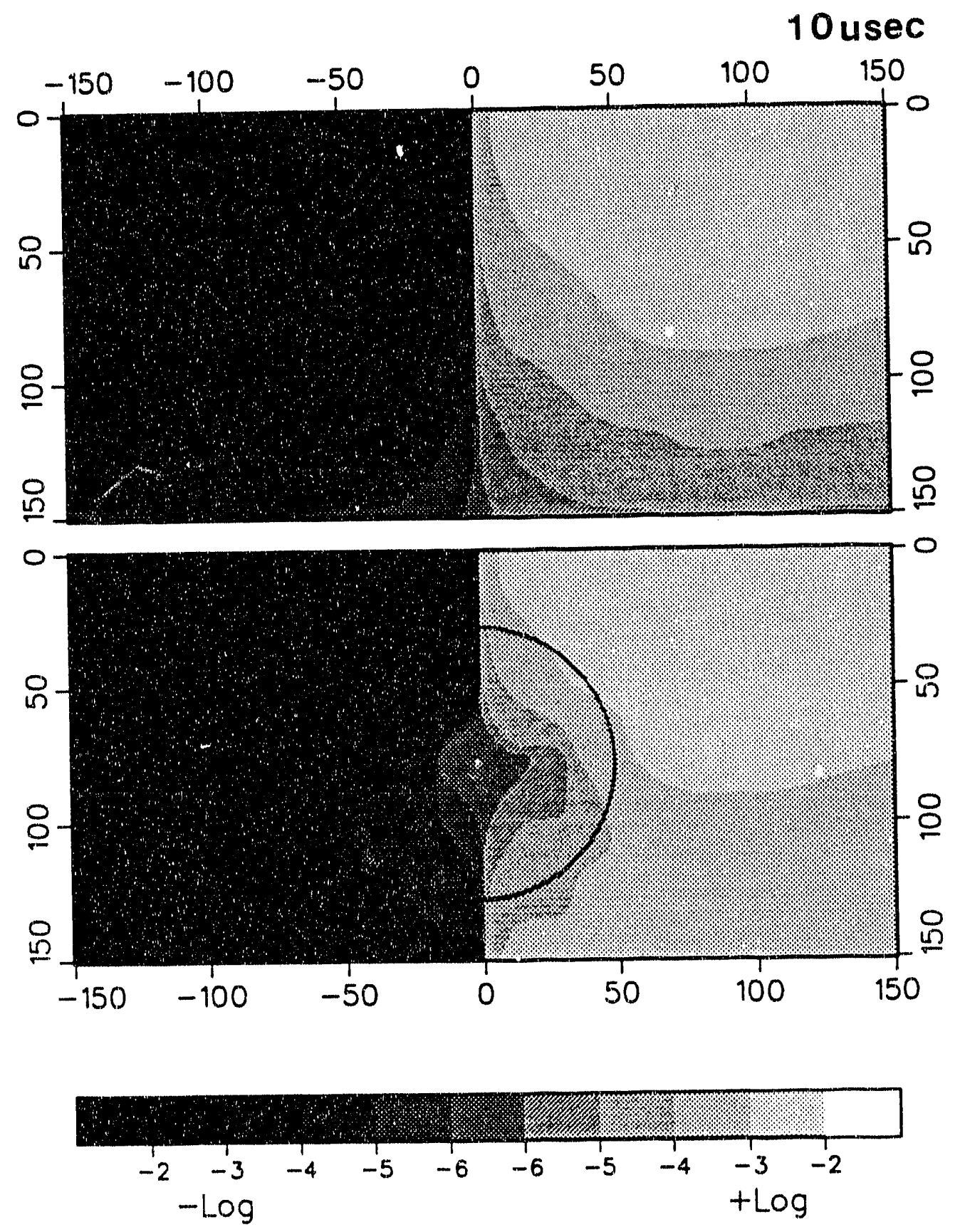

Figure 4-13 (b) Snapshots of half space and with sphere model at $t=10$ usec (Ey). 


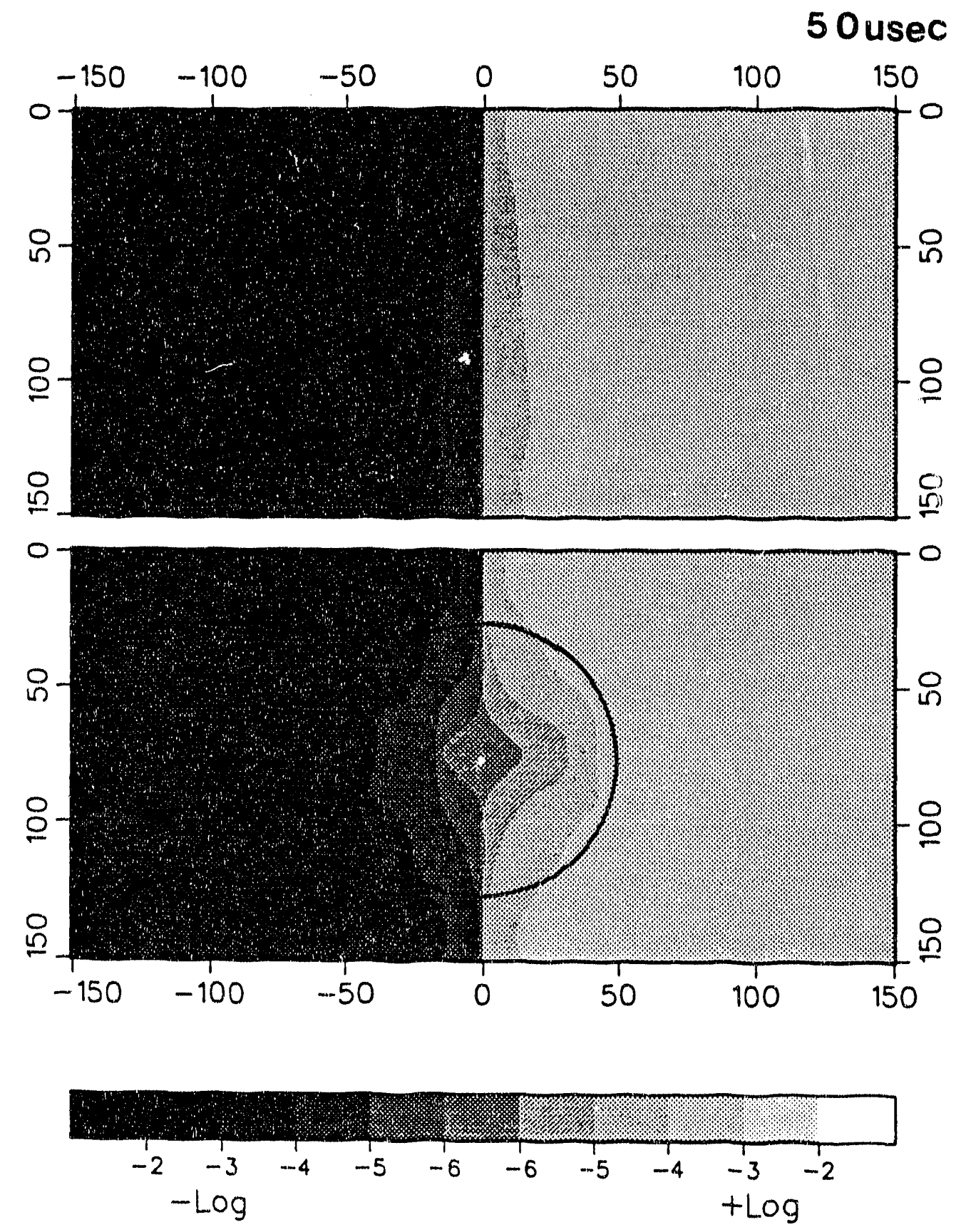

Figure 4-13 (c) Snapshots of half space and with sphere model at $t=50$ usec (Ey). 


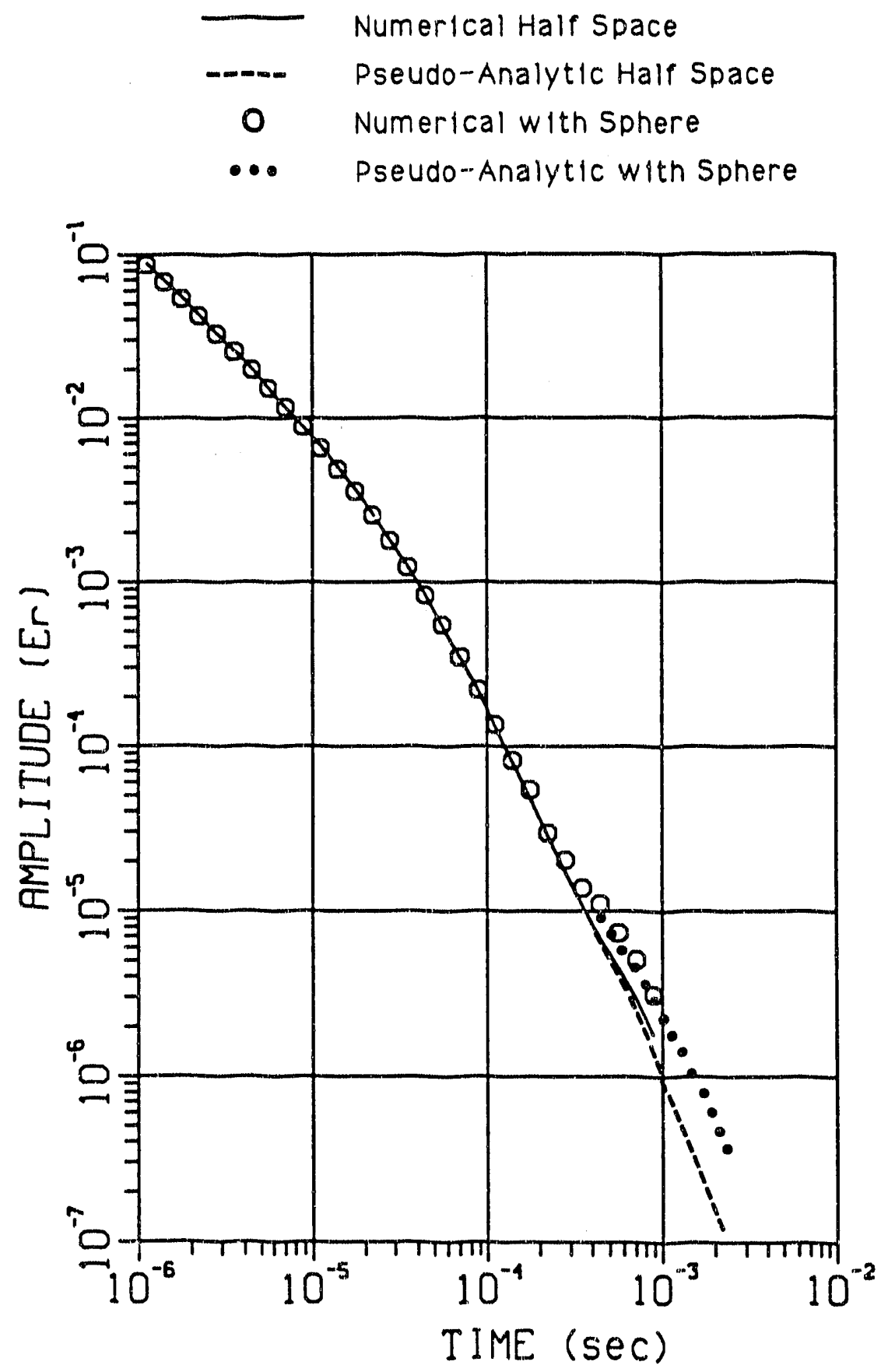

Figure 4-14 Comparisons with the sphere solution (Lee, 1976). The pseudo-analytic: sphere solution is evaluated only in late times. 


\section{Discussion, Useful Derivations, and Sum- mary}

The t-k algorithm has two main limitations for geophysical modelling. First, the resistivity contrast has to be small (less than 10) whereas many geophysical contrasts are expected to be greater than 10 . Next, as with any other explict time-stepping algorithm, when the source is close to an inhomogeneity or in a resistive medium, the starting time for the calculations has to be very small and the total number of calculations has to be increased.

As shown in the previous theoretical derivation, there is no fundamental reason that the resistivity contrast has to be small. Since the potentials $f(\vec{k}, t)$ and $g(\vec{k}, t)$ are band limited $\left(f\left(\vec{k}>\overrightarrow{k_{n}}, t\right), g\left(\vec{k}>\overrightarrow{k_{n}}, t\right) \equiv 0\right)$, from the definition of the sampling theorem (Bracewell, 1978), the electric current densities $J_{x, y, z}(\vec{r}, t)$ can be evaluated analytically at regularly spaced nodes by the fast Fourier transform. Since the resistivity $\rho(\vec{r})$ is defined as piecewise continuous, the electic fields $E_{x, y, z}(\vec{r}, t)$ are also defined analytically at each node $\left(E_{x, y, z}(\vec{r}, t)=J_{x, y, z}(\vec{r}, t) \rho(\vec{r})\right)$. But sharp changes in resistivity distribution make this band extremely wide (Figure 5.1), and the finite numerical accuracy of the fast Fourier transform creates numerical noise that can be amplified after multiplication by large resistivities. Figure 5.2 shows the analytic and numerical results of two symmetric Fourier transform pairs.

Most 3-D resistivity distributions can be approximated by 1 - or 2-D models at early times (Figure 5.3). For these cases, the amount of calculation for this alorithm is greatly reduced and the disadvantages can be treated by 
the following approach: The direct convolution in the wavenumber domain of $J_{x, y, z}(\vec{k}, t)$ and $\rho(\vec{k})$ for $E_{x, y, z}(\vec{k}, t)$ can prevent the numerical noise and aliasing effects possibly created by the fast Fourier transform. The implicit formulation of time-stepping can make this algorithm unconditionally stable and can be started at $t=0^{+}$when the sources are located on the inhomogeneous boundary. Finally, the harmonic, linear, and invariant characteristics in the wavenumber domain suggest that a useful imaging algorithm could be derived from this formulation.

\subsection{Direct Convolution}

An alternative approach to evaluate $E_{x, y, z}$ from $J_{x, y, z}$ is by a direct convolution in the wavenumber domain.

$$
\begin{gathered}
E_{x, y, z}(\vec{k}, t)=J_{x, y, z}(\vec{k}, t) \otimes \rho(\vec{k}) \\
E_{x, y, z}(\vec{k}, t)=\int J_{x, y, z}\left(\overrightarrow{k^{\prime}}, t\right) \rho\left(\vec{k}-\overrightarrow{k^{\prime}}\right) d \overrightarrow{k^{\prime}} .
\end{gathered}
$$

In this approach, $f(\vec{k}, t)$ and $g(\vec{k}, t)$ need not be regularly spaced. They can be closely spaced at the lower wavenumbers where the amplitudes are large and sparsely spaced at the higher wavenumbers where the amplitudes are small. This approach requires $n^{2}$ additions and multiplications for $n$ nodes rather than $n \log _{2} n$ calculation for the fast Fourier transform. But this calculation is very manageable for 1 - or $2-\mathrm{D}$ models and can prevent possible numerical noise that can be generated by the fast Fourier transform calculations. 

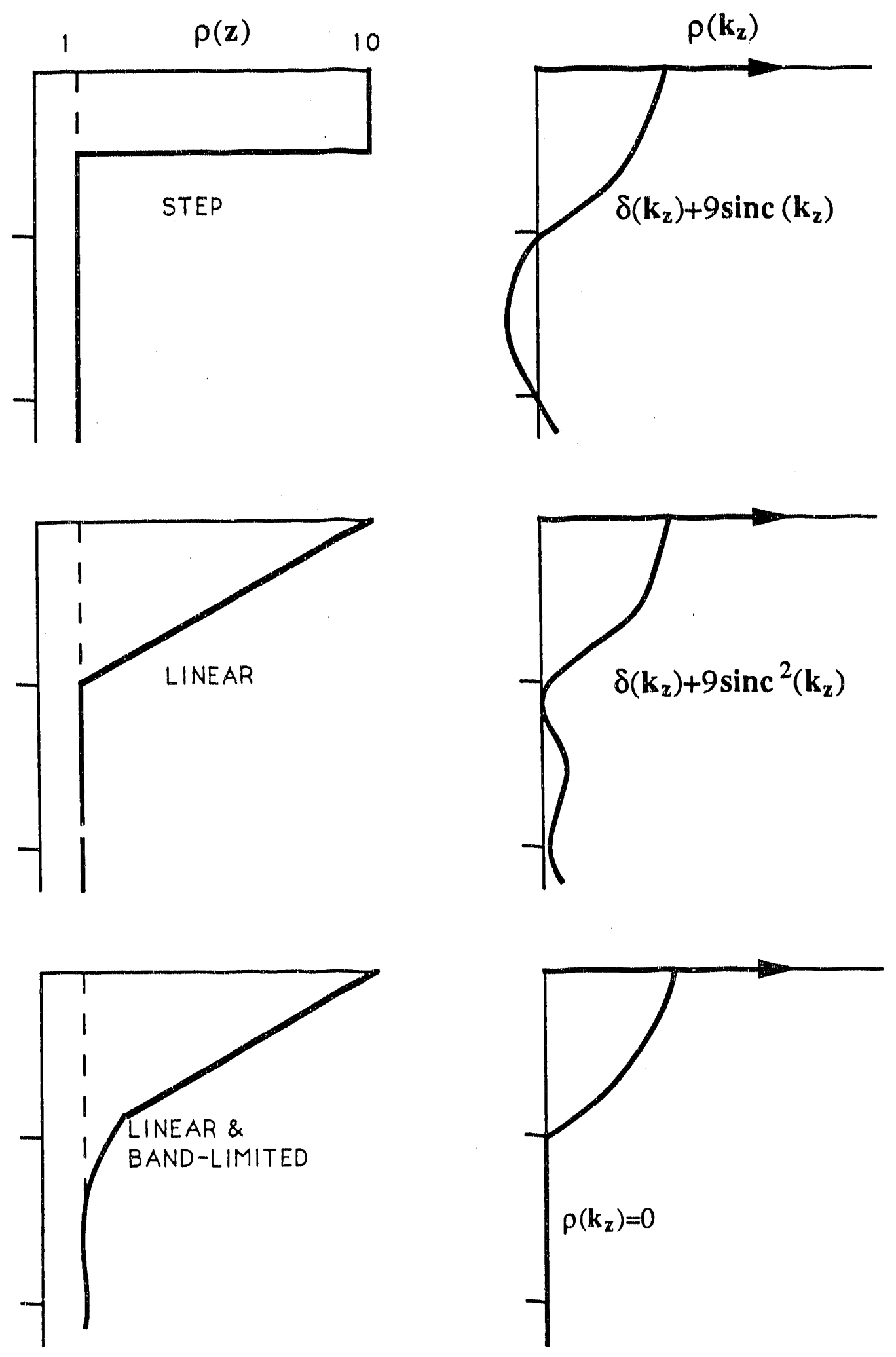

Figure 5-1 Examples of Fourier transform pair $(z<->k z)$. 

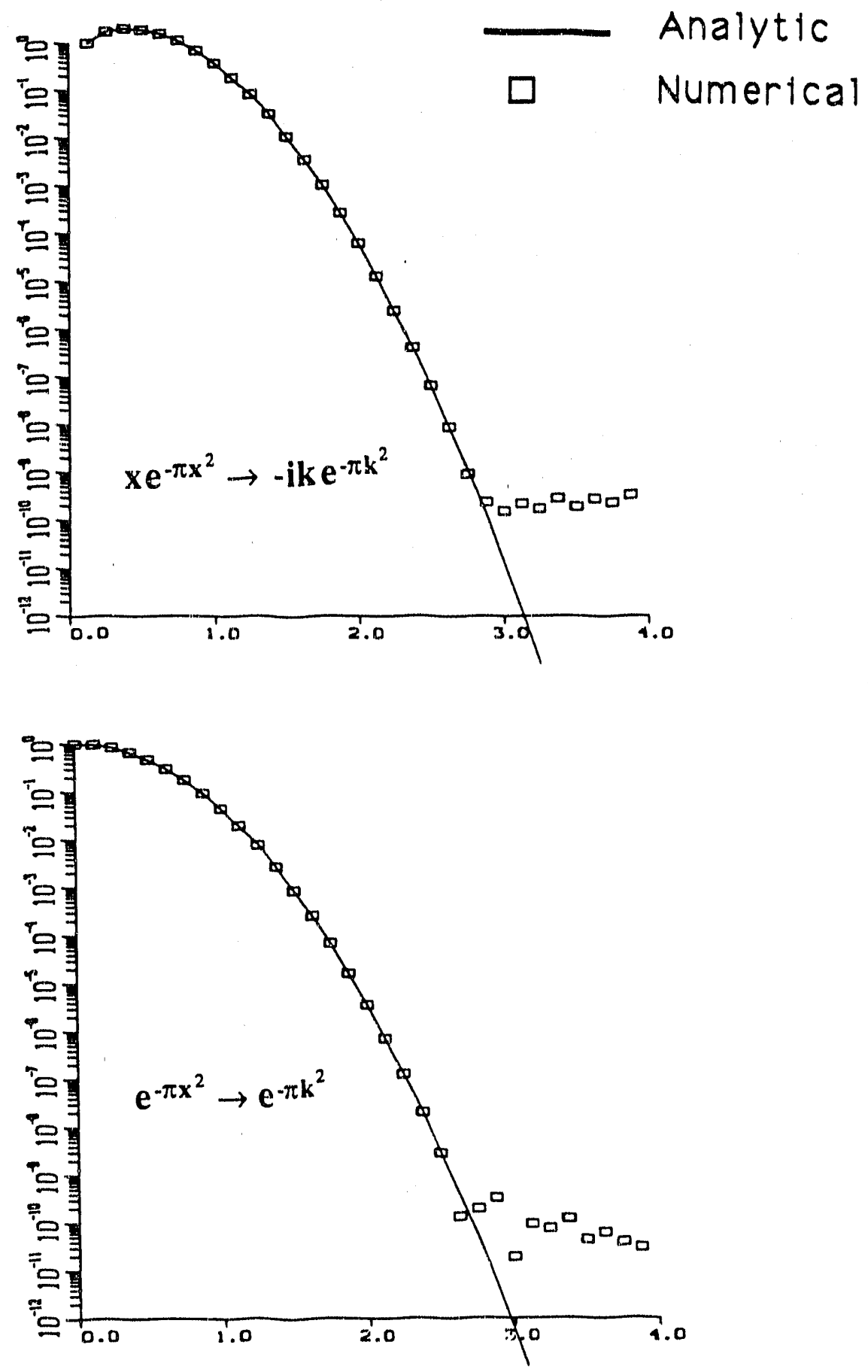

Figure 5-2 Results of double-precision ( 64 bit) floating-point FFT ( $N=32)$. The numerical precision range is $9 \sim 10$ decimal digits as shown in these figures. 

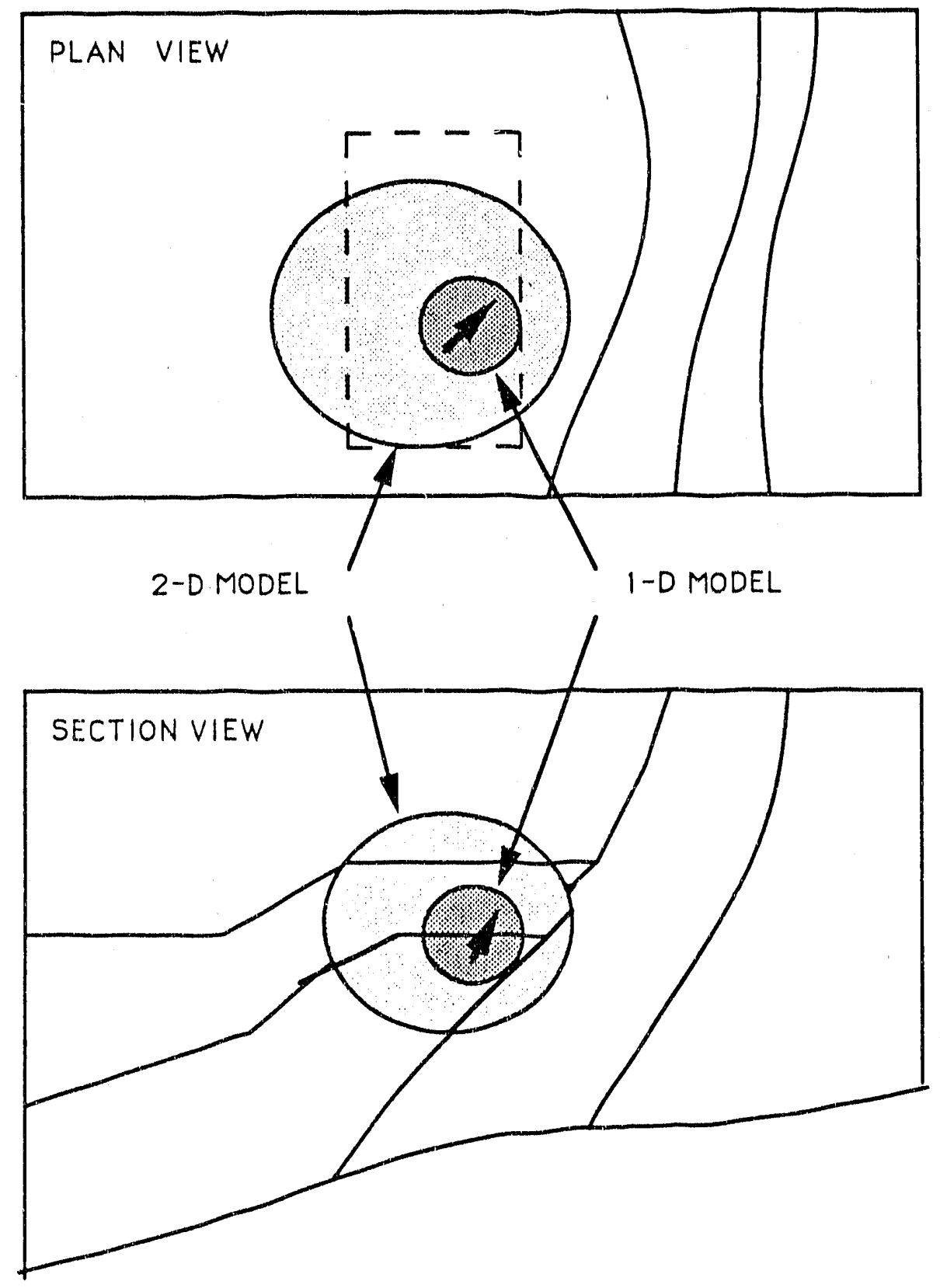

Figure 5-3 Schematic diagram of a realistic 3-D model and the area where one can use a 1D or a $2-D$ solution at early times. 
In the 1-D case, equation (2.13) to (2.17) can be further simplified as follows: by symmetry, let $k_{y}=0$,

For a vertical magnetic dipole,

$$
\mu_{0} \frac{\partial f\left(k_{x}, k_{z}, t\right)}{\partial t}=-\left(k_{x}^{2}+k_{z}^{2}\right)\left[f\left(k_{x}, k_{z}, t\right) \otimes \rho\left(k_{z}\right)\right] .
$$

For a vertical electric dipole,

$$
\mu_{0} \frac{\partial g\left(k_{x}, k_{z}, t\right)}{\partial t}=-\left(k_{x}^{2}+k_{z}^{2}\right)\left\{g\left(k_{x}, k_{z}, t\right) \otimes \rho\left(k_{z}\right)\right]
$$

For the 2-D case, these $f$ and $g$ are generally coupled, but only 2-D convolutions are required for $E_{x, y, z}(\vec{k}, t)$.

$$
E_{x, y, z}\left(k_{x}, k_{y}, k_{z}, t\right)=\iint J_{x, y, z}\left(k_{x}^{\prime}, k_{y}, k_{x}^{\prime}, t\right) \rho\left(k_{x}-k_{x}^{\prime}, k_{z}-k_{z}^{\prime}\right) d k_{x}^{\prime} d k_{z}^{\prime} .
$$

\subsection{Implicit Formula}

Using direct convolution in the wavenumber domain, this algorithm can be formulated into a fully implicit formula. Since the implicit method involves matrix inversion, it is not suitable for 3-D or large 2-D models. But it can be used for a large contrast 1-D resistivity model where numerical instability is suspected or where the sources are located on the inhomogeneous boundary so that the only possible initial time is $t=0^{+}$.

Let

$$
f\left(k_{x}, k_{z}\right)=\left|f_{-n}, f_{-n+1} \cdots f_{n-1}, f_{n}\right|
$$

and

$$
\rho\left(k_{z}\right)=\left|\rho_{-n}, \rho_{-n+1} \cdots \rho_{n-1}, \rho_{n}\right|,
$$


then equation (5.2) or (5.3) becomes

$$
\mu_{0} \frac{\partial}{\partial t}\left[\begin{array}{c}
f_{-n} \\
f_{-n+1} \\
\vdots \\
f_{n-1} \\
f_{n}
\end{array}\right]=-\left(k_{x}^{2}+k_{z}^{2}\right)\left[\begin{array}{ccccc}
\rho_{-n} & 0 & \cdots & 0 & 0 \\
\rho_{-n+1} & \rho_{-n} & \cdots & 0 & 0 \\
. & . & \cdots & \cdot & \cdot \\
0 & 0 & \rho_{n} & \rho_{n-1} \\
0 & 0 & 0 & \rho_{n}
\end{array}\right]\left[\begin{array}{c}
f_{-n} \\
f_{-n+1} \\
\vdots \\
f_{n-1} \\
f_{n}
\end{array}\right]
$$

Applying backward time differencing in equation (5.5),

$$
\left[f^{t+\delta t}\right]-\left[f^{t}\right]=-\frac{\delta t}{\mu_{0}}\left(k_{x}^{2}+k y^{2}\right)[\rho]\left[f^{t+\delta t}\right] .
$$

or

$$
\left[f^{t+\delta t}\right]=[A]^{-1}\left[f^{t}\right]
$$

where $[A]=\left(1+\frac{\delta t}{\mu_{0}}\left(k_{x}^{2}+k_{z}^{2}\right)[\rho]\right)$. The matrix $[A]$ is positive definite and this formulation is unconditionally stable (Mitchell and Griffiths, 1980).

\subsection{Time-Domain Imaging}

In seismic wave propagation, various imaging methods have been developed and successfully implemented, such as multisource holography and diffraction tomography (Devaney, 1984, 1985; Wu and Toksoz, 1987) and migration methods (Claerbout,1976; Gazdag, 1978). The basic underlying assumptions are: the source can be decomposed into plane waves, the targets are far away from both source and receiver, and the contrast of target parameters are small. But none of these assumptions appears to be acceptable in the practical electromagnetic diffusion process where the contrast of target parameters 
is usually greater than 10 , and exponentially decaying fields in both time and distance prohibits the observation when the targets are far away from the source or receiver.

From Figure (5.4), in the absence of $\sigma_{s}(\vec{r})$, Maxwell's equations becomes,

$$
\begin{gathered}
\nabla \times E_{p}(\vec{r}, t)=-\mu \frac{\partial H_{p}(\vec{r}, t)}{\partial t}, \\
\nabla \times H_{p}(\vec{r}, t)=\sigma_{p}(\vec{r}) E_{p}(\vec{r}, t)+J_{s r c}(\vec{r}, t) .
\end{gathered}
$$

In the presence of $\sigma_{s}(\vec{r})$,

$$
\nabla \times\left[E_{p}(\vec{r}, t)+E_{s}(\vec{r}, t)\right]=-\mu \frac{\left[\partial H_{p}(\vec{r}, t)+\partial H_{s}(\vec{r}, t)\right]}{\partial t},
$$

$$
\nabla \times\left[H_{p}(\vec{r}, t)+H_{s}(\vec{r}, t)\right]=\left(\sigma_{p}(\vec{r})+\sigma_{s}(\vec{r})\right)\left[E_{p}(\vec{r}, t)+E_{s}(\vec{r}, t)\right]+J_{s r c}(\vec{r}, t) .
$$

From equations (5.10) - (5.8) and (5.11) - (5.9),

$$
\begin{gathered}
\nabla \times E_{s}(\vec{r}, t)=-\mu \frac{\partial H_{s}(\vec{r}, t)}{\partial t}, \\
\nabla \times H_{s}(\vec{r}, t)=\left(\sigma_{p}(\vec{r})+\sigma_{s}(\vec{r})\right) E_{s}(\vec{r}, t)+\sigma_{s}(\vec{r}) E_{p}(\vec{r}, t) .
\end{gathered}
$$

Let $\rho(\vec{r})=\left(\sigma_{p}(\vec{r})+\sigma_{s}(\vec{r})\right)^{-1}$ then equation (5.13) becomes,

$$
\rho(\vec{r}) \nabla \times H_{s}(\vec{r}, t)=E_{s}(\vec{r}, t)+\rho(\vec{r}) \sigma_{s}(\vec{r}) E_{p}(\vec{r}, t) .
$$

Substituting equation (5.12) to the $\nabla \times$ equation (5.14),

$$
\nabla \times \rho(\vec{r}) \nabla \times H_{s}(\vec{r}, t)+\mu \frac{\partial H_{s}(\vec{r}, t)}{\partial t}=\nabla \times \rho(\vec{r}) \sigma_{s}(\vec{r}) E_{p}(\vec{r}, t) .
$$

Defining

$$
O(\vec{r})=\rho(\vec{r}) \sigma_{s}(\vec{r})=\frac{\sigma_{s}}{\sigma_{p}+\sigma_{s}}
$$



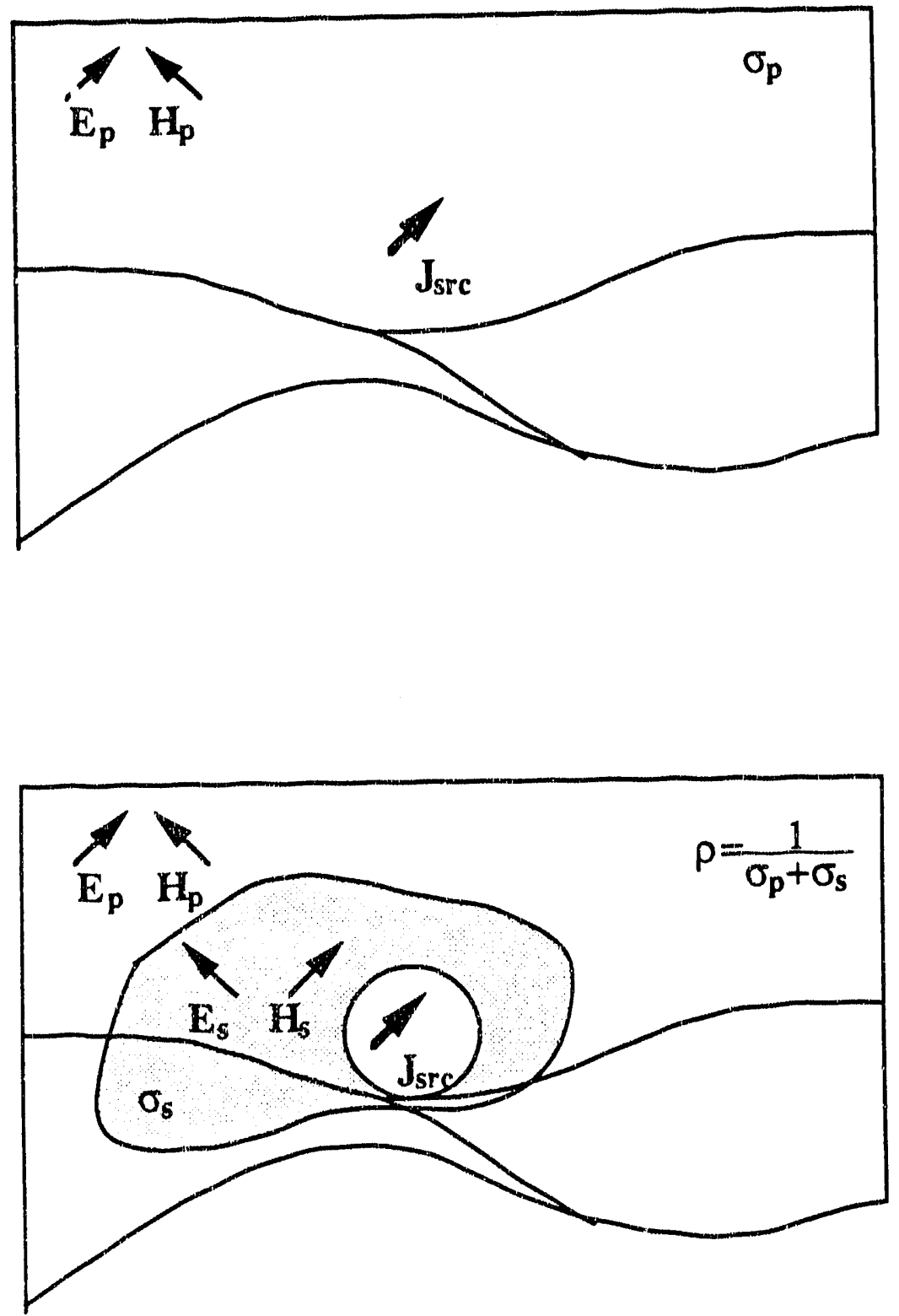

Figure 5-4 Prisin:! and sccondary conductivity distributions. Both distributions can be

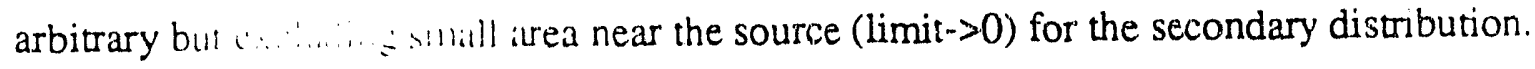


then

$$
-1<O(\vec{r})<1 \text { for }-\infty<\sigma_{s}<\infty
$$

Assuming $O(\vec{r})$ is smoothly varying,

$$
\begin{aligned}
\nabla \times O(\vec{r}) E_{p}(\vec{r}, t) & =\nabla O(\vec{r}) \times E_{p}(\vec{r}, t)+O(\vec{r}) \nabla \times E_{p}(\vec{r}, t) \\
& \approx O(\vec{r}) \nabla \times E_{p}(\vec{r}, t) \\
& =-\mu O(\vec{r}) \frac{\partial H_{p}(\vec{r}, t)}{\partial t},
\end{aligned}
$$

Equation (5.15) becomes,

$$
\nabla \times \rho(\vec{r}) \nabla \times H_{s}(\vec{r}, t)+\mu \frac{\partial H_{s}(\vec{r}, t)}{\partial t}=-\mu O(\vec{r}) \frac{\partial H_{p}(\vec{r}, t)}{\partial t} .
$$

The general solution for equation (5.17) is

$$
H_{s}(\vec{r}, t)=\int_{0}^{t} \int_{v^{\prime}}-\mu O\left(\overrightarrow{r^{\prime}}\right) \frac{\partial H_{p}\left(\overrightarrow{r^{\prime}}, t^{\prime}\right)}{\partial t} H_{r}\left(\vec{r}-\overrightarrow{r^{\prime}}, t\right) d r^{\prime} d t^{\prime}
$$

where $H_{r}(\vec{r}, t)$ is the Green's dyadic for the unknown resistivity distribution $\rho(\vec{r})$.

Excluding the small area surrounding the source,

$$
O\left(\overrightarrow{r^{\prime}}\right) H_{p}\left(\overrightarrow{r^{\prime}}, t=0\right)=0
$$

Equation (5.18) becomes

$$
H_{s}(\vec{r}, t)=\int_{v^{\prime}}-\mu O\left(\overrightarrow{r^{\prime}}\right) H_{p}\left(\overrightarrow{r^{\prime}}, t\right) H_{r}\left(\vec{r}-\overrightarrow{r^{\prime}}, t\right) d r^{\prime} .
$$

In the wavenumber domain, the convolution of $O$ and $H_{P}$ becomes inulitplication and the multiplication of $H_{r}$ becomes convolution to $O$ and $H_{p}$,

$$
H_{s}(\vec{r}, t)=-\mu \int\left[\int O(\vec{k}) H_{P}\left(\overrightarrow{k^{\prime}}-\vec{k}, t\right) d \vec{k}\right] H_{r}\left(\overrightarrow{k^{\prime}}, t\right) \exp \left(-j \overrightarrow{k^{\prime}} \cdot \vec{r}\right) d \overrightarrow{k^{\prime}}
$$




$$
=-\mu \int O(\vec{k})\left[\int H_{P}\left(\overrightarrow{k^{\prime}}-\vec{k}, t\right) H_{r}\left(\overrightarrow{k^{\prime}}, t\right) \exp \left(-j \overrightarrow{k^{\prime}} \cdot \vec{r}\right) d \overrightarrow{k^{\prime}}\right] d \vec{k}
$$

Let $\overrightarrow{k^{\prime}}-\vec{k}=\vec{k}$ then,

$H_{s}(\vec{r}, t)=-\mu \int O(\vec{k})\left[\int H_{P}(\vec{k}, t) H_{r}(\vec{k}+\vec{k}, t) \exp (-j \vec{k} \cdot \vec{r}) d \vec{k} \vec{k}\right] \exp (-j \vec{k} \cdot \vec{r}) d \vec{k}$

Let

$$
R(\vec{k}, t)=\int H_{P}(\vec{k}, t) \exp (-j \vec{k} \cdot \vec{r}) H_{r}(\vec{k}+\vec{k}, t) d \vec{k}
$$

then equation (5.21) becomes

$$
\begin{aligned}
H_{s}(\vec{r}, t) & =\int O(\vec{k}) R(\vec{k}, t) \exp (-j \vec{k} \cdot \vec{r}) d \vec{k} \\
& =\int_{v^{\prime}} O\left(\overrightarrow{r^{\prime}}\right) R\left(\vec{r}-\overrightarrow{r^{\prime}}, t\right) d \overrightarrow{r^{\prime}} .
\end{aligned}
$$

The governing equation (equation 5.10 and 5.11 ) has been transformed to the superposition of the secondary impulse source distribution $\left(O\left(\overrightarrow{r^{\prime}}\right)\right)$ with the corresponding response function $R(\vec{r}, t)$ that is the cross correlation of the primary field and Green's function in the wavenumber domain.

These derivations are the mathematical formulation of the fundamental physical concept that the response from a linear system is the correlation of a response function $R$ and an object $O$. Comparing to the formulation by Zhou (1989) which directly follows the seismic concepts, the input parameters are magnetic fields which are easily measurable in the subsurface geophysical explorations, the object function is bounded $(|O(\vec{r})| \leq 1)$ that can make nonunique inverse or imaging algorithm more stable, and the primary medium $\sigma_{p}$ need not to be homogeneous or layered space since the response function $R$ can be evaluated from numerical solutions. 


\subsection{Summary}

The $t-k$ algorithm fills the need for a time domain computation scheme for geophysical applications of electromagnetics. It is flexible so that the model response for various source configurations can be obtained by a simple iteration of initial conditions. It may be used to compute the system response over three decades in time in a $64 \times 64 \times 32$ node model. The only currently available algorithm for modelling a general 3-D structure without the inversion of matrices is the explicit finite difference scheme which is best suited for modern parallel computers. Its principal advantage is that only a few neighboring nodes are required in the calculations to update each node, nevertheless the number of nodes needed for any reasonable 3-D model quickly eliminates this advantage. For example, Oristaglio and Hohmann (1984) used $195 \times 79$ nodes in the $\mathrm{x}$ and $\mathrm{z}$ directions for a 2-D line source problem. The computation required 8.5 hours of CPU time on a VAX 11/780 computer. Computationally expanding this algorithm to a 3-D case with 100 nodes in the $y$ direction will require at least $1700 \mathrm{CPU}$ hours on a VAX 11/780 because the coupled nature of TE and TM modes in 3-D requires at least two hundred times the computation needed in 2-D. This is equivalent to about $5 \mathrm{CPU}$ hours on a Cray-2 with a parallel processor. As shown previously context, the $\mathrm{t}-\mathrm{k}$ algorithm only requires one CPU hour on a Cray-2 with one megaword storage.

Another novel feature of the $\mathrm{t}-\mathrm{k}$ algorithm is that one can estimate the accuracy of numerical results better than for other algorithms. Usually numerical solutions show the accuracy as a mathematical expression which is a function of the grid-spacing and time-step

$\left(\mathrm{O}\left(\mathrm{dx}^{2}\right)\right.$ and $\mathrm{O}\left(\mathrm{dt}^{2}\right)$ etc. $)$. The accuracy of the $\mathrm{t}-\mathrm{k}$ results however, is clearly defined by the truncation of the potentials and the conductivity structure in the wavenumber domain. 


\section{Appendix (Loop Source)}

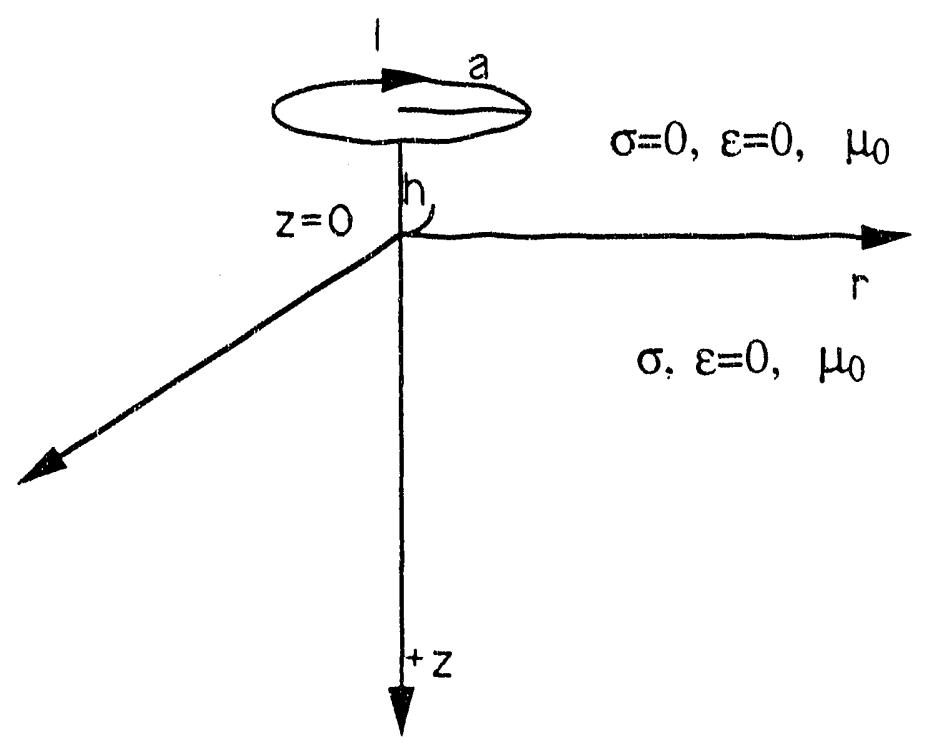

Tangential electric field $E_{\theta}$ inside the earth by a horizontal loop located on or above a homogeneous half space is given by (Hohmann, 1985),

$$
E_{\theta}(r, z, \omega)=-i \omega \mu_{0} I a \int_{0}^{\infty} e^{-\lambda h} \frac{J_{1}(\lambda a) J_{1}(\lambda r)}{\lambda+\sqrt{s^{\prime}}} e^{-\sqrt{s^{\prime}} z} d \lambda
$$

where

$$
\begin{gathered}
\omega=\text { angular frequency }[H z] \\
\lambda=\sqrt{k_{x}^{2}+k_{y}^{2}}
\end{gathered}
$$




$$
\begin{gathered}
s^{\prime}=\sqrt{\lambda^{2}+i \mu_{0} \sigma \omega} \\
I=\text { current }[A] \\
a=\text { radius of the loop }[\mathrm{m}] \\
h=\text { height of the loop }[\mathrm{m}] \\
J_{1}=1^{\text {st }} \text { order Bessel function. }
\end{gathered}
$$

Using a Bessel function relation,

$$
J_{1}(\lambda r)=-\frac{1}{\lambda} \frac{\partial}{\partial r} J_{0}(\lambda r)
$$

and

$$
\begin{gathered}
J_{\theta}=\sigma E_{\theta}, \\
J(r, z, s)=-s \sigma \mu_{0} I a \frac{\partial}{\partial r} \int_{0}^{\infty} e^{-\lambda h} \frac{J_{1}(\lambda a) J_{0}(\lambda r)}{\lambda+\sqrt{s^{\prime}}} e^{-\sqrt{s^{\prime} z}} d \lambda
\end{gathered}
$$

where $s=-i \omega$. From equation (2.13),

$$
\begin{gathered}
J_{x}\left(k_{x}, k_{y}, k_{z}\right)=k_{y} f\left(k_{x}, k_{y}, k_{z}\right), \\
J_{\theta}(\lambda, z)=-i \frac{\partial}{\partial r} f(\lambda, z) .
\end{gathered}
$$

The step current response $f(\lambda, z)$ becomes,

$$
f(\lambda, z, s)=i \sigma \mu_{0} I a \frac{1}{\lambda} e^{-\lambda h} \frac{J_{1}(\lambda a)}{\lambda+\sqrt{s^{\prime}}} e^{-\sqrt{s^{\prime}} z} .
$$

Transforming $z$ to $k_{z}$,

$$
f\left(\lambda, k_{z}, s\right)=M \sigma \mu_{0}\left[\frac{1}{\lambda+\sqrt{s^{\prime}}} \frac{\sqrt{s^{\prime}}}{k_{z}^{2}+s^{\prime}}\right]
$$


or

$$
f\left(\lambda, k_{z}, s\right)=M \sigma \mu_{0}\left[\frac{1}{\sqrt{s^{\prime}}\left(\lambda+\sqrt{s^{\prime}}\right)}\left(1-\frac{k_{z}^{2}}{k_{z}^{2}+s^{\prime}}\right)\right]
$$

where $M=2 i \operatorname{Ia} J_{1}(\lambda a) e^{-\lambda h} / \lambda$.

Using the following Lpalace transforms (Abrarmowitz and Stegun, 1964),

$$
\begin{gathered}
\frac{1}{\sqrt{s^{\prime}}\left(\sqrt{s^{\prime}}+a\right)} \rightarrow e^{a^{2} t} \operatorname{erf} c(a \sqrt{t}), \\
\frac{1}{s+a} \rightarrow e^{-a t}, \\
f(s) g(s) \rightarrow \int_{0}^{t} F(u) G(t-u) d u, \\
f(c s+b) \rightarrow(1 / c) e^{(-b / c) t} F(t / c), \\
f\left(\lambda, k_{z}, t\right)=M\left[\operatorname{erf} c\left(\lambda \sqrt{t^{\prime}}\right)-k_{z}^{2} \int_{0}^{t^{\prime}} \operatorname{erfc}(\lambda \sqrt{u}) e^{-\left(\lambda^{2}+k_{z}^{2}\right)\left(t^{\prime}-u\right)} d u\right], \\
=M\left[\operatorname{erfc}\left(\lambda \sqrt{t^{\prime}}\right)-k_{z}^{2} e^{-\left(\lambda^{2}+k_{z}^{2}\right) t^{\prime}} \int_{0}^{t^{\prime}} \operatorname{erfc}(\lambda \sqrt{u}) e^{\left(\lambda^{2}+k_{z}^{2}\right) u} d u\right]
\end{gathered}
$$

where $t^{\prime}=t /\left(\sigma \mu_{0}\right)$. Integrating by part,

$$
\begin{gathered}
\int_{0}^{t^{\prime}} \operatorname{erfc}(\lambda \sqrt{u}) e^{\left(\lambda^{2}+k_{z}^{2}\right) u} d u= \\
{\left[\operatorname{erfc}(\lambda \sqrt{u}) \frac{e^{\left(\lambda^{2}+k_{z}^{2}\right) u}}{\lambda^{2}+k_{z}^{2}}\right]_{0}^{t^{\prime}}-\int_{0}^{k_{z} \sqrt{t^{\prime}}} \frac{\lambda}{k_{z}} \frac{-2}{\sqrt{\pi}} \frac{e^{u^{2}}}{\lambda^{2}+k_{z}^{2}} d u .}
\end{gathered}
$$

Finally,

$$
f\left(\lambda, k_{z}, t\right)=\frac{M}{\lambda^{2}+k_{z}^{2}}\left(\lambda^{2} \operatorname{erf} c\left(\lambda \sqrt{t^{\prime}}\right)+k_{z}^{2} e^{-\left(\lambda^{2}+k_{z}^{2}\right) t^{\prime}}-\frac{2}{\sqrt{\pi}} \lambda k_{z} e^{-\lambda^{2} t^{\prime}} F\left(k_{z} \sqrt{t^{\prime}}\right)\right)
$$

where $F(x)$ is Dawson's integral defined as,

$$
F(x)=e^{-x^{2}} \int_{0}^{x} e^{u^{2}} d u
$$




\section{REFERENCES}

Abramowitz, M., and Stegun, I. A., 1964, Handbook of mathematical functions: New York, Dover Pub. Inc.

Adhidjaja, J. I., Hohmann, G. W., and Oristaglio, M. L., 1985, Two-dimensional transient electromagnetic responses: Geophysics 50, 2849-2861.

Anderson, W. L., 1975, Improved digital filters for evaluating Fourier and Hankel transforms integrals: Nat. Tech. Inf. Serv. rep. Pb-242-800.

Bracewell, R. N., 1978, The Fourier transform and its applications: New York, Mc-Graw Hill Book Co.

Claerbout, J. F., 1976, Fundamentals of geophysical data processing: New York, McGraw Hill Book Co.

Coggon, J. H., 1971, Electromagnetic and electrical modeling by the finite element method: Geophysics, 36, 132-155.

Devaney, A. J., 1984, Geophysical diffraction tomography: IEEE Trans. Geosci. Remote Sensing, GE-22, 3-13.

130 .

1985, Variable density acoustic tomography: J. Acoust. Soc. Am., 78, 120-

Engquist, B., and Majda, A., 1977, Absorbing boundary conditions for the numerical simulation of wave: Math. Comp., 31, 629-651.

Frischknecht, F. C., 1987, Electromagnetic physical scale modeling: in Nabighian, M. N. Ed.: Electromagnetic methods in applied geophysics - theory, vol. 1.

Gazdag, J., 1978, Wave-equation migration with the phase-shift method: Geophysics, 43, 1342-1351.

Geophysics, 46, 854-864. 
Goldman, M. M., and Stoyer, C. H., 1983, Finite-difference calculations of the transient field of an axially symmetric earth for vertical magnetic dipole excitation: Geophysics, 48, 953-963.

Gupta, P. K., Bennett, L. A., and Raiche, A. P., 1987, Hybrid calculations of the threedimensional electromagnetic response of buried conductors: Geophysics, 52, 501-306.

Harrington, R. F., 1961, Time-harmonic electromagnetic fields, New York, McGraw-Hill Book Co.

Hermance, J. F., 1982, Refined finite-difference simulations using local integral forms: Application to telluric fields in two dimensions: Geophysics, 47, 825-831.

Hohmann, G. W., 1971, Electromagnetic scattering by conductors in the earth near a line source of current: Geophysics, 36, 101-131.

-........., G. W., 1975, Three-dimensional induced polarization and electromagnetic modeling: Geophysics, 40, 309-324.

Jones, F. W. and Price, A. T., 1969, The peturbation of an alternating field by a conductve anomalies: Geophys. J. R. astr. Soc., 20, 317-334.

Kaufman, A. A., and Keller, G. V., 1983, Frequency and transient soundings: New York, Elsevier.

Kosloff, D. D., and Baysal, E., 1982, Forward modeling by a Fourier method: Geophysics, 47, 1402-1412.

Kosloff, D. D., Reshef, M., and Loewenthal D., 1984, Elastic wave calculations by the Fourier method: Bull. of the Seis. Soc. of America, 74, 875-891.

Kunz, K. S., and Lee, K. M., 1978, A three-dimensional finite difference solution of the external response of an aircraft to a complex transient EM Environment: IEEE Trans. Elec. Cornp., EMC-20, 328-341. 
Kuo, J. T. and Cho, D. H., 1980, Transient time-doniain electromagnetics: Geophysics, $45,271-291$.

Lee, K. H., Pridmore, D. F., and Morrison, H. F., 1981, A hybrid three-dimensional electromagnetic modeling scheme: Geophysics, 46, 796-805.

Lee, T., 1975, Transient electromagnetic response of a sphere in a layered medium: Geophys. Prosp., 23, 492-512.

Lines, L. R. and Jones, F. W., 1973, The perturbation of alternating geomagnetic fields by three-dimemsional island structures: Geophys. J. Roy. Soc., 32, 133-154.

Mitchell, A. R., and Griffiths, D. F., 1980, The finite difference method in partial differential equations: New York, John Wiley and Sons.

Nabighian, M. N., 1979, Quasi-static transient response of a conducting half-space: An approximate representation: Geophysics, vol. 44, 1700-1705.

Newman, G. A., Hohmann, G. W., and Anderson, W. L., 1986, Transient electromagnetic response of a three-dimensional body in a layered earth, Geophysics, 51, 1608-1627.

Oppenheim, A. V., and Schafer, R. W., 1975, Digital signal processing: New Jersey, Prentice-Hall Inc.

Oristaglio, M. L., and Hohmann, G. W., 1984, Diffusion of electromagnetic fields into a two-dimensional earth: A finite-difference approach: Geophysics, 49, 870-894.

Pridmore, D. F., Hohmann, G. W., Ward, S. H., and Sill, W. R., 1981, An investigation of finite-element modeling for electrical and electromagnetic data in three dimensions: Geophysics, 46, 1009-1024.

Raiche, A. P., 1974, An integral equation approach to 3D modeling: Geophys. J. Roy. Astron. Soc., 36, 363-376. 
Reddy, I. K., Rankin, D., and Philips, R., J., 1977, Three-dimensional medeling in magnetotelluric and magnetic variational sounding: Geophys. J. Roy. Astron. Soc., 51, 313-325.

Richtmyer, R. D., and Morton, K. W., 1967, Difference methods for initial value problems: New York, John Wiley and Sons, 2nd ed.

San Filipo, W. A., Eaton, P. A., and Hohmann, G. W., 1985, Integral equation solution for the transient electromagnetic response of a three-dimensional body in a conductive halfspace: Geophysics, 50, 798-809.

Swift, Jr. C. M., 1971, Theoretical magnetotelluric and turan response from twodimensional inhomogeneity: Geophysics, 36, 38-52.

Tayior, C. D., Lam, D. H., and Shumpert, T. H., 1969, Electromagnetic pulse scattering in time-varying inhomogeneous media: IEEE Trans. Antennas Propagat. AP-17, 585-589.

Telford, W. M., Geldart, L. P., Sheriff, R. E., and Keys, D. A., 1976, Applied geophysics: Cambridge, Cambridge Univ. Press.

Ward, S. H., and Hohmann, G. W., 1988, Electromagnetic theory for geophysical applications: in Nabighian M. N., ed: Electromagnetic Msthods in Applied GeophysicsTheory, vol. 1.

Weaver, J. T. and Brewitt-Taylor, C. R., 1978, Improved boundary condition for the numerical solution of E-polarization problems in geomagnetic induction: Geophys. J. R. astr. Soc., 54, 309-317.

Weidelt, P., 1975, Electromagnetic induction in three-dimensional structures. J. Geophys., 41, 85-109.

Wu, R., and Toksoz, M. N., 1987, Diffraction tomography and multisource holography applied to seismic imaging: Geophysics, 52, 11-25.

Yee, K. S., 1966, Numerical solution of initial boundary value problems involving Maxwell's equations in isotropic media: IEEE Trans. Ant. Prop., AP-14, 302-309. 
Zhdanov, M. S., Golubev, N. G., Spichack, V. V.,and Varentsov, Iv. M., 1982, The construction of effective methods for electromagnetic modelling: Geophys. J. Roy. Astron. Soc., 68, 589-607.

Zhou, Q., 1989, Audio frequency electromagnetic numerical medeling and tomographic inversion for reservoir evaluation: Ph.D. Thesis, Univ. of California at Berkeley. 

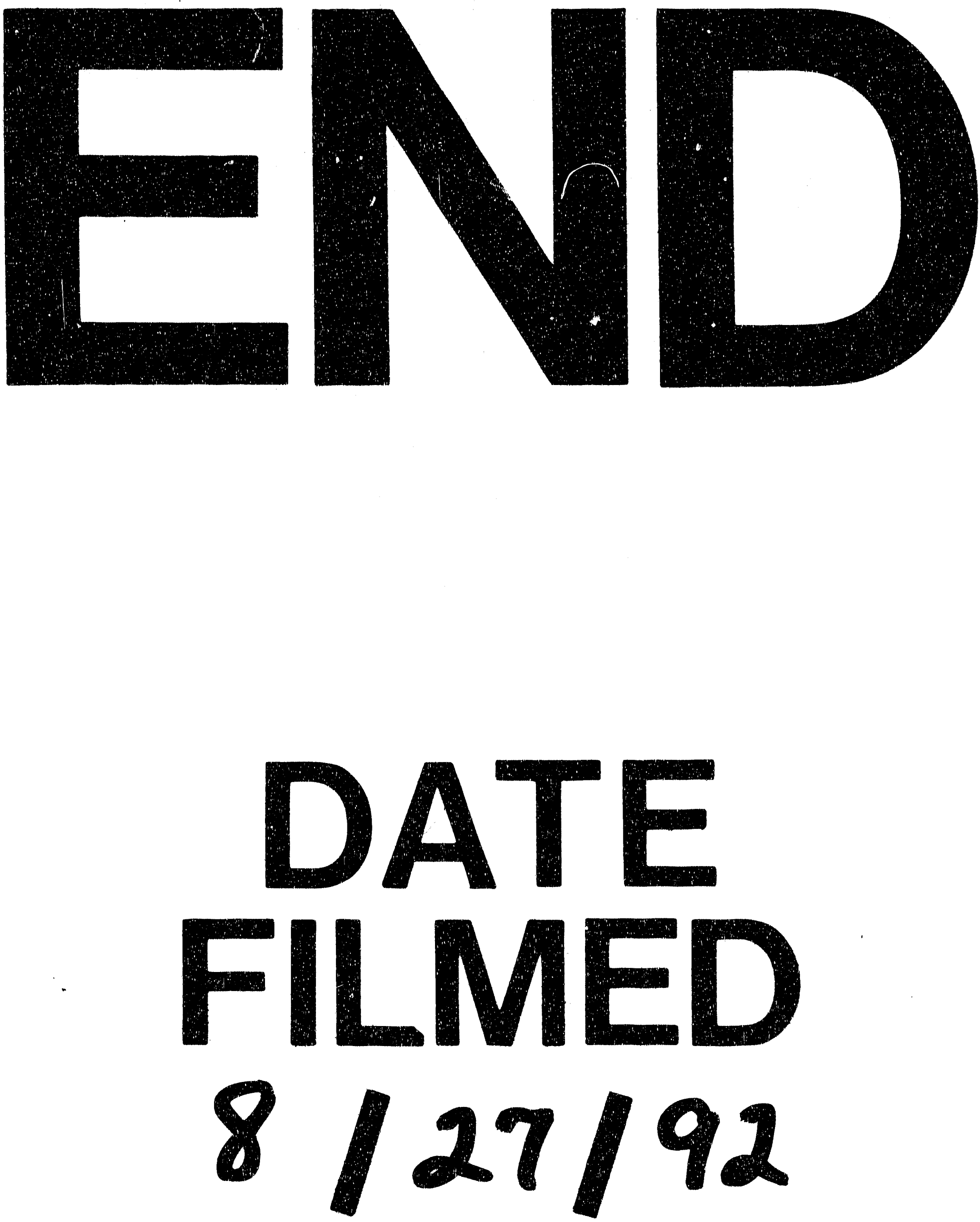
